\title{
Development of a phased-array ionospheric imaging system
}

by

Nicholas Bruce

B.Eng, University of Victoria, 2016

A thesis submitted in partial fulfillment of the requirements for the degree of Master of Applied Science

in the Department of Mechanical Engineering

(C) Nicholas Bruce, 2019

University of Victoria

All rights reserved. This thesis may not be reproduced in whole or in part, by photocopy or other means, without the permission of the author. 


\section{Supervisory Committee}

Development of a phased-array ionospheric imaging system

by

Nicholas Bruce

B.Eng, University of Victoria, 2016

\section{Supervisory Committee}

Supervisor:

Dr. Rodney Herring

Department of Mechanical Engineering

Co-supervisor:

Dr. Peter Driessen

Department of Electrical and Computer Engineering 


\title{
UNIVERSITY OF VICTORIA
}

\author{
Abstract \\ Faculty of Engineering \\ Department of Mechanical Engineering \\ Master of Applied Science \\ Development of a phased-array ionospheric imaging system \\ by Nicholas Bruce \\ A novel approach to ionospheric imaging with the purpose of weather/distaster \\ prediction and climate study is introduced. This feasibility study combines tradi- \\ tional material imaging techniques with high frequency (HF) radio via SDR (soft- \\ ware defined radio) systems in order to capture three-dimensional images of the \\ atmosphere. An experiment is devised and the necessary instrumentation built in \\ order to capture coherent images of the ionosphere. The experimental results show \\ these three-dimensional images as well as a novel approach to measuring iono- \\ spheric height. The novelty of the research comes from the use of a closely spaced \\ phased-array of radio antennas in conjunction with a post-correlation beamformer \\ repurposed from radio astronomy. Experiments were run at both the University of \\ Victoria and DRAO (Dominion Radio Astrophysical Observatory), the results which \\ led to a successful proposal for extending the research onto a larger array with sup-
} port from research groups in New Mexico. 


\section{Contents}

$\begin{array}{ll}\text { Supervisory Committee } & \text { ii }\end{array}$

$\begin{array}{ll}\text { Abstract } & \text { iii }\end{array}$

$\begin{array}{ll}\text { Contents } & \text { iv }\end{array}$

List of Figures

List of Tables viii

Acknowledgements $\quad$ ix

List of Abbreviations $\quad x$

1 Introduction 1

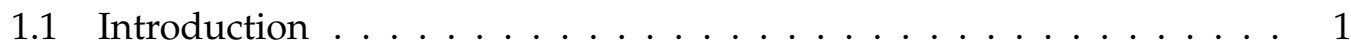

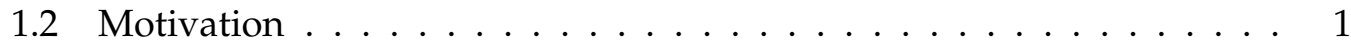

1.3 Contributions .................... 2

1.3.1 Development and running of an experiment at DRAO . . . . 2

1.3.2 Preliminary data analysis . . . . . . . . . . . . . 3

1.3.3 Miscellaneous . . . . . . . . . . . . . . . . . . . 3

1.3.4 Summary of contributions . . . . . . . . . . . . . 3

1.4 Structure of the thesis . . . . . . . . . . . . . . . . . . 3

2 Literature Review 4

2.1 Contextual vocabulary . . . . . . . . . . . . . . . 4

2.2 Ionospheric physics and chemistry . . . . . . . . . . . . . 4

2.3 Ionospheric variance . . . . . . . . . . . . . . . 6

2.4 Ionospheric disturbances . . . . . . . . . . . . . . . . . 7

2.4 Earthquakes . . . . . . . . . . . . . . . 8

2.4 .2 Weather and storms . . . . . . . . . . . . . . . . . 11

2.4 .3 Man-made disturbances . . . . . . . . . . . . . . . . . . . 11

2.5 Research efforts to image ionospheric disturbances . . . . . . . . . . . . 11

2.6 Concluding remarks . . . . . . . . . . . . . . 12

3 Methods 16

3.1 Motivation . . . . . . . . . . . . . . . . . . 16

3.2 Experimental preparation . . . . . . . . . . . . . . . 16

3.3 Experimental configuration $\ldots \ldots \ldots \ldots \ldots \ldots \ldots$

3.3 .1 Transmitter . . . . . . . . . . . . . . . 17

3.3 .2 Receiver . . . . . . . . . . . . . . . . . 17

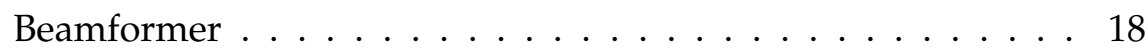

Receiver array . . . . . . . . . . . . . . . . 19

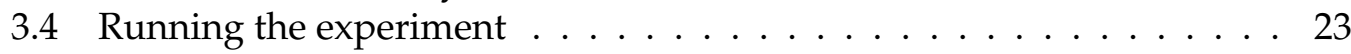


4 Results and Discussion $\quad 25$

4.1 Visualizing data during collection . . . . . . . . . . . . . 25

4.1.1 Radio frequency interference . . . . . . . . . . . . . 25

4.1.2 Spatial and frequency coherence . . . . . . . . . . . . 28

4.2 Visualizing data after collection . . . . . . . . . . . . . 28

4.2.1 Single frequency time domain . . . . . . . . . . 30

4.2 .2 Interpolating over missing data . . . . . . . . . . . . . 30

4.2.3 Considering average phase differences . . . . . . . . . 30

4.2.4 Mesh of ionospheric surface . . . . . . . . . . . . . . . 30

4.2 .5 Frequency domain analysis . . . . . . . . . . . . 33

4.2 .6 Virtual height . . . . . . . . . . . . . . . 33

5 Conclusion and future work $\quad 42$

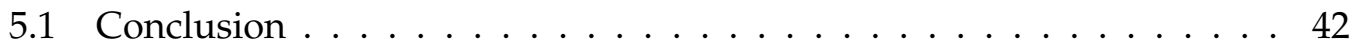

5.2 Future work . . . . . . . . . . . . . . . . . 42

Appendix A Setup and preparation for DRAO experiment 44

A.1 Experimental preparation . . . . . . . . . . . . . . . . 44

Appendix B Preliminary study at UVic $\quad 52$

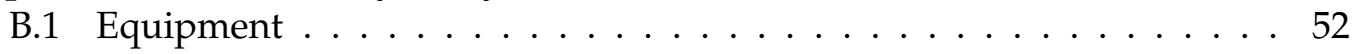

B.1.1 SDR system . . . . . . . . . . . . . . . 52

B.1.2 Power amplifier . . . . . . . . . . . . . . . 52

B.1.3 Antennas ........................ 53

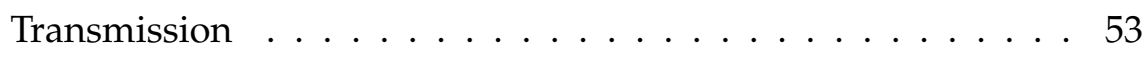

Reception ....................... 53

B.2 Experimental results . . . . . . . . . . . . . 53

B.2.1 Simulation . . . . . . . . . . . . . . . 53

Multipath channel model . . . . . . . . . . . . . . 54

Watterson ionospheric model . . . . . . . . . . . . 54

B.2.2 Expected values . . . . . . . . . . . . . . . . . 55

B.2.3 Long range two path . . . . . . . . . . . . . . . . 56

B.3 Discussion and interpretation . . . . . . . . . . . . . . 58

B.3.1 Skywave correlation . . . . . . . . . . . . . 58

B.3.2 Groundwave phase measurements . . . . . . . . . . . . . 58

B.4 Conclusion . . . . . . . . . . . . . . . . . . . . 59

$\begin{array}{lll}\text { Appendix C LWA proposed work } & 60\end{array}$

Appendix D Miscellaneous work done in support of a Masters of Applied

Science $\quad 61$

D.1 GNU Radio implementation of Flex radio . . . . . . . . . . . . . . . 61

D.1.1 Motivation . . . . . . . . . . . . . . . . 61

D.1.2 Components . . . . . . . . . . . . . . . . . . 61

GNU Radio . . . . . . . . . . . . . . . . . . . . . 6 61

FlexRadio $6700 \ldots \ldots$. . . . . . . . . . . . . 61

D.1.3 Implementation . . . . . . . . . . . . . . . . . . . . 62

D.1.4 Results . . . . . . . . . . . . . . . . . . . . 62

D.1.5 Existing issues . . . . . . . . . . . . . . . . . . . . 62

D.1.6 Release . . . . . . . . . . . . . . . . . . . . . 64

$\begin{array}{lr}\text { Bibliography } & 65\end{array}$ 


\section{List of Figures}

2.1 Ionospheric $\mathrm{E}$ and $\mathrm{F}$ regions showing that the F-region consists of F1 and $\mathrm{F} 2$ layers. . . . . . . . . . . . . . . . . . 4

2.2 Relative gas density, radiation intensity, and ionization of the atmosphere vs height [7]. . . . . . . . . . . . . . . . . . 5

2.3 Difference between actual refraction height and virtual reflection height. 6

2.4 Ionogram showing ionospheric height [8]. The two F2 curves are due to polarization of the radiowave by the ionosphere. . . . . . . 7

2.5 Historical solar activity [9] showing an 11-year solar cycle. . . . . . . . 7

2.6 Time delay results between the ground response (blue) and ionosphere response (red) to the 2015 earthquake in Nepal from two different locations, giving a measure of $10000 \mathrm{~km} / \mathrm{h}$ along the ground and $8000 \mathrm{~km} / \mathrm{hr}$ through the ionosphere [4]. . . . . . . . . . . .

2.7 (a) success rate and (b) probability score of precursors for earthquakes of $M>5.0$ and $M>6.0$ respectively [adapted from Chen, Liu, Tsai, et al., 2004 [2]]. . . . . . . . . . . . . . . . . . . . . . . 10

2.8 GPS stations used to image earthquake precursors prior to Chile's major 2015 earthquake. Red dots show GPS stations, white triangles in red dots show GPS/GLONASS stations, the green dot is the earthquake epicenter [21]. . . . . . . . . . . . . . . . . . 13

2.9 Electron density maps by height and time showing a wave travelling through the ionosphere before the 2015 Chilean earthquake struck [21]. 14

3.1 Experimental areas at DRAO . . . . . . . . . . . . . 20

3.2 Orientation in which the antennas were setup. . . . . . . . . . . 21

3.3 Block diagram of receiver system. . . . . . . . . . . . . . . . 22

3.4 Histogram of ADC (10-bit) channel voltages. Blue curves are ADC channels of active inputs while green curves are channels which are are not attached to anything. . . . . . . . . . . . . 22

3.5 Final array configurations of antennas, see Table 3.3 . . . . . . . . . . . 24

4.1 Average magnitude and phase of the ACM. White indicates no data or 0 as is the case for all of the diagonal elements of the phase (autocorrelation has no phase). Where the indices are the same, an autocorrelation was done, where they differ, a cross-correlation was done. The index shows the antenna element (see Figure 3.5). Each plot represents a $0.4 \mathrm{~s}$ snapshot of the data. . . . . . . . . . . . . . . 26

4.2 Array configurations, (originally shown in Figure 3.5) shown here for convenience in interpreting Figure 4.1. . . . . . . . . . . 26

4.3 Relative magnitude and phase between antenna 1 and antenna 5. Note that the phase appears more coherent at lower frequencies. . . . . . . . 27

4.4 Relative magnitude and phase between antenna 1 and antenna 6 . Note that the phase does not appear as coherent at lower frequencies as it did in Figure $4.3 . \ldots \ldots$. . . . . . . . . . . . . . . 
4.5 Antennas from configuration 2 but with different ground spacings. This shows a strong relative spatial coherence between closer antennas. 29

4.6 Time domain of $1 \mathrm{kHz}$ wide channel centered on the transmission frequency. . . . . . . . . . . . . . . . . . . 31

4.7 Rolling average of window length 200 samples, for a $3.8 \mathrm{MHz}$ transmission. . . . . . . . . . . . . . . . . . 32

4.8 Mesh (surface) of $3.8 \mathrm{MHz}$ signal received across phased array. . . . . . 32

4.9 Animation frames of mesh from a $3.8 \mathrm{MHz}$ signal showing a peak, consistent across the data from each antenna pair, travelling acaross the array. . . . . . . . . . . . . . . . . 34

4.9 Animation frames of mesh from a 3.8 MHz signal showing a peak, consistent across the data from each antenna pair, travelling acaross the array (cont.). . . . . . . . . . . . . . . . . . . . . 35

4.9 Animation frames of mesh from a 3.8 MHz signal showing a peak, consistent across the data from each antenna pair, travelling acaross the array (cont.). . . . . . . . . . . . . . . . . . . . . 36

4.9 Animation frames of mesh from a 3.8 $\mathrm{MHz}$ signal showing a peak, consistent across the data from each antenna pair, travelling acaross the array (cont.). . . . . . . . . . . . . . . . . . .

4.10 FFTs of all relative phase data showing no apparent frequencies of ionospheric waves under $1.25 \mathrm{~Hz}$.

4.11 Ionospheric reflection height as function of physical geometry and RX-antenna phase difference. . . . . . . . . . . . . .

4.12 Ionospheric height versus phase difference for a $3.7 \mathrm{MHz}$ transmission received over a $60 \mathrm{~m}$ ground spacing, assuming a planar wave front. . . . . . . . . . . . . . . . .

4.13 Ionospheric reflection height for a $3.7 \mathrm{MHz} \mathrm{CW}$ transmission assuming a planar wavefront. . . . . . . . . . . . . .

A.1 Mast support. . . . . . . . . . . . . . . . . . . 46

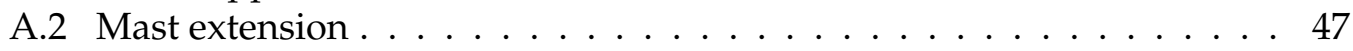

A.3 First system setup on roof of ELW. . . . . . . . . . . . . . 48

A.4 Antenna 4 on the UVic KiwiSDR. A strong signal $(-85 \mathrm{dBm})$ is apparent around $3.84 \mathrm{MHz} . \ldots \ldots \ldots \ldots$. . . . . . . . . . . .

A.5 Map of the experimental area. Red circles indicate antenna locations proposed by Dr. Stephen Harrison, the red marker indicates the shed which would provide access to power. . . . . . . . . . . . 50

A.6 Plan view of eight planned antenna orientations. . . . . . . . . . . . 51

B.1 Multipath channel model results. . . . . . . . . . . . . . . . . . 54

B.2 Watterson model simulation. . . . . . . . . . . . . . 55

B.3 Experiment length correlation and single peak detail. . . . . . . . . 56

B.4 Overlaid correlation peaks. . . . . . . . . . . . . . 57

B.5 Phase (orange, right y-axis) and magnitude (blue, left y-axis) of the correlation zoomed in on a single peak. . . . . . . . . . . . . 57

B.6 Phase of the correlation over an entire transmission. . . . . . . . . . 58

D.1 The architecture of the GNU Radio interface with the Flex radio. . . . . 62

D.2 The gr-flex block being used to visualize a waterfall and FFT in GNU Radio. . . . . . . . . . . . . . . . . . . . . 63 


\section{List of Tables}

3.1 ENI 2100L RF Power Amplifier specifications [25] . . . . . . . . . . . 17

3.2 Beamformer parameters used during the DRAO-UViip experiment. . . 19

3.3 Antenna configurations. . . . . . . . . . . . . . 23

A.1 Army surplus tent-pole dimensions . . . . . . . . . . . . . . . . . . 45

A.2 Values from the antenna tests. . . . . . . . . . . . . . . . 47

A.3 Wavelengths relevant to the experiment $\left(c \approx 3 \times 10^{8}\right) \ldots \ldots 50$

B.1 Watterson model simulation inputs. . . . . . . . . . . . . 55 


\section{Acknowledgements}

Without the help of these people, the work done for this thesis would have never happened.

Dr. Rodney Herring for supporting and guiding me through this research. From my time as an undergraduate, he has put aside time to mentor me and let me explore the world of research.

Dr. Peter Driessen for adopting me into his laboratory and guiding me through both school and life.

Dr. Stephen Harrison for patiently answering my questions and supporting the project from afar.

DRAO personnel for hosting me during the data collection.

Emily Jones for letting me wander but always guiding me back.

My parents for their belief in me and the family time spent together which always brought me back to the research with fresh ideas and enthusiasm.

Dr. Ahmed Youssef for asking me why my thesis wasn't done yet, and showing me the value of a clean whiteboard.

Colter McQuay \& Peter Kremler for making my time in the lab far more fun than the science alone. 


\title{
List of Abbreviations
}

\author{
ACM Array Covariance Matrix \\ ADC Analog to Digital Converter \\ BW Bandwidth \\ DRAO Dominon Radio Astrophysical Observatory \\ HF High Frequency \\ LFM Linear Frequency Modulation \\ LWA Long Wavelength Array \\ RF Radio Frequency \\ RFI Radio Frequency Interference \\ SDR Software Defined Radio \\ SNR Signal to Noise Ratio \\ UViip University of Victoria ionospheric imaging project
}




\section{Chapter 1}

\section{Introduction}

\subsection{Introduction}

The ionosphere is a region of the atmosphere consisting of layers of particles that are ionized by incoming solar radiation. This entity, which is typically considered to be several distinct layers based on ion densities, is also an extremely sensitive instrument for measuring both terrestrial and solar behavior. This is because significant events such as volcanoes, earthquakes and solar flares create measurable waves on the surface of the ionosphere [1]. Weaker signatures can also be found relating to smaller events such as extreme weather, and large scale explosions.

Software defined radio (SDR) is an increasingly popular paradigm for building radio frequency transmitters and receivers. Analog systems used to be relatively single purpose, working on specified (by design) frequency bands and delivering analog wave forms to the user. As hardware has become smaller and better able to handle a variety of frequencies and bandwidths, the field has shifted towards a software defined approach. For example, in a receiver the hardware is able to be used across a variety of frequency bands (sometimes simultaneously) by sending everything the system receives as a radio wave to an analog-to-digital converter (ADC) after which a computer processes the now discrete data. This capability allows relatively non-specialized hardware to work in very specialized applications.

This thesis investigates the potential to study ionospheric disturbances by using software defined radio technology. Studies discussed in Chapter 2 show that there are precursors to major natural events like earthquakes which are apparent in the ionosphere up to five days before the event occurs [2]. Considering that the currently existing earthquake warning systems are only able to provide $90 \mathrm{~s}$ of warning before an earthquake hits [3], a technology able to offer even a few days of warning would be invaluable.

\subsection{Motivation}

The University of Victoria ionospheric imaging project (UViip) is a collaborative research group between the mechanical and electrical engineering departments. The goal of UViip is to characterize disturbances existing in the ionosphere using SDR technology. Eventually, the intent is for this to allow for a new weather analysis and natural disaster prediction technique.

In order to do this, an experiment has been devised. The premise is that a radio wave is transmitted from some site at perfectly vertical incidence, the radio wave travels to the ionosphere which reflects the radio wave back to earth where it is received on a second site. The second site consists of an array of radio antennas. If no ionospheric disturbances exist, the radio wave should take the same amount of 
time to arrive at each receiver (each path length is the same). However, if ionospheric disturbances do exist, they should change some path lengths and each receiver in the array would have a different time delay.

To analogize this system with traditional imaging, the receiving array size (number of receiving antennas) dictates the images pixel-count. Each received radio wave acts as the value of one of those pixels. If the array is large enough to obtain a relatively high resolution image, it may be possible to view waves passing above the system, as well as triangulate them. This capacity would increase if multiple arrays were near each other, since the different images could also help with triangulating the source of disturbances.

Based on literature reviewed in Chapter 2 it is apparent that large ionospheric disturbances are able to travel through the ionosphere for at least $6000 \mathrm{~km}$ [4]. Assuming that each receiver array is the center of a $2000 \mathrm{~km}$ imaging radius (safety factor of 3 , in order to consider smaller disturbances), all of Canada could potentially be imaged with 10 of these systems.

Before developing such a large array, a feasibility study was designed to collect some preliminary data and practice building the necessary system components (a shorter feasibility study was conducted beforehand and is described in Appendix B). The feasibility study is built on the same concept as that described above but with a much smaller and mobile receiver array.

This thesis discusses the feasibility study done to test whether current SDR arrays are able to meet the needs of UViip, and whether any ionospheric disturbances could be imaged with a smaller, mobile measuring system. Some relevant design parameters of the feasibility study are as follows.

- Operation in the HF (high frequency) radio bands (specifically the 3.5-4 MHz amateur radio band). The $3.5-4 \mathrm{MHz}$ is doubly useful since it is available to people with easily attainable amateur radio licenses, and the frequency range which reflects off of the ionosphere [5].

- Synchronous processing of multiple antenna inputs. This will allow comparison between antennas (an important considering for imaging the ionosphere). Without this synchronicity, there is no guarantee that the pixels in the generated image were captured at the same time.

\subsection{Contributions}

This thesis focuses on the development of a phased-array built to image the ionosphere.

\subsubsection{Development and running of an experiment at DRAO}

An experiment was developed to make use of space and instrumentation at DRAO (Dominion Radio Astrophysical Observatory). The experimental design included the instrumentation as well as the layout and methods to be used when operating the phased-array. Once designed, the entire array and supporting physical infrastructure were built at UVic and tested. This included antennas, matched-length cabling, mobile light-weight tripods to mount the antennas on, a power management system, and appropriate filters and amplifiers. The phased-array was transported to DRAO and setup. It was tested and adjusted before multiple data-collection sessions were run over three days. 


\subsubsection{Preliminary data analysis}

The data collected was analyzed in a variety of ways with the purpose of checking whether the phased-array had been able to image the ionosphere. In the process of proving that the system worked as predicted, a large repository of visualization software was written. As well, a novel method for measuring ionospheric height using two phased antennas was developed.

\subsubsection{Miscellaneous}

The appendices of this thesis include a substantial amount of work done in preparing for the DRAO experiment as well as a preliminary study and set of simulations done to check the viability of the overall project objective. As well, there are contributions to various research efforts outlined in the appendices of this thesis, not all of which are directly related to the thesis' focus. They are included for the sake of completeness in documenting work done.

\subsubsection{Summary of contributions}

In summary, the main contributions of this thesis are:

- design of an experiment to image the ionosphere in a novel way,

- building a phased-array and the supporting infrastructure,

- running the designed experiment at DRAO,

- analyzing the data obtained, and in the process creating a new method for measuring ionospheric height.

\subsection{Structure of the thesis}

The chapters and appendices of this thesis are briefly described below:

Chapter 2 A review of relevant literature.

Chapter 3 The methods used and preparation carried out to run the experiment.

Chapter 4 The results of the experiment and discussion.

Chapter 5 Concluding remarks and recommendations for future work.

Appendix A Description of work done in building the equipment used for this thesis.

Appendix B Description of additional work done in support of UViip while not contributing directly to this thesis' experiment.

Appendix C A proposal and its results for future work to be done on the Long Wavelength Array (LWA) in New Mexico.

Appendix D Description of miscellaneous work done not directly contributing to the UViip research effort. 


\section{Chapter 2}

\section{Literature Review}

This chapter includes a brief summary of atmospheric and ionospheric structure, describes a series of studies on ionospheric disturbing events, and looks into research being done with a similar purpose to UViip.

\subsection{Contextual vocabulary}

There have been many studies on the relation between disturbing events and ionospheric responses [6]. The convention has been established that a 'region' is a band of atmosphere (for example, the $D$ region, $E$ region and $F$ region) of roughly specified height from the ground while a 'layer' is a sub-band of ionized plasma within a region (for examples, the F1-layer and the F2-layer) [1], [6]. This is depicted in Figure 2.1 and moving forward in this writing, the same convention is adopted. As well, when discussing any waves travelling throughout the ionosphere, they are referred to 'ionospheric disturbances', or simply 'disturbances'. Following, the triggering events related to the disturbances are referred to as 'disturbing events' or just 'events'.

\subsection{Ionospheric physics and chemistry}

The ionosphere exists because of solar radiation. As the sun irradiates the earth, the gases in the upper ionospheric region are ionized into free electrons and ions by $\mathrm{x}$-rays and ultra-violet rays. These high energy wavelengths do not penetrate deep

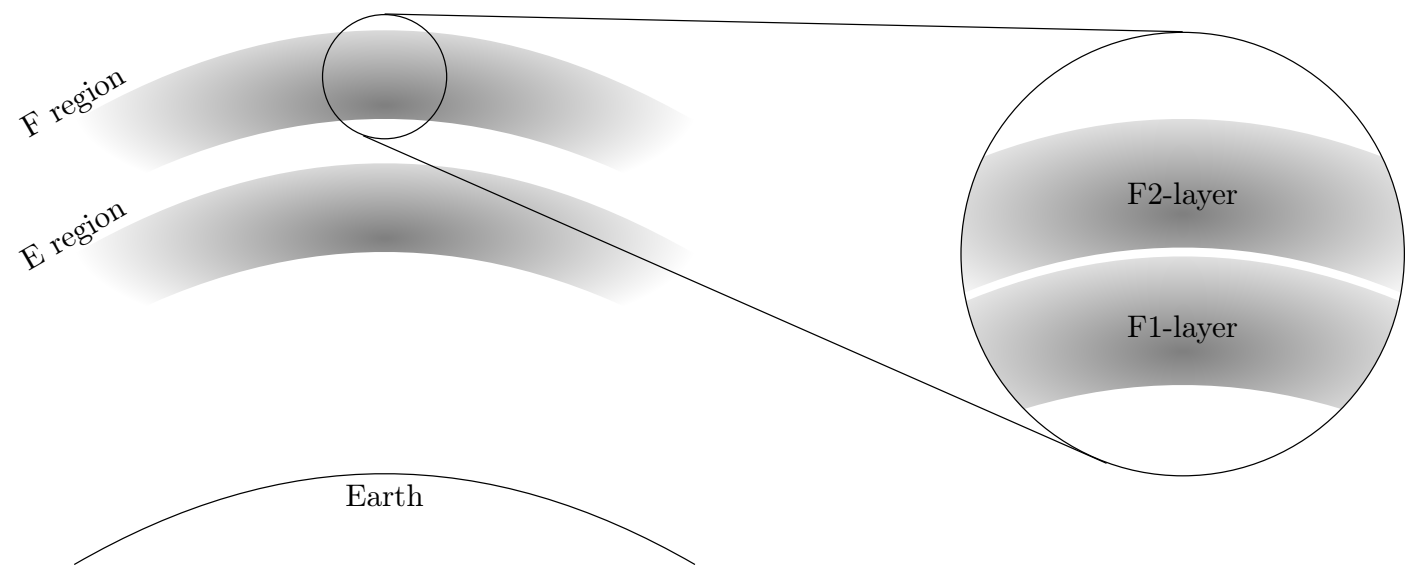

FIGURE 2.1: Ionospheric $\mathrm{E}$ and $\mathrm{F}$ regions showing that the F-region consists of F1 and F2 layers. 

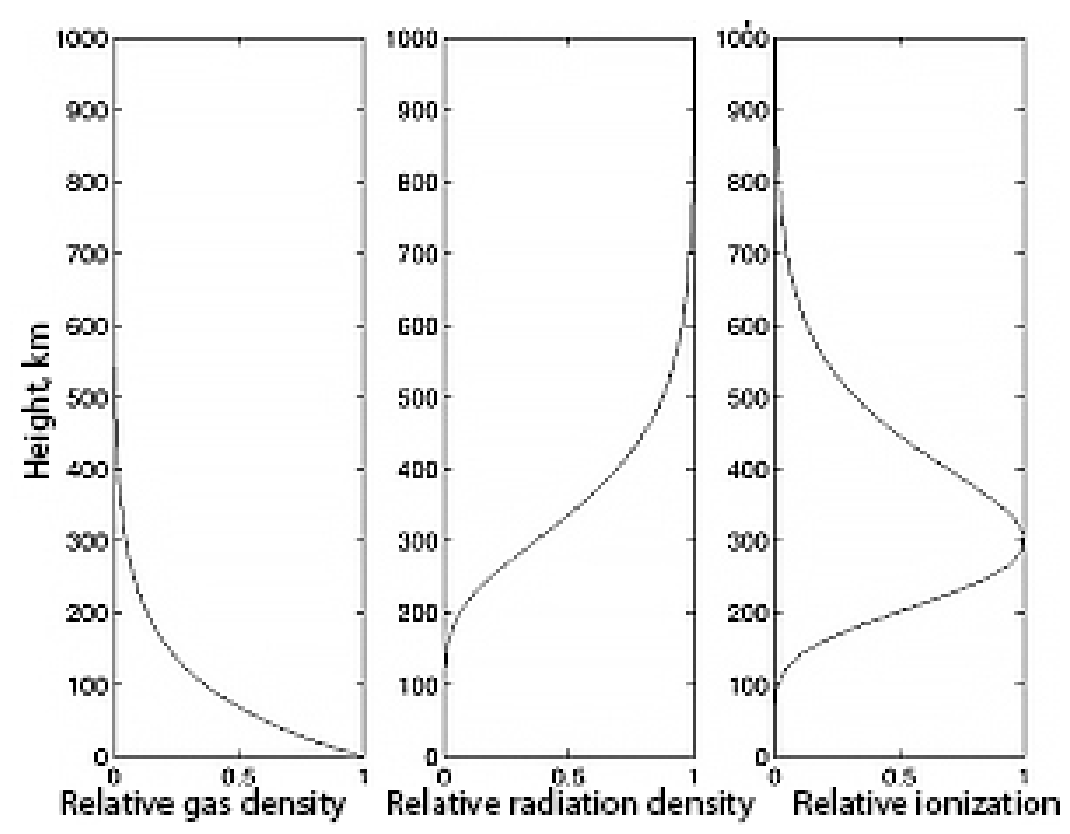

FIGURE 2.2: Relative gas density, radiation intensity, and ionization of the atmosphere vs height [7].

into the atmosphere which is why the surface of the earth is habitable. Figure 2.2 shows the relationships between gas density, radiation intensity and ionization.

It follows the understanding of solar radiation being the cause of atmospheric ionization that the free electron density is highest during periods of increased radiation exposure, for example during the summer months and during daytime hours. The peak density in the F2-layer is often written more compactly as NmF2. It can be read as the electron density $(N)$ maximum $(m)$ in the F2-layer $(F 2)$. The corresponding height of this point is written as $h m F 2$.

This peak exhibits known behaviors [6]. They are summarized as follows.

1. Up to $h m F 2$, the atomic ionization $(q)$ and recombination $(\beta)$ rates balance each other, but with enough difference that their ratio, $\frac{q}{\beta}$ increases. This means that $N$ increases as well.

2. Above $h m F 2, N$ stops growing, and starts to exponentially decay with height as gravity decreases and the ion density decreases.

3. $h m F 2$ exists along an atmospheric isobar.

Note that the heights in discussion are all actual heights, and not the virtual height as read by common ionograms (illustrated in Figure 2.3). The ionosphere acts as a refractive lens, not a reflective lens. This is also shown in Figure 2.3. As well, each of Figures 2.1 and 2.3 shows defined boundaries for the ionospheric zones, but these 'boundaries' are more diffuse than illustrated. Note that the skip distance (distance the radio wave travels without contacting the earth) is also marked on Figure 2.3.

As mentioned, the ionosphere acts as a refractive lens on electromagnetic waves. As the waves approach an ionized area with a high free electron density, the electrons are excited and re-direct the signal at an angle proportional to that density. If the wave is entering an area of increasing ionization, the refraction angle will 


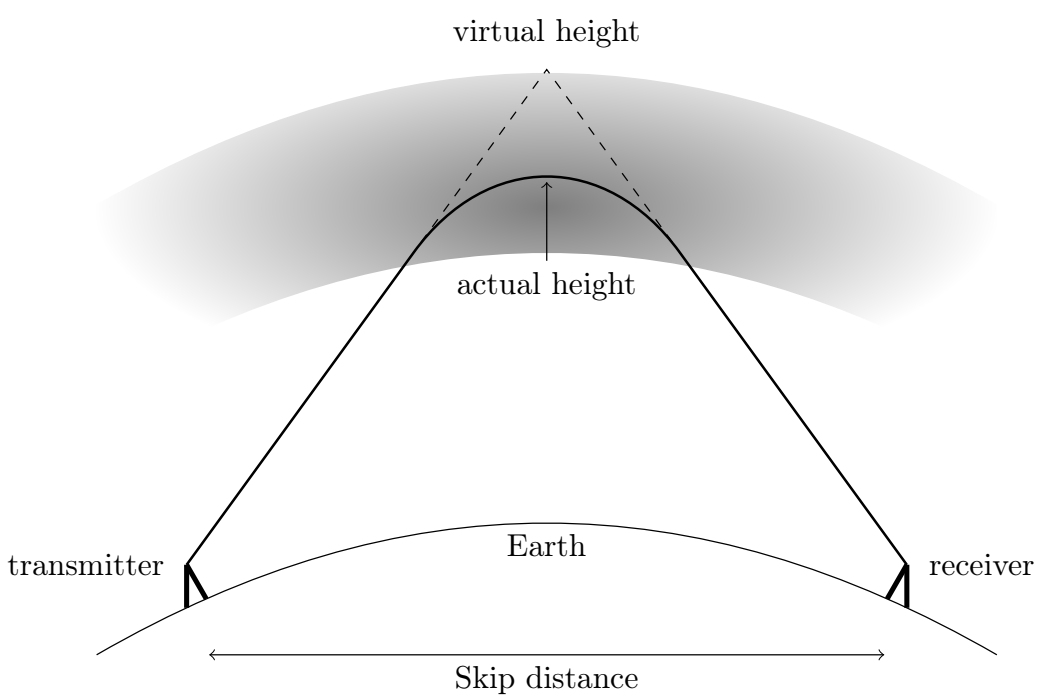

FIGURE 2.3: Difference between actual refraction height and virtual reflection height.

sharpen. The amount of refraction is a function of ion density, as well as the frequency and angle of incidence of the impinging wave. If enough refraction occurs, the wave will bend right around and return to Earth as if it was reflected. In general this occurs for waves of frequency below $30 \mathrm{MHz}$, but at near vertical incidence is further restricted to $10 \mathrm{MHz}$ or less depending on the ionospheric characteristics at that time of day/year, as explained in Section 2.3.

Ionospheric heights are often measured by ionosondes. An ionosonde transmits frequency sweeps vertically and receives them at the same location. Short sweeps are transmitted, reflected by the ionosphere at whatever its critical frequency (the frequency above which a radio wave passes through the ionosphere instead of reflecting off of it) may be, and when the time delay between the transmitted and received sweeps is converted to a travelled path length, an ionogram is created. Figure 2.4 shows an example ionogram. Note that there are two F2 readings: one is the original transmission while the second is an orthogonal polarized copy of it.

\subsection{Ionospheric variance}

As mentioned in Section 2.2, there are variations in the heights and electron densities of the various ionospheric layers caused by the periodic nature of solar irradiation in any local area. During the night, the F1-layer merges into the F2-layer leaving only the F2-layer until the sun begins its daytime ionization. The F2-layer continues throughout the night because it is the furthest from the surface of the earth and so has the lowest ion density, and thus the lowest rate of atomic recombination. Above $h m F 2, N$ decreases exponentially meaning that the recombination of ions and electrons is much slower and so exists throughout the night [1]. It does become weaker throughout the night but, as evidenced by 24 -hour radio communication and commercial radio stations, radio waves are able to be refracted back to earth despite this waning of electron density.

Expanding the time scale, an annual cycle can be observed in which the longer daily radiation time during summer causes higher mean ionization values in all the ionospheric layers. This trend then reverses itself in winter time. 


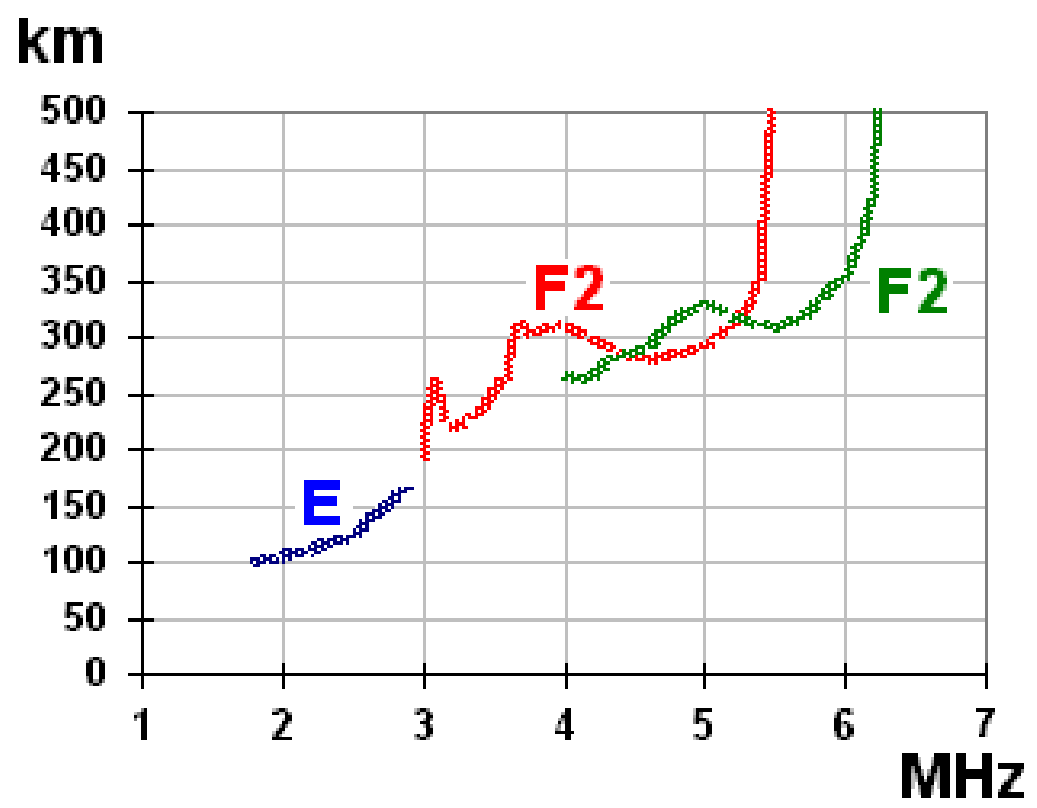

FIGURE 2.4: Ionogram showing ionospheric height [8]. The two F2 curves are due to polarization of the radiowave by the ionosphere.

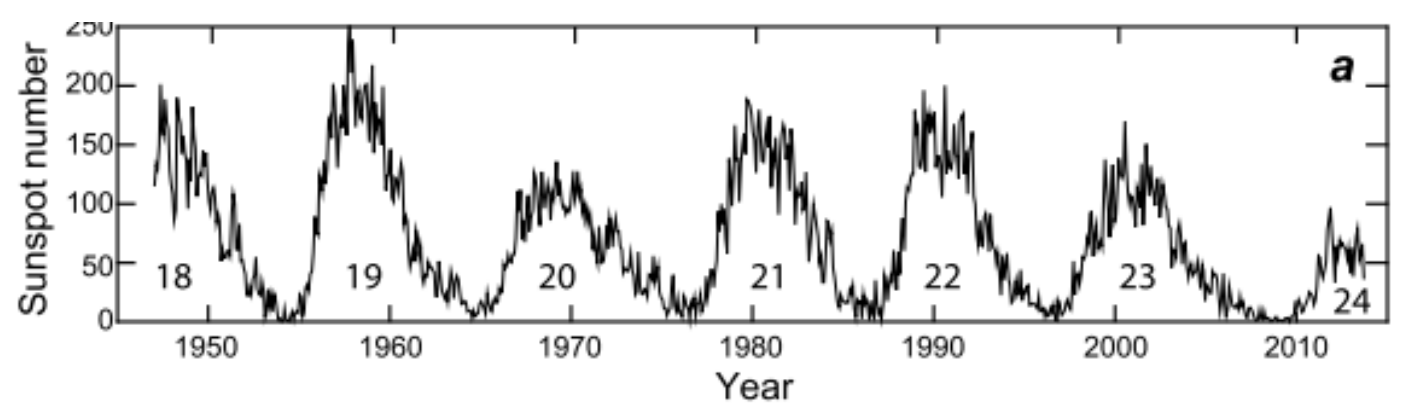

FIGURE 2.5: Historical solar activity [9] showing an 11-year solar cycle.

Another almost-periodic variance is the 11-year solar cycle. Not only is this a cause of steady change in mean irradiation values but it changes the impact of sunspot/solar flare activity in the ionosphere, a major disturber of the F2-layer. Figure 2.5 shows the sunspot number over time, which is frequently used to indicate solar activity [9]. The 11-year cycle is clear, and the numbers in each cycle indicate the cycle number. As of this writing the cycle 24 is the current one and the minima was in 2010.

Characterizing these relatively steady periodic changes is critical to the characterization of disturbing events as they indicate a baseline function describing what is normal and what is abnormal in the ionosphere.

\subsection{Ionospheric disturbances}

In concluding his 1998 paper, Rishbeth recommends some guiding principles for ionospheric research. He reminds the reader that in the F2-layer of interest, ionospheric parameters (remembering the isobar, and extrapolating from that example) 
vary faster vertically than horizontally. However, the vertical motions are generally slower than the horizontal speeds. This is easy to visualize when imagining a large hurricane with a rapid wind speed pulling the ionosphere along with a horizontal shearing force while causing less change vertically. This is generally due to the larger energetic inertia on the layer due to gravity. It's important to bear this in mind when discussing ionosphere disturbing events since the radio wave refraction happens with a greater component of the travel being vertical than in plane with the earth's surface. That ratio obviously changes with the skip distance of the radio wave, but in general the trend will hold.

Rishbeth provides a high level look at the topic of ionospheric disturbing events in his 2006 paper. The primary findings and discussion in this paper have to do exclusively with the F2-layer in the ionosphere. It was concluded that although large natural and man-made events, including severe weather systems and bombs, definitely result in oscillations propagating through the ionosphere, there was a distinct lack of evidence attributing any ionospheric changes to the gentler day-to-day weather patterns.

On the topic of large, natural and man-made events, it was also noted that these ionospheric effects were quick to dissipate over both time and distance. To resolve a disturbing event, sensor proximity to the event will be extremely relevant.

\subsubsection{Earthquakes}

Earthquakes are well reported to cause disturbances in the $F$ region of the atmosphere, and many times are reported to show precursors up to 5-days prior to the earthquake itself [2]. This makes the study of any potential predictive characteristics in these precursors incredibly valuable. Bleeding edge technology exists in the Juan de Fuca Strait, Canada to offer 30-90 seconds of warning by means of a sea-bed seismic sensor network [10]. Obviously a system that provides a multi-day warning and works with minimal instrumentation by means of ionospheric characterizations, would be a significant improvement.

The mechanism that transmits the earthquake precursors, post-cursors, or event information to the ionosphere is not well-known. It is hypothesized that the seismic stresses that mount before the tectonic shifting create a strong electric field which acts on the charged particles in the ionosphere [11]. This would mean that the characteristics of the electric field would depend on the rock type through which it propagates [12]. It has also been thought that ions themselves could be transported from lower atmosphere to the ionosphere by these same electric fields [13].

This theory could be verified by sampling the gas ratios in the $F$ region during an event to see whether any of the gasses are higher or lower in the atmosphere than usual. Although interesting, it would be a difficult experiment to execute, and does not impact the characterization efforts by UViip.

An entire book has been published discussing prediction methods for earthquakes by studying related electromagnetic phenomena [14]. Commentary is made that "short-term earthquake prediction has been found to be impossible using conventional methods with seismometers but that it is becoming possible using electromagnetic phenomena by radio techniques". The book works through a variety of measurement techniques ranging from satellite observations, through atmospheric observations via a range of radio frequencies, to geophysical DC signals caused by the earthquake itself.

A Russian study found that the earthquakes electric field was most noticeable during the nighttime and in the E Region of the atmosphere [11]. This implies that 


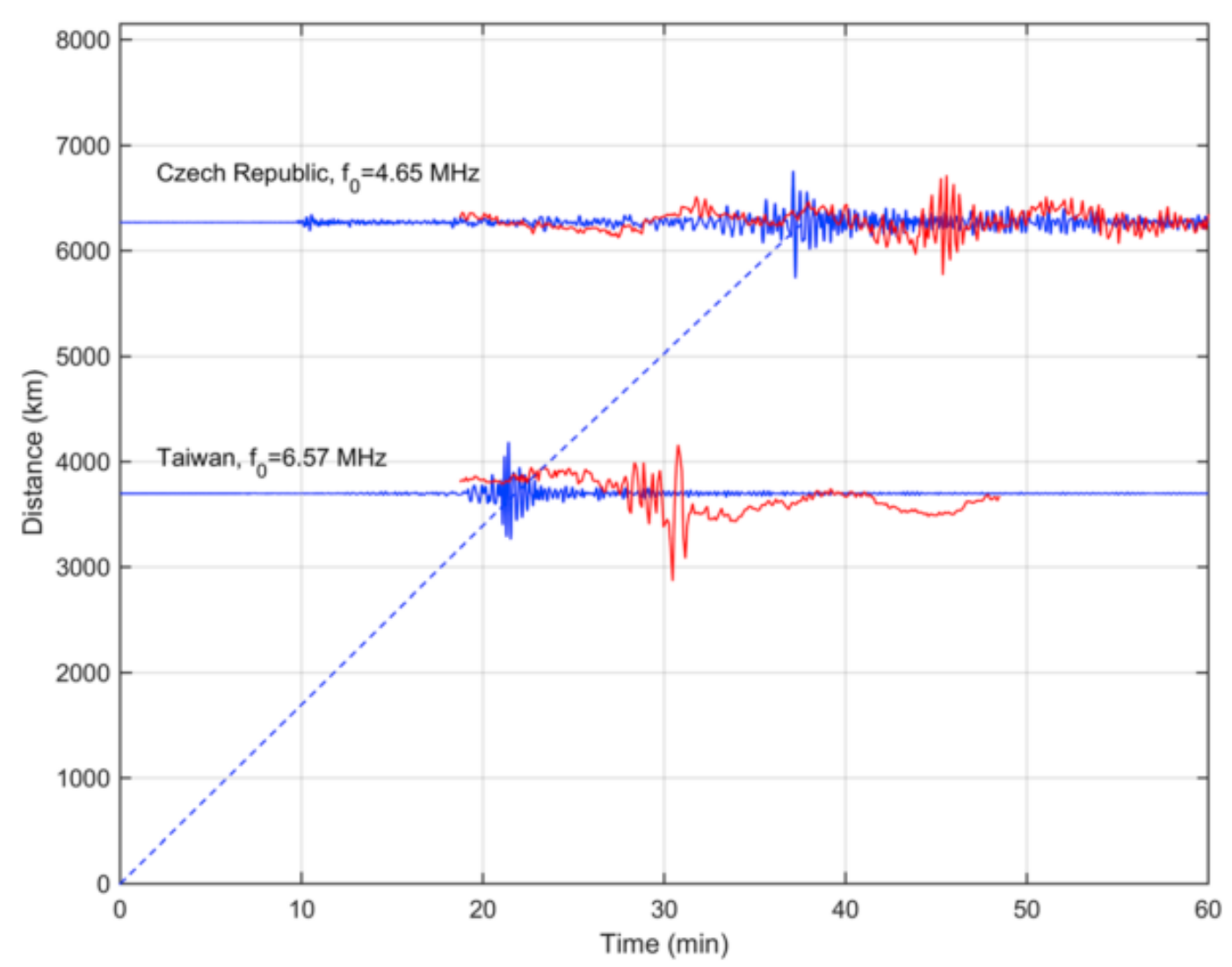

FIGURE 2.6: Time delay results between the ground response (blue) and ionosphere response (red) to the 2015 earthquake in Nepal from two different locations, giving a measure of $10000 \mathrm{~km} / \mathrm{h}$ along the ground and $8000 \mathrm{~km} / \mathrm{hr}$ through the ionosphere [4].

an earthquake warning system would have to be implemented to survey multiple regions in the ionosphere and characterize them all, since other studies have focused on the $F$ region. That is, unless one region can be successfully characterized with a high probability of success.

An entirely different mechanism for an ionospheric signature was reported after the Nepal earthquake which struck in 2015 [4]. The study found that ionospheric signatures, likely produced by the magnitude M7.8 earthquake, were induced by long period infra sound waves. The waves were excited by ground motion during the quake and propagated largely uninterrupted to the $F$ region where they were immediately damped and the energy from them passed into ionospheric waves. These signatures were all recorded during the actual event from two locations, Taiwan and the Czech Republic (it supports the previous statement on the ionospheric disturbances damping in plane with the isobar that "two more distant measurements did not reveal a measurable response" [4]). Figure 2.6 shows the time delay between ground-based seismographic readings and the ionospheric signature.

Evidently, there have been a lot of qualitative studies on earthquake signatures of one form or another. In order to characterize the precursors, quantifiable data will be needed. To this end, work has been done on proposed statistical tests for these precursors [2]. Seismographic data was collected for all earthquakes in the Taiwan area of magnitude greater than 5.0 between 1994 and 1999. From this set, they focused on earthquakes of magnitude greater than 5.0 whose centers were within 500 

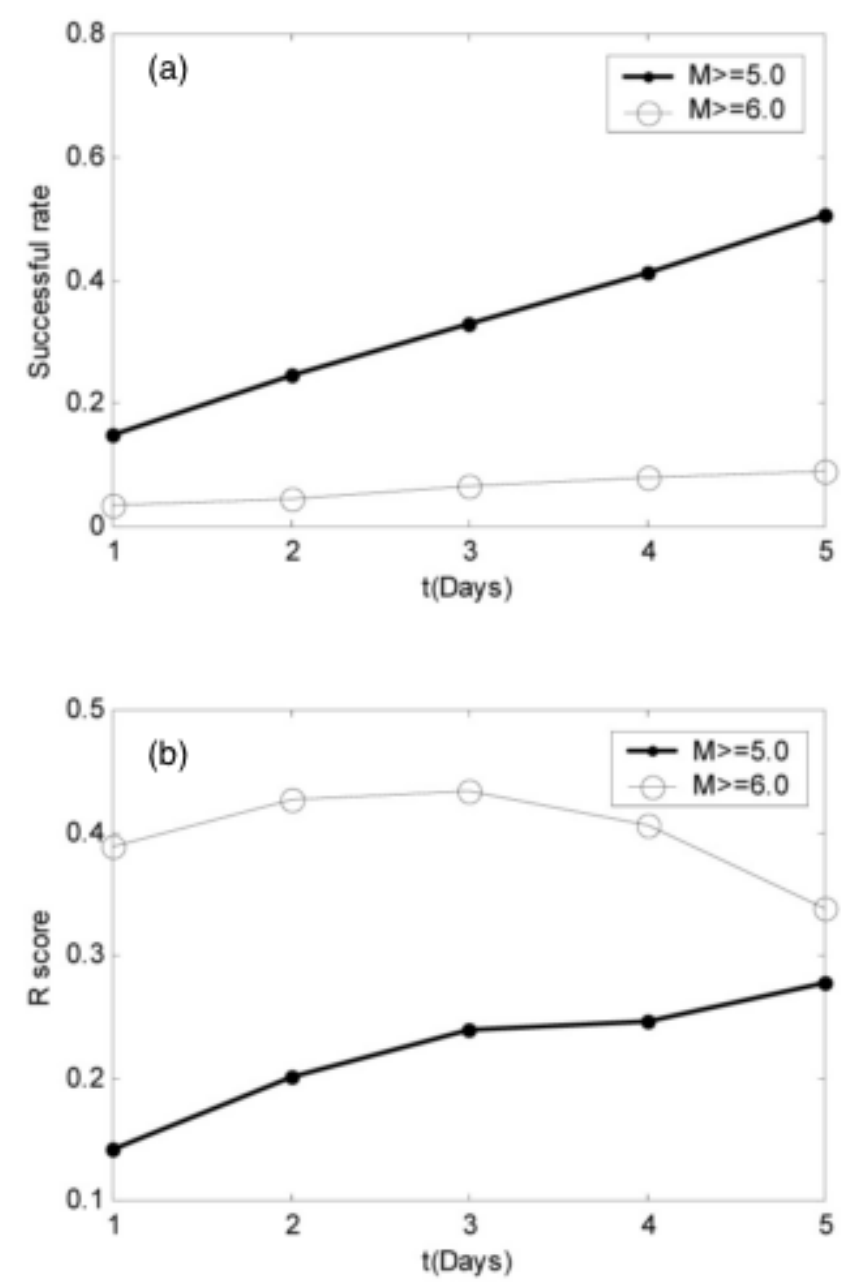

FIGURE 2.7: (a) success rate and (b) probability score of precursors for earthquakes of $\mathrm{M}>5.0$ and $\mathrm{M}>6.0$ respectively [adapted from Chen, Liu, Tsai, et al., 2004 [2]].

$\mathrm{km}$ of the radio signalling station. It was found that there were 307 ionospheric disturbances and 170 earthquakes during this time period. A probability curve was set up in which the number of anomalies that preceded earthquakes, the number of anomalies that preceded no earthquakes and the number of earthquakes with no precursor were compared to find a proportion of 'false alarms'. It was found that $73 \%$ of earthquakes with $M>5.0$ had foF 2 anomalies within the 5 days prior to the event. Figure 2.7 shows the success rate (Figure 2.7a) as well as the probabilistic (R) score (Figure 2.7b). It is also interesting that although the success rate rose leading up to the earthquake, so did the failure rate and so the probabilistic rate was maximized at 3 days prior.

In order to fairly analyze the potential for high-probability earthquake precursor characterization, it is important to review studies which have been critical of some characterization methods. It has been argued that basing precursor characterization on a 40-minute prior TEC (total electron count in a constant volume of cross-section $=1 \mathrm{~m}^{2}$ ) increase as claimed by Heki ([15]) was erroneously done [16]. An important clarification is that the critics do not deny the existence of potentially characterizing the precursors, but believe that the 40-minute prior warning signal 
is an artifact of the data-fitting method used. It will be useful for future characterization efforts to deeply consider both studies in order to avoid any possible dissent over method validity.

\subsubsection{Weather and storms}

Most of the research into earthquake signatures has gone into radio wave propagation via the F2-layer, however there have been studies of other ionospheric regions returning useful meteorological correlations. A decade long study, done at the $\mathrm{Zi}$ Ka Wei Observatory in Shanghai, China, was completed in the 1940s and was able to successfully predict weather patterns by transmitting critical frequencies for each of the atmospheres $D$ region, $F$ region, and F2-layer [17]. If the critical frequency echoed (i.e. returned to a ground antenna at the observatory), it was found to be related to the movement of the global air masses. The study found that when there was an echo from the $E$ region, the maritime air mass was either overhead or would be soon. If the $F$ region returned an echo, the polar air mass would be the weather pattern, and if the F2-layer returned an echo, the tropical air mass would do the same. The movement of these air masses are directly related to the movement of typhoons in the southern hemisphere and it was accurately used as a predictor of impending typhoon danger in both Shanghai and Hong Kong.

\subsubsection{Man-made disturbances}

In a different area of study it was found that large explosions, including underground nuclear experiments, emit large enough infra sound waves that they push at the ionosphere from below [18]. This implies a military or security technology application of ionospheric characterizations. The advantage of studying infra sound waves is that due to the direction of the atmosphere's temperature gradient (warmer close to the earth), infra sound waves are naturally focused vertically and will quickly dampen in the $F$ region [18]. Ground-based infra sound wave sensors have an inherently faster response time than ionospheric readings and so to be competitive in this field the ionospheric readings would have to provide a higher quality result of some sort.

\subsection{Research efforts to image ionospheric disturbances}

The ionospheric disturbances have been an area of study for decades but with no concrete results. There are currently several high profile research groups working on the issue. One such effort is being made out of New Mexico at the LWA (Long Wavelength Array) observatory. LWA1 is the first of multiple planned antenna arrays designed for cosmic observation. The observatory is 256 phase-coherent dipole antennas operating primarily above $10 \mathrm{MHz}$ [19]. PASI (Passive All-Sky Imager), is a new device (or rather, method of managing the existing array). The purpose of the system is to demonstrate a real-time radar-based ionospheric imager. Their ionospheric readings were primarily restricted to the $E$ region because of the higher frequencies in use. As such, their readings were sporadic. The focus of the research at that point was to check the validity of the system and so no effort was put into characterizing the dense clouds (sporadic- $E$ region) that were imaged. They were able to map the spatial distributions and vertical drift speeds of these clouds, but put more effort into measuring the coherence of the array. Looking forward, this group will have access to the 52 planned observatories of a similar size to be spread 
across New Mexico. This will give the group a very powerful tool with which to image the ionosphere.

An extension of the same group working out of the same observatory (LWA1) published results on the hardware in use and how the massive data volume is being managed real-time [20]. They also discuss RFI and comment on the exceptionally clean operating environment they find in the protected radio bands specified for radio astronomy. A newly developed 'lightening mode' is also described in which they increase their sampling rate to $200 \mathrm{~Hz}$ (5ms per frame) which allows them to image the electric charge breakdown before a lightening strike as well as the actual flash of the strike. Both of the LWA1 papers conclude by suggesting that the stations could easily be used for more advanced ionospheric imaging.

Imaging lightening can be considered a good starting point for other ionospheric disturbance characterization because the source is known. If a secondary array nearby is imaging near-vertical, it may be possible to image the ionospheric disturbance caused by the lightening.

A Chinese/Japanese collaboration is apparently currently working on 3D ionospheric imaging. They captured 3D images of apparent ionospheric anomalies during the 30 minutes before the 2015 earthquake (magnitude 8.3) which hit Illapel, Chile [21]. Their method is to calculate the electron density at various heights across a square patch of sky. They used a total of 211 GPS stations spread across Chile and Argentina to collect the data (Figure 2.8). The output data shows a clear shift from normal electron density across a variety of ionospheric heights with the difference increasing as the time before the earthquake shortens (Figure 2.9). Importantly, this study does not make any effort to characterize the imaged disturbance with the goal of using it to predict future earthquakes.

\subsection{Concluding remarks}

In this literature review a relatively deep discussion on the ionospheric makeup is done including the various causes of ionization and the importance of understanding the electron dynamics within it. This leads to a greater understanding of the maximum electron density in, for example the F2-layer $(f m F 2)$ and the corresponding height of this density $h m F 2$. These values are important to understand in order to understand radio wave propagation through and in these areas and the potential mechanisms by which waves can travel through the ionosphere. This naturally leads into a discussion of periodic variances to the ionospheric electron densities $N$. These variances are primarily recognized to be related to the daily, annual, and 11-year solar cycles. Understanding these topics allows for a discussion on previous studies to do with ionospheric disturbances. The disturbances discussed are earthquakes, which have many times been proven to have stronger precursors as the magnitude of the earthquakes increase. It follows that having an ionosonde with both high frequency and sampling rate resolutions would provide precursors with a higher probability that a lower resolution ionosonde. Weather and storms are discussed as well. In particular one study which concluded in 1950 immediately shows promise for predicting day-by-day weather patterns. Man-made disturbances are briefly covered and it is noted that although infrasound shifts due to underground nuclear explosions can be seen in the ionosphere, there already exists a ground based network of sensors to provide this warning service. Finally, several efforts being made by other research groups to image ionospheric disturbances are discussed. A summary of this entire literature review can be taken from Rishbeth's reminder, "One should 


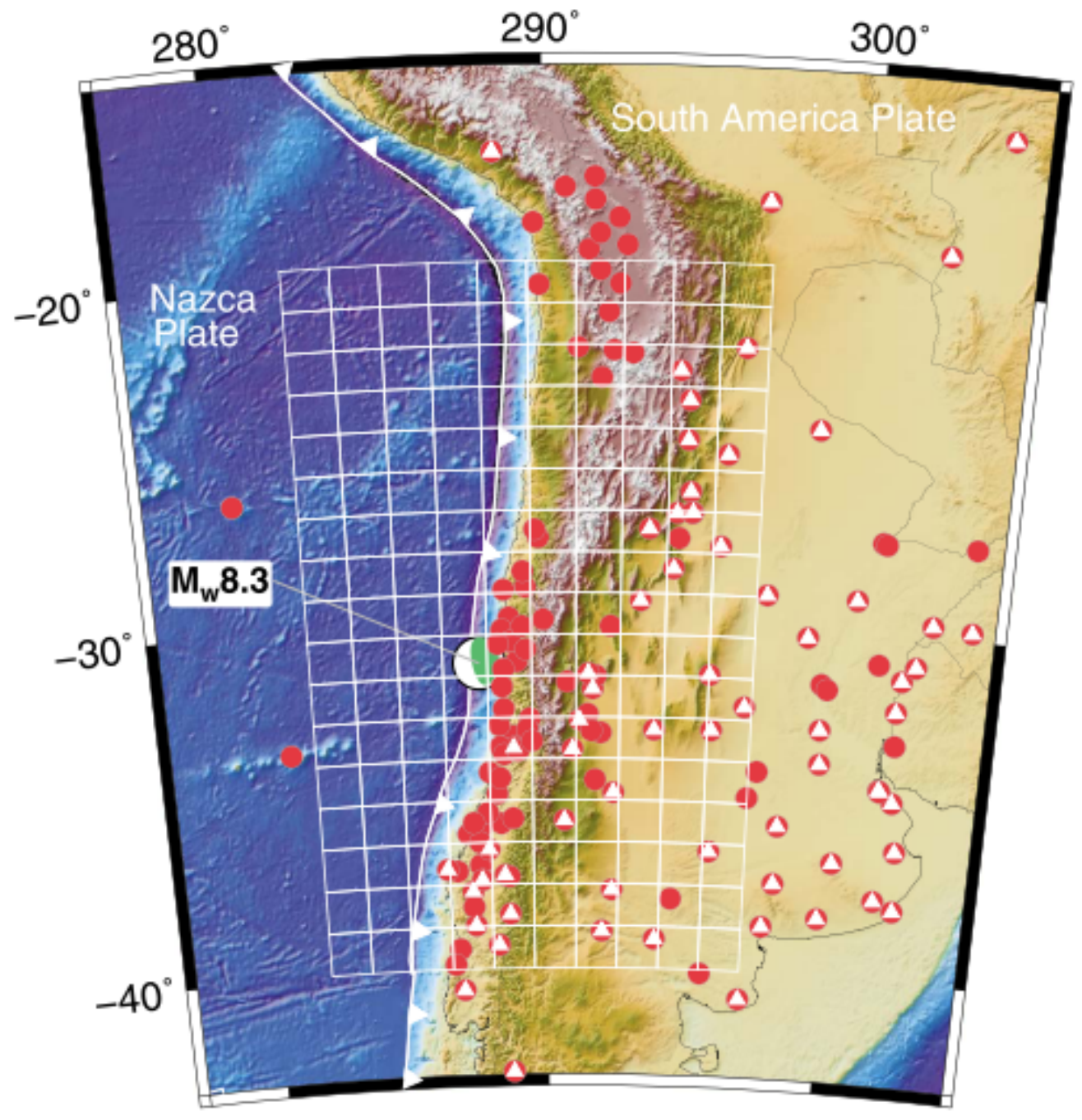

FIGURE 2.8: GPS stations used to image earthquake precursors prior to Chile's major 2015 earthquake. Red dots show GPS stations, white triangles in red dots show GPS/GLONASS stations, the green dot is the earthquake epicenter [21]. 


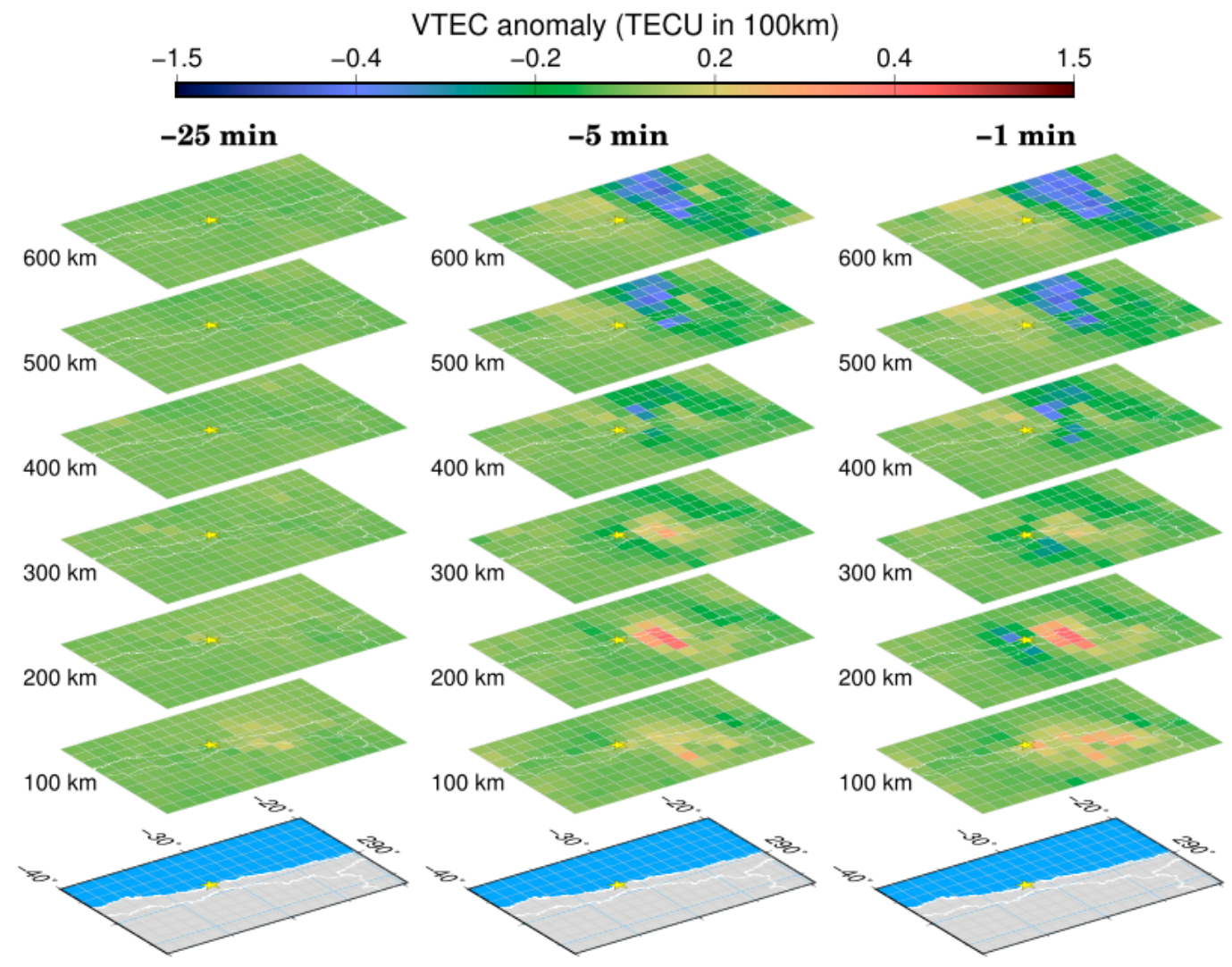

FIGURE 2.9: Electron density maps by height and time showing a wave travelling through the ionosphere before the 2015 Chilean earthquake struck [21]. 
recall a remark in the 1950s by the late J.A. Ratcliffe, a pioneer in F2-layer studies, to the effect that 'if you look for something in the F2-layer, you can always find it'" [1]. It is obvious that this comes as a double-edged sword; a correlation between disturbing events and ionospheric propagation must be proved, not merely observed, for fear of seeing that which does not truly exist. 


\section{Chapter 3}

\section{Methods}

\subsection{Motivation}

Once the local experiments were complete (described in Appendix B), the next experimental stage was proposed in collaboration with DRAO (Dominion Radio Astrophysical Observatory). The experiment was devised as a step towards the goal of imaging the ionosphere using two-dimensional arrays.

The researchers at DRAO were interested in the project for the following reasons:

1. To remove ionospheric disturbance from their astronomical data collections (as in adaptive optics) to reduce noise in their data.

2. To reuse a new piece of instrumentation developed by DRAO for a different experiment (discussed in Section 3.3.2).

3. To support RF research by a neighbouring university.

The first proposal happened in the fall of 2017 [23] and a second iteration was accepted by all parties in the summer of 2018 [24]. The experiment was conducted over a two-week period in late-June/early-July 2018.

The motivations for UViip to conduct this experiment at DRAO were as follows:

1. to strengthen collaboration between DRAO and UViip

2. to operate the experiment on a relatively 'radio-quiet' site

3. to take advantage of a temporarily available phase-coherent piece of instrumentation (discussed in Section 3.3.2)

4. to use an open field at DRAO which has a small portable room installed in the middle. The portable room has internet and power, meeting important constraints for the geographic specification for the experiment.

\subsection{Experimental preparation}

Before the experiment could be run, the instrumentation had to be built and tested to a variety of prearranged specifications. This substantial effort is outlined in Appendix A. 
TABLE 3.1: ENI 2100L RF Power Amplifier specifications [25].

\begin{tabular}{cc}
\hline \multicolumn{2}{c}{ Specifications } \\
\hline Start frequency & $10 \mathrm{kHz}$ \\
Stop frequency & $12 \mathrm{MHz}$ \\
Rated power & $100 \mathrm{~W}$ \\
Power gain & $50 \mathrm{~dB}$ \\
Connectors & $\mathrm{BNC}$ \\
\hline
\end{tabular}

\subsection{Experimental configuration}

The experiment was run between Penticton and DRAO, which created an approximately $20 \mathrm{~km}$ separation between transmitter and receiver. The receiver SDR used meant that continuous wave forms would be easier to analyze than the LFM sweeps transmitted in the local experiment (documented in Appendix B). This lead to a few new design objectives:

- It was preferable that the nulls of the transmitter and receiver antennas point at each other in order to eliminate ground wave interference with the ionospherically disturbed sky wave.

- There was no need for a reference wave since the waveform used was continuous.

\subsubsection{Transmitter}

Dr. Stephen Harrison kindly allowed a transmission antenna to be erected on his property in Penticton. A $40 \mathrm{~m}\left(\frac{1}{2} \lambda\right)$ dipole was raised with its center on Dr. Harrison's chimney and the ends tied to either a tree or a fence near the property edges. The property is $37 \mathrm{~m}$ long, which can be considered the effective length of the dipole. Where the antenna was longer than the property, the wire was wrapped along the fence or tree depending on which end of the property it was attached to. This made a roughly inverted- $V$ antenna shape which has a relatively good NVIS radiation pattern. The radiation pattern is the strongest orthogonal to the antenna wires so there is a null vertically and off to the sides of the antenna. Unfortunately, the shape of the property didn't allow the null of the antenna to point at DRAO.

The transmissions were amplified using an old Electronics \& Innovation 2100L RF Power Amplifier. It amplifies the input linearly as the amplitude of the input increases. Table 3.1 lists some amplifier specifications.

Initially a USRP N210 was used to generate the waveform but to simplify transmitting, a Tektronix AFG3252 Dual Channel Arbitrary Function Generator was used instead [26]. The function generator was synchronized with the receiving radio (Section 3.3.2) via a GPS frequency reference.

\subsubsection{Receiver}

The receiver end of the experiment was setup at DRAO. Here it is broken into two components for ease of understanding: the beamformer and the receiver array. 


\section{Beamformer}

The entire experiment was setup at DRAO because of the availability of a phasecoherent multi-channel beamformer built by Dr. Stephen Harrison and his colleagues at DRAO [27]. It was made as a demonstration that phased-array feeds can compete with traditional single-pixel feeds when used for post-correlation astronomical beamforming. Traditional single-pixel feeds have a radiation pattern based around a lone receiving sensor. A reflector will focus the radio waves of interest to this receiver which is then interpreted and turned into a "single-pixel" in a celestial image. In a phased-array feed, a multi-element phase-coherent sensor receives the focused radio waves from the reflector and each receiver element creates a "pixel" which can be used in an image. This also means that the radiation pattern can be changed by weighting each element of the phased-feed differently.

The beamformer was built to process up to 16 physical channels simultaneously. The beamformer is designed such that some wider effective bandwidth $\left(B W_{\text {eff }}\right)$ is needed and made up from $k$ frequency channels of channel bandwidth $B W_{e f f} / k$. From each physical channel (1-16), a single frequency channel is processed at once. $\mathbf{x}_{k}[n]$ is the vector of 16 antenna voltages from frequency channel $k$, at timestamp $n$.

For the beamforming, these sampled antenna voltages are multiplied with a complex weight vector $\mathbf{w}$

$$
b_{j k}[n]=\mathbf{w}_{j k}^{H} \mathbf{x}_{k}[n]
$$

in which $j$ is the beam number. This allows for an arbitrary number of beam directions to be processed simultaneously. Astronomers seem most interested in finding the power spectral density $(S)$ of each beam $(j)$. This is done by multiplying and averaging each weighted vector $(b)$, with its complex conjugate over an averaging period $(N)$

$$
S_{j k}=\frac{1}{N} \sum_{n=1}^{N} b_{j k}[n] b_{j k}^{*}[n]
$$

Understanding that the conjugate transpose of a vector is the same thing as the Hermitian of the vector, it follows from Dr. Harrison's paper that equation 3.2 can be expanded to

$$
S_{j k}=\frac{1}{N} \sum_{n=1}^{N} \mathbf{w}_{j k} \mathbf{x}_{k}[n] \mathbf{w}_{j k}^{H} \mathbf{x}_{k}^{H}[n]
$$

and Dr. Harrison further explains that if the weight vector is static or its change in time $\left(\frac{\delta \mathbf{w}}{\delta t}\right)$ is slower than the averaging period, $N$, that it can be further reduced to

$$
S_{j k}=\mathbf{w}_{j k} \hat{\mathbf{R}}_{k} \mathbf{w}_{j k}^{H}
$$

where $\hat{\mathbf{R}}_{k}$ is defined as

$$
\hat{\mathbf{R}}_{k}=\frac{1}{N} \sum_{n=1}^{N} \mathbf{x}_{k}[n] \mathbf{x}_{k}^{H}[n]
$$

The interest to UViip was not to do with the beamforming but with the fact that the device is phase-coherent. The output of the device is $\hat{\mathbf{R}}_{k}$ which is being called the array covariance matrix (ACM). 
TABLE 3.2: Beamformer parameters used during the DRAO-UViip experiment.

\begin{tabular}{cc}
\hline \multicolumn{2}{c}{ Parameters } \\
\hline Number of physical channels & 4 \\
Number of frequency channels $(k)$ & 512 \\
Effective bandwidth $\left(B W_{e f f}\right)$ & $3.584-4.096 \mathrm{MHz}$ \\
Frequency channel bandwidth & $1 \mathrm{kHz}$ \\
True sampling rate & $1.024 \mathrm{MHz}$ \\
Integration count $(N)$ & 400 \\
Integration time & $0.4 \mathrm{~s}$ \\
Effective sampling rate & $2.5 \mathrm{~Hz}$ \\
Nyquist zone & 8 \\
\hline
\end{tabular}

This output ACM is complex data which allows for the extraction of phase information.

The array covariance matrix is square and made up of both auto-correlation and cross-correlation components. The auto-correlation components are all along the diagonal and without a phase component while the cross-correlation components make up the upper triangle of the square. By taking the complex conjugate of the upper triangle, a lower triangle is created later. The number of auto-correlations is the number of antennas $\left(N_{\text {ant }}\right)$ while the number of cross-correlations is

$$
\frac{N_{\text {ant }}\left(N_{\text {ant }}-1\right)}{2}
$$

For a number of reasons discussed later in Section 3.3.2, the integration rate had to be lowered further than initially planned. Table 3.2 lists the parameters the beamformer used during the experiment at DRAO.

Note that the Nyquist zone is even resulting in a reversed channel/frequency relationship. As channels increase, frequencies decrease. This does not impact the running/setup of the experiment but when processing the information later must be (and is) taken into account.

\section{Receiver array}

Upon arriving at DRAO with the equipment (described in Appendix A), it quickly became obvious that the experiment was not going to run exactly as planned. It was unreasonable to move the antennas around the field without substantial difficulty.

When the semi-circle of antenna orientations (Figure A.6) is imposed on the available area (Figure A.5) it results in Figure 3.1b which shows that the antennas reach far past the fence and over the surrounding hills and facility space. Over the fence were herds of cattle which would potentially trample and break the coax. Also, initially setting up the antennas took far longer than expected as moving through the fields had to be done slowly and carefully to avoid startling rattlesnakes, there was old equipment scattered around the field and unwinding $800 \mathrm{~m}$ of coax was a gentle and labourious job. This resulted in a revised plan to constrain the antennas to the fenced area shown in Figure 3.1a.

The transmitter was located to the north and so the revised array configuration was chosen to be partially parallel and partially perpendicular to the direction of the incoming waveform. This resulted in the antenna orientation depicted in Figure 3.2. 


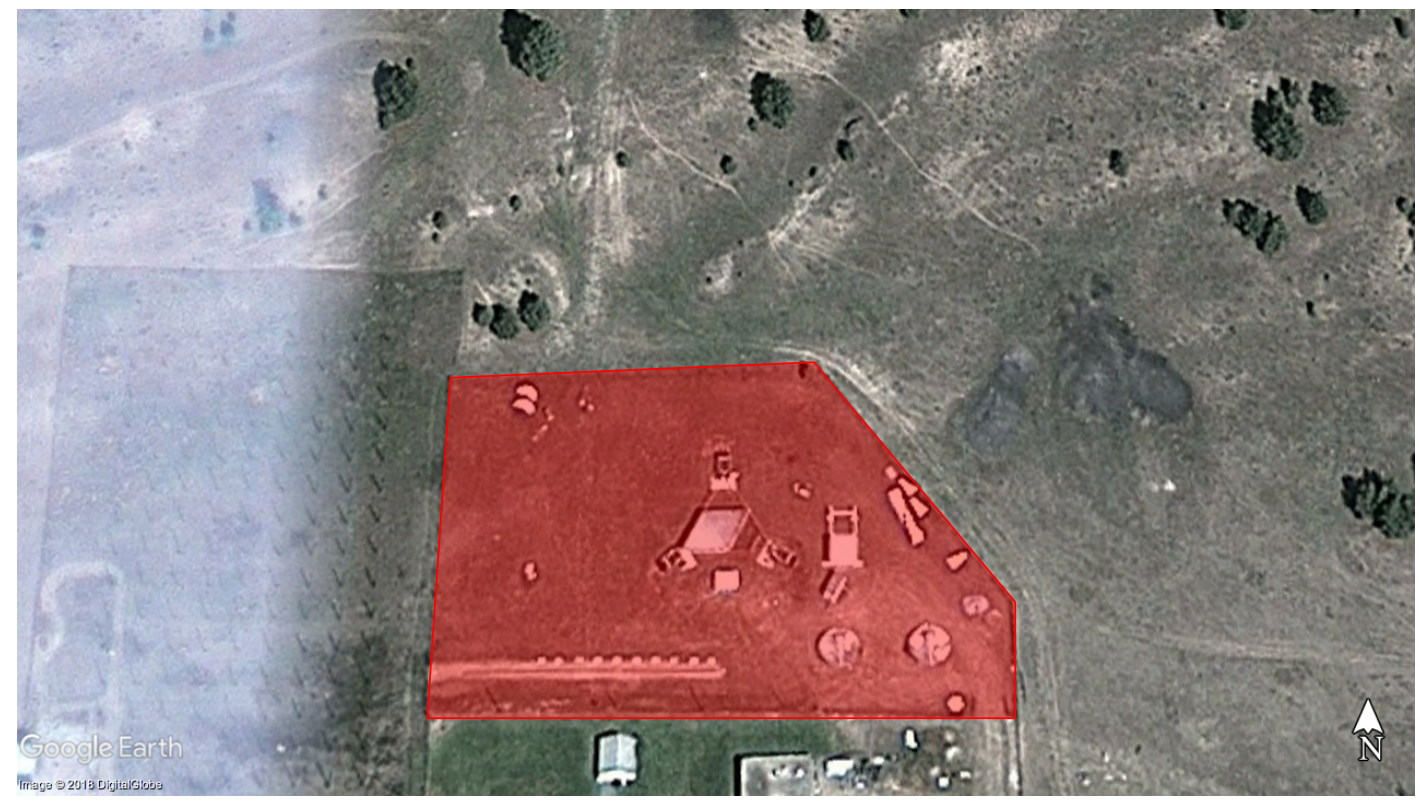

(a) Fenced HCTF area.

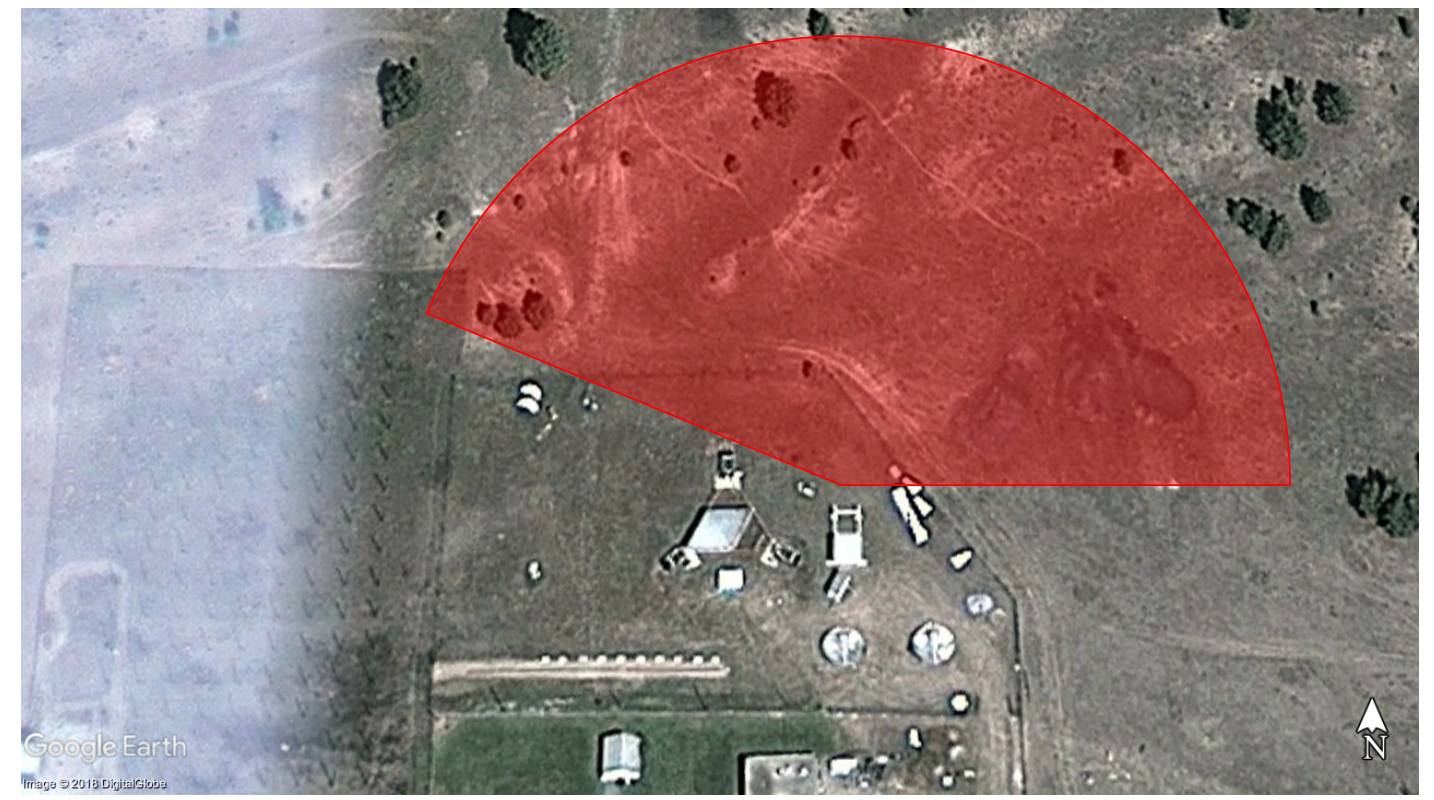

(b) Area the array circle would have covered.

FIGURE 3.1: Experimental areas at DRAO. 


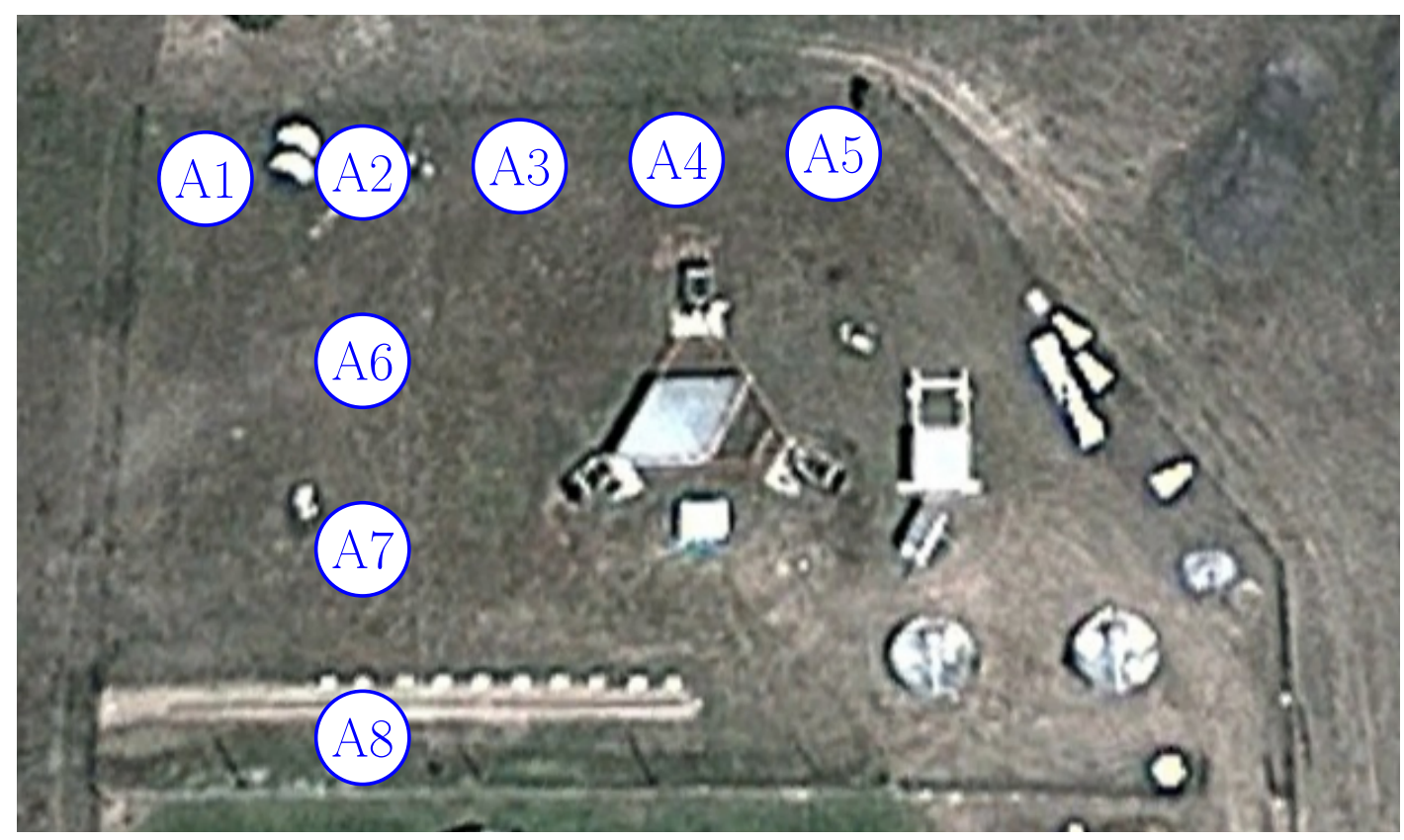

FIGURE 3.2: Orientation in which the antennas were setup.

A1-A5 provide an array of antennas perpendicular to the incoming waveform while A2-A8 provide an array that is parallel.

When the beamformer was first connected to the receiving array, all eight antennas were connected. It quickly became apparent that the line voltages were not above the minimum required by the ADCs so amplification was needed inline on each physical channel. The antenna voltage injectors were designed to provide sufficient signal (in the microvolt range) to a sensitive (noise figure of $10 \mathrm{~dB}$ or less) $\mathrm{HF}$ receiver. The ADCs in the beamformer were only sensitive to line level signals on the order of $1 \mathrm{~V}$.

Amplifiers were found on-site and although eight amplifiers (each providing 15 $\mathrm{dB}$ of gain) were found, they needed to be doubled up and soldered together to provide enough gain to make the ADCs usable. This decreased the effective number of antennas to four since each physical channel used two of the eight available amplifiers.

Aliasing was an issue since the beamformer does not have any analog or digital filtering available. $80 \mathrm{~m}(3.5-4 \mathrm{MHz})$ bandpass filters were purchased and shipped directly to DRAO. Figure 3.3 shows a block diagram of the system once setup. The filters were connected inline before the amplifiers because otherwise a signal from CKOR (a local $800 \mathrm{kHz}$ AM radio station operating with $10 \mathrm{~kW}$ which aliased into the frequency range of interest) was amplified and the ADCs were saturated.

Once everything was hooked up, the ADC voltages were more appropriate. Figure 3.4 shows that channels $0-3$ (out 16 beamformer inputs labelled 0 to 15 ) had more than the minimum required voltage while channels 4-7 did not. The histogram is probabilistic, so the y-axis is statistical frequency: the two 'flatter' curves, channels 1 and 2, see a wider range of available values and so are more sensitive. Channels 0 and 3 have a smaller range of values and so are less sensitive. Channels 4-7 do not have the minimum voltage required by the ADC (the entire frequency range exists at $0 \mathrm{~V}$ ) and is not sensitive at all. The $\mathrm{x}$-axis is reference voltage units which are proportional to the reference input.

At one point before the experiment began, strong winds blew over several of 
RX dipole

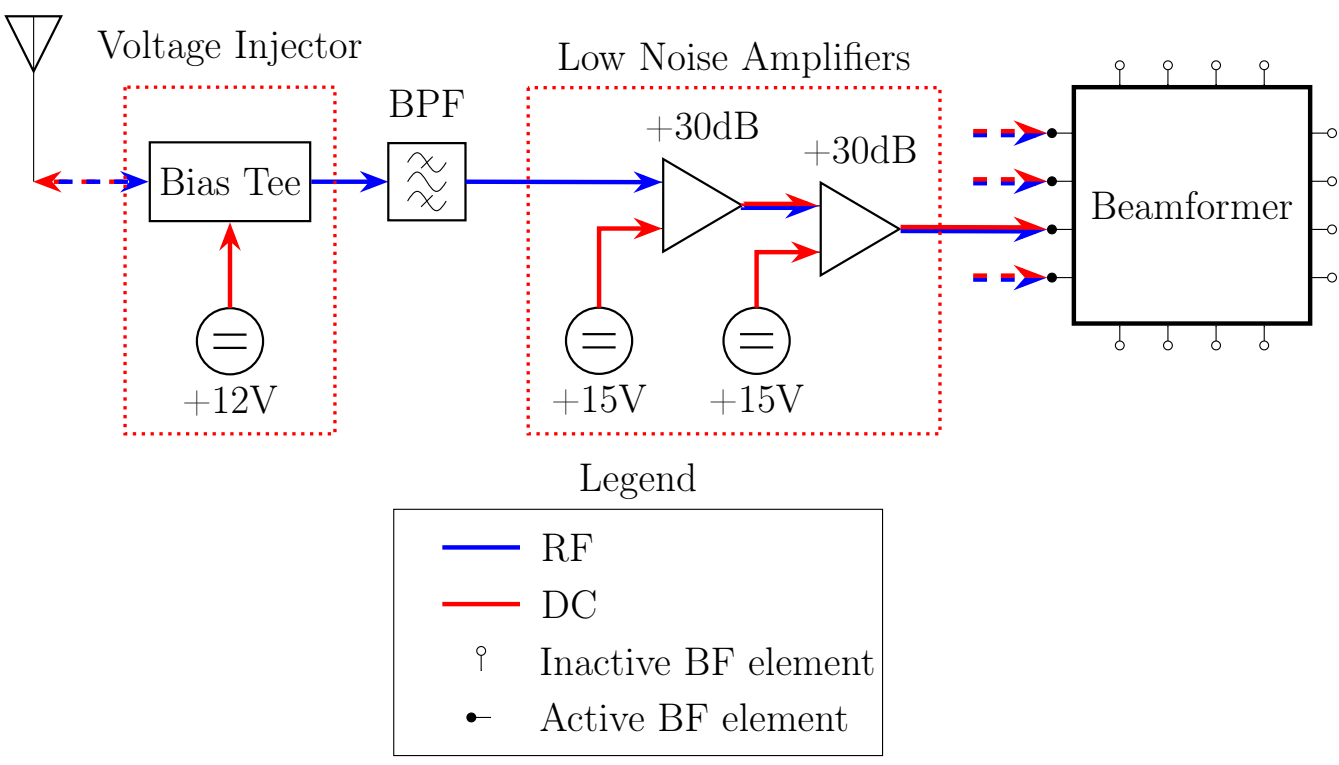

FIGURE 3.3: Block diagram of receiver system.

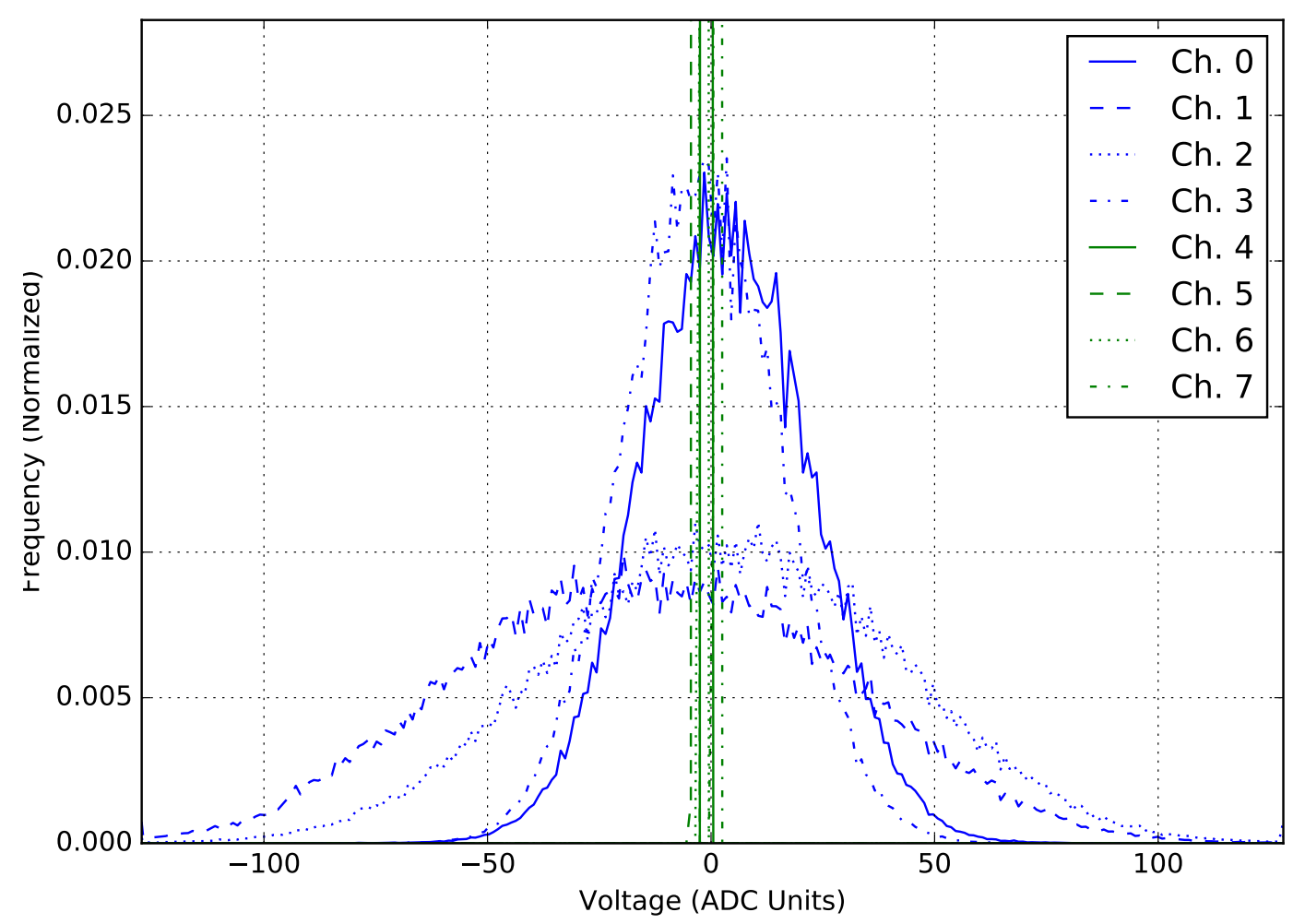

FIGURE 3.4: Histogram of ADC (10-bit) channel voltages. Blue curves are ADC channels of active inputs while green curves are channels which are are not attached to anything. 
TABLE 3.3: Antenna configurations.

\begin{tabular}{ccc}
\hline Configuration & Antennas & Recorded transmissions (hh:mm:ss) \\
\hline & & $3.7 \mathrm{MHz}$ CW $(00: 01: 02)$ \\
Config. 1 & $1 / 4 / 5 / 6$ & $3.7 \mathrm{MHz}$ CW $(00: 12: 10)$ \\
& & $3.8 \mathrm{MHz}$ CW $(00: 11: 24)$ \\
& & $3.7-3.706 \mathrm{MHz}$ LFM $\Delta t=0.4 \mathrm{~s}(00: 33: 22)$ \\
\hline & & $3.7 \mathrm{MHz}$ CW $(00: 33: 22)$ \\
Config. 2 & $2 / 6 / 7 / 8$ & $3.8 \mathrm{MHz}$ CW $(00: 08: 03)$ \\
& & $3.720-3.726 \mathrm{MHz}$ LFM $\Delta t=0.4 s(00: 33: 23)$ \\
\hline
\end{tabular}

the antennas. Considering the antenna labels from Figure 3.2, antenna 7 blew over with the antenna pre-amplifier box facing upwards, while antenna 3 blew over with the antenna pre-amplifier box facing downwards. The only apparent damage on antenna 7 was a bent tip on one of the whips while on antenna 3 the front panel of the pre-amplifier box was crushed inwards. To stop this from happening again, the tripods were lowered by four feet. The antennas were now all 16 feet high instead of the manufacturer recommended 20 feet. At this point the experiment was ready to be run.

\subsection{Running the experiment}

Due to the geographic separation between transmitter and receiver, the experiment could not be easily changed without driving between Penticton and DRAO. The antennas of interest were connected to the beamformer (four at a time) at DRAO, while the transmitter had to be powered up from Dr. Harrison's house in Penticton. There needed to be personnel at DRAO to change antennas and personnel in Penticton to operate the transmitter. DRAO is a radio-quiet site and so it wasn't possible to have people at each location communicating over the phone. Instead, a single antenna configuration was used each day and after it was setup, the beamformer was remotely managed to start and stop the recordings from Penticton.

Two antenna configurations were chosen: one perpendicular to the direction of transmission and one parallel to the direction of transmission. Table 3.3 shows the antenna numbers used and the transmissions done with each configuration. Figures $3.5 \mathrm{a}$ and $3.5 \mathrm{~b}$ show the two array configurations on a map of the area. Two antenna configurations were chosen: one perpendicular to the direction of transmission and one parallel to the direction of transmission. Table 3.3 shows the antenna numbers used and the transmissions done with each configuration. Figures $3.5 \mathrm{a}$ and $3.5 \mathrm{~b}$ show the two array configurations on a map of the area. 


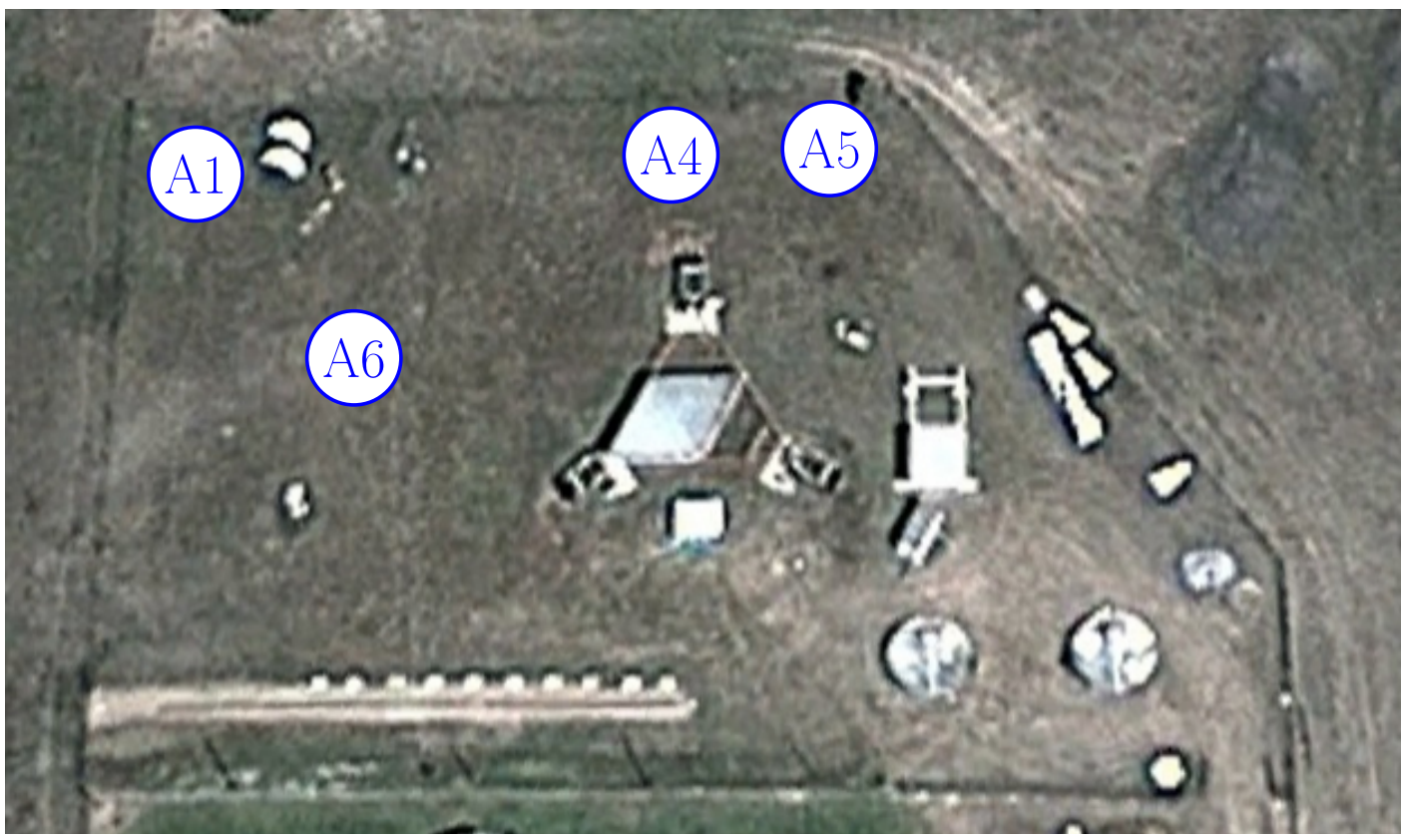

(a) Configuration 1.

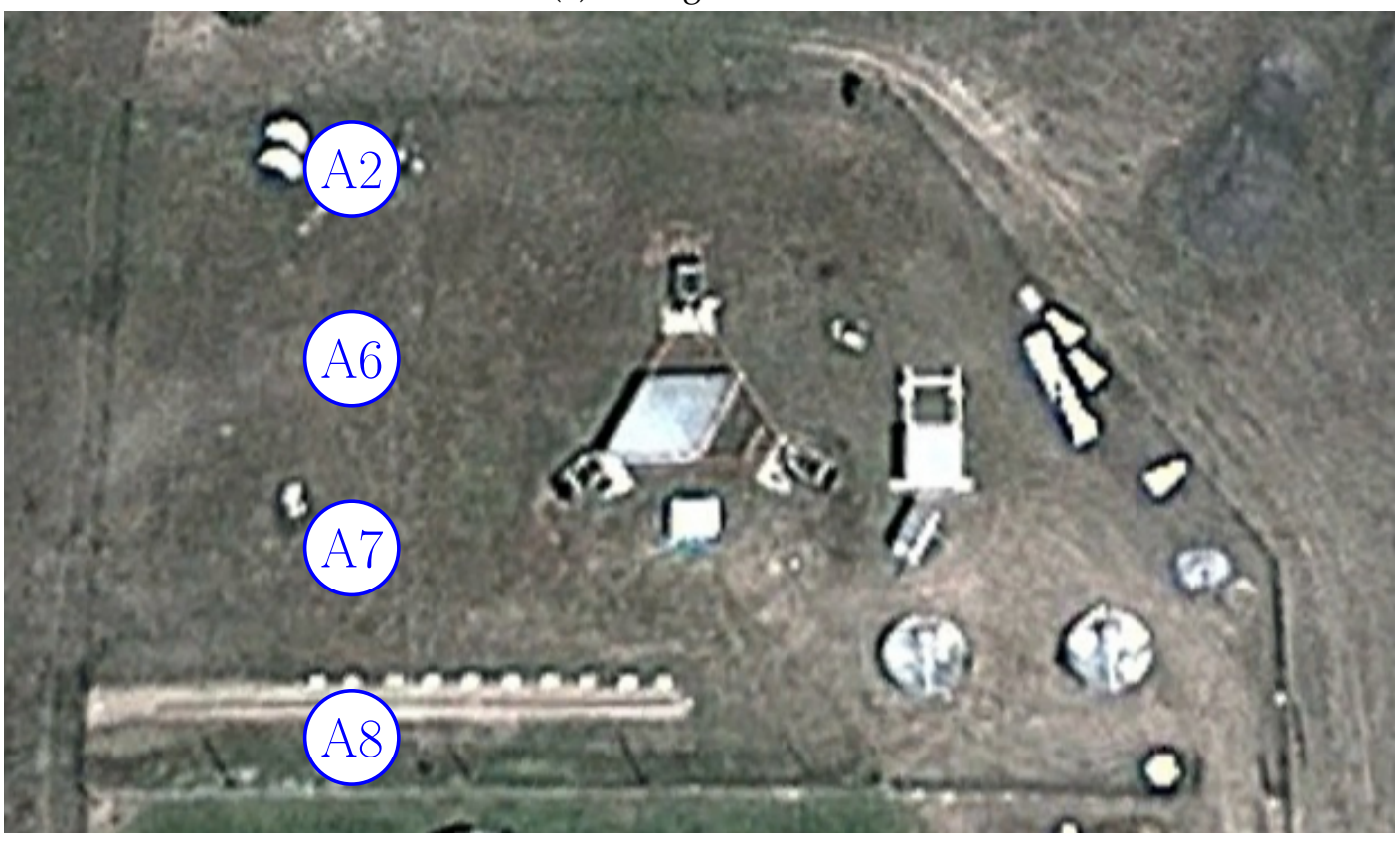

(b) Configuration 2.

FIGURE 3.5: Final array configurations of antennas, see Table 3.3. 


\section{Chapter 4}

\section{Results and Discussion}

\subsection{Visualizing data during collection}

While the experiment was being run, it was visualized in a few different ways. This section (4.1) shows the way the data was visualized while it was being collected, while later (Section 4.2) the processed data is discussed. Each time data was collected (transmission was occurring while the receiver was recording), it is referred to as a session while the overall effort is referred to as the experiment.

One way in which the data was visualized during the sessions is shown in Figure 4.1. This is an average of the ACM, which updates every $0.4 \mathrm{~s}$ (see Table 3.2). Configuration 1 (Figure 4.1a) is shown with values under antenna indexes $1,4,5$, 6, while configuration 2 (Figure 4.1b) has grid values for antenna indexes 2, 6, 7, 8. Figure 4.2 is shown again here for convenience in interpreting the ACM indexes. Remembering that each of the 10 correlations has a bandwidth of $512 \mathrm{kHz}$, these grids take the magnitude and phase from each of the 512 channels and average them together. The lower triangles were calculated as the complex conjugate of the upper triangles.

Note that the auto-correlations (diagonals) are zero on the phase averages since there is no phase component to the auto-correlation. Note as well that the magnitude was not measured relative to anything in particular and are in arbitrary units (in this case, arbitrary $\mathrm{dB}$ ). This is not problematic because the phase is the value of interest, and is relative between antennas.

To check what the channel is receiving, each grid index can be unpacked into a magnitude and phase spectrum and waterfall as shown in Figure 4.3. Like the grid of averages, the spectrum and waterfall each update every $0.4 \mathrm{~s}$. The spectrum shows in 2D what the current 3.5-4 MHz range looks like, while the waterfall is $3 \mathrm{D}$, representing each of the previous spectra as a single line of width $0.4 \mathrm{~s}$ where the color of each pixel indicates its magnitude (or phase).

Compared to normal waterfall plots, where the plot is signal strength relative to $0 \mathrm{~Hz}$ (DC), it's important to distinguish that these spectra are relative between two antennas. In the case of Figure 4.3, the spectra are relative between antennas 1 and 5 (and so from configuration 1).

\subsubsection{Radio frequency interference}

Despite DRAO being a relatively noise-free environment at the higher-frequencies used for radio astronomy, at the lower frequencies used for this experiment, there was some RFI (radio frequency interference). Apparent in Figure 4.3 is the very strong 3.8 MHz signal transmitted for this session. Only the data in the $1 \mathrm{kHz}$ channel surrounding the transmitted frequency is used while analyzing the $\mathrm{CW}$ data sets (Section 4.2), and so RFI in other frequency channels doesn't impact the quality of 


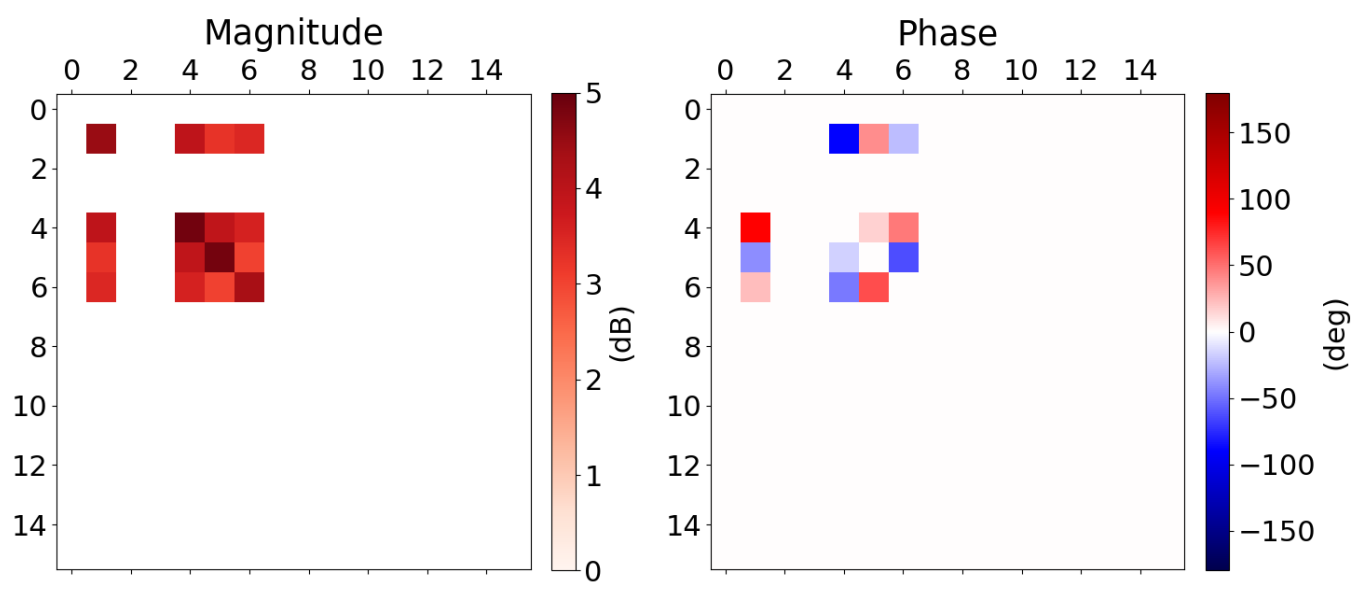

(a) Configuration 1.

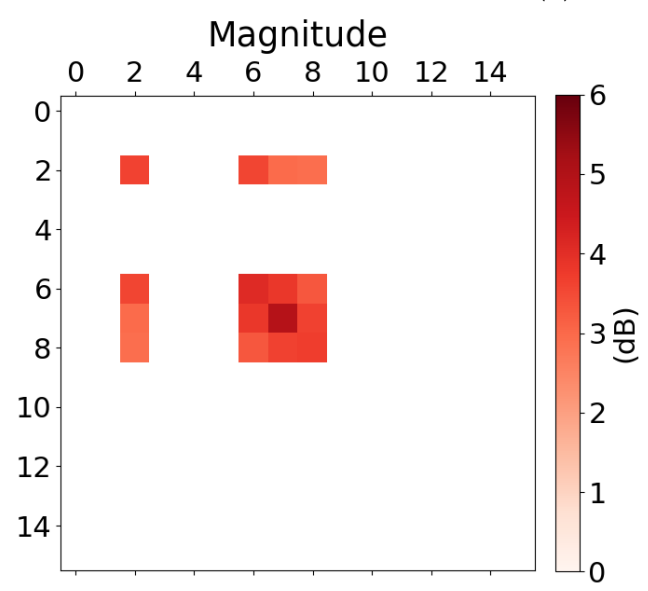

Phase

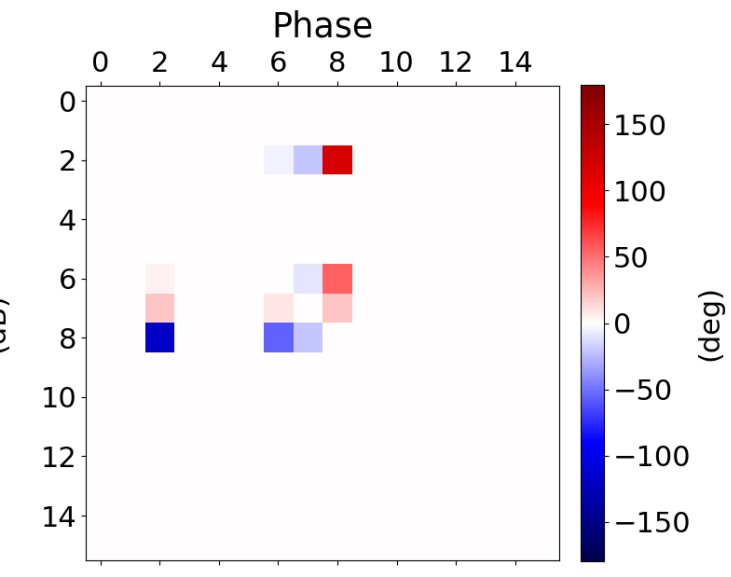

(b) Configuration 2.

FIGURE 4.1: Average magnitude and phase of the ACM. White indicates no data or 0 as is the case for all of the diagonal elements of the phase (auto-correlation has no phase). Where the indices are the same, an autocorrelation was done, where they differ, a crosscorrelation was done. The index shows the antenna element (see Figure 3.5). Each plot represents a $0.4 \mathrm{~s}$ snapshot of the data.

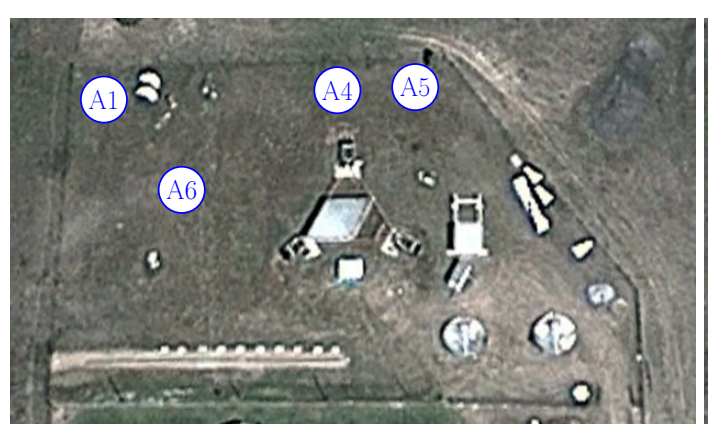

(a) Configuration 1.

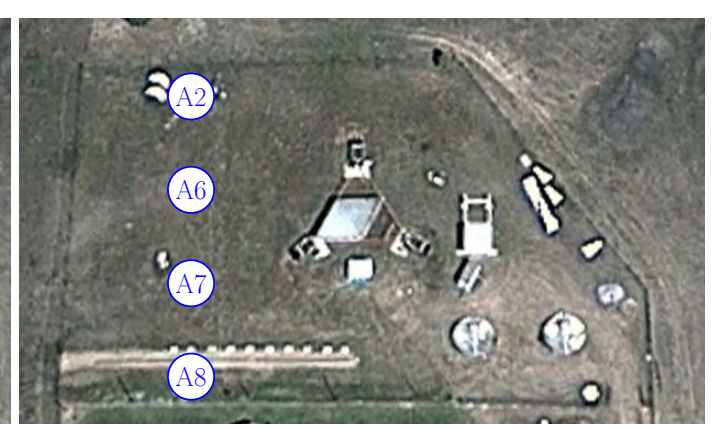

(b) Configuration 2.

FIGURE 4.2: Array configurations, (originally shown in Figure 3.5) shown here for convenience in interpreting Figure 4.1. 


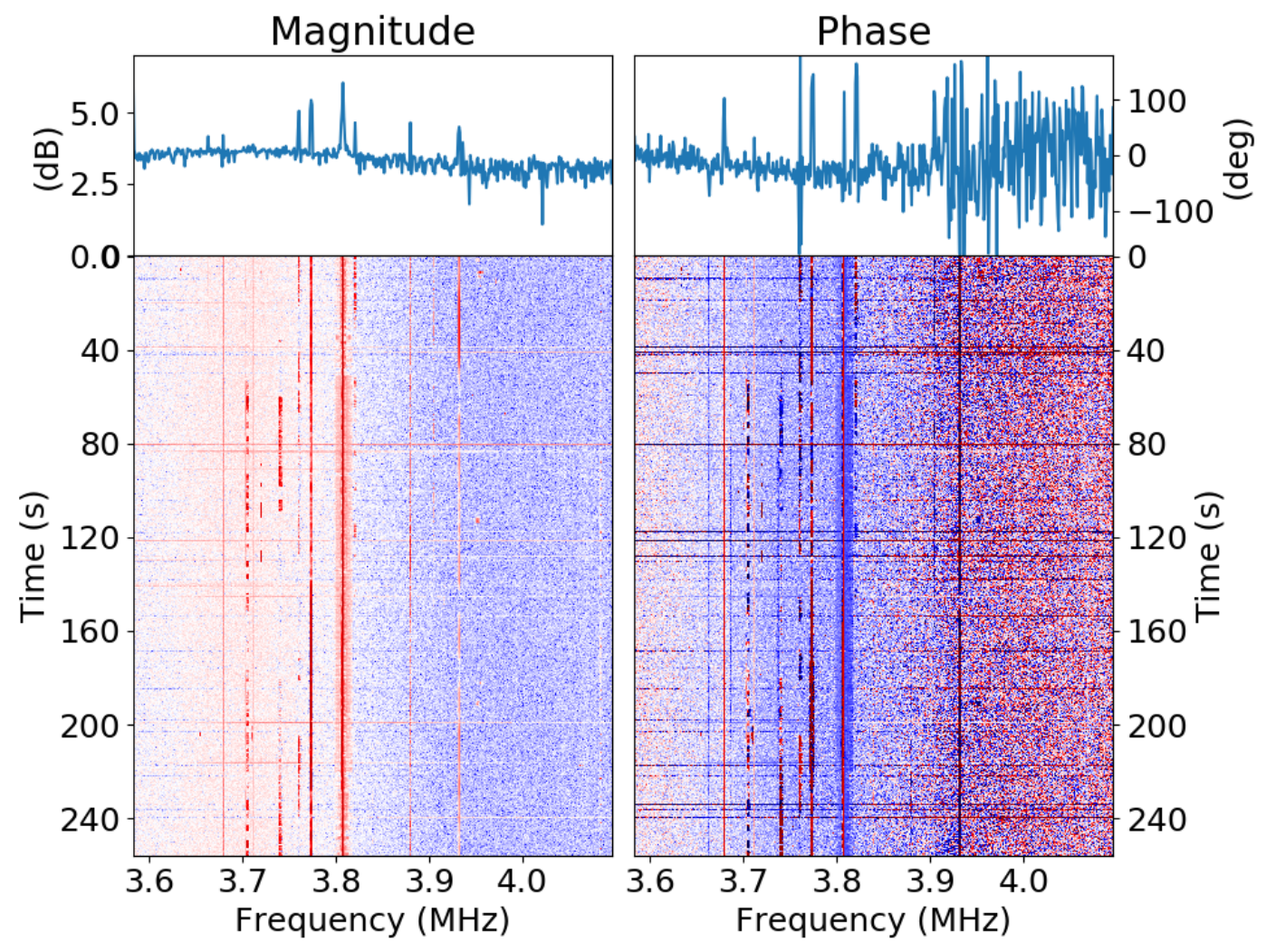

FIGURE 4.3: Relative magnitude and phase between antenna 1 and antenna 5. Note that the phase appears more coherent at lower frequencies. 

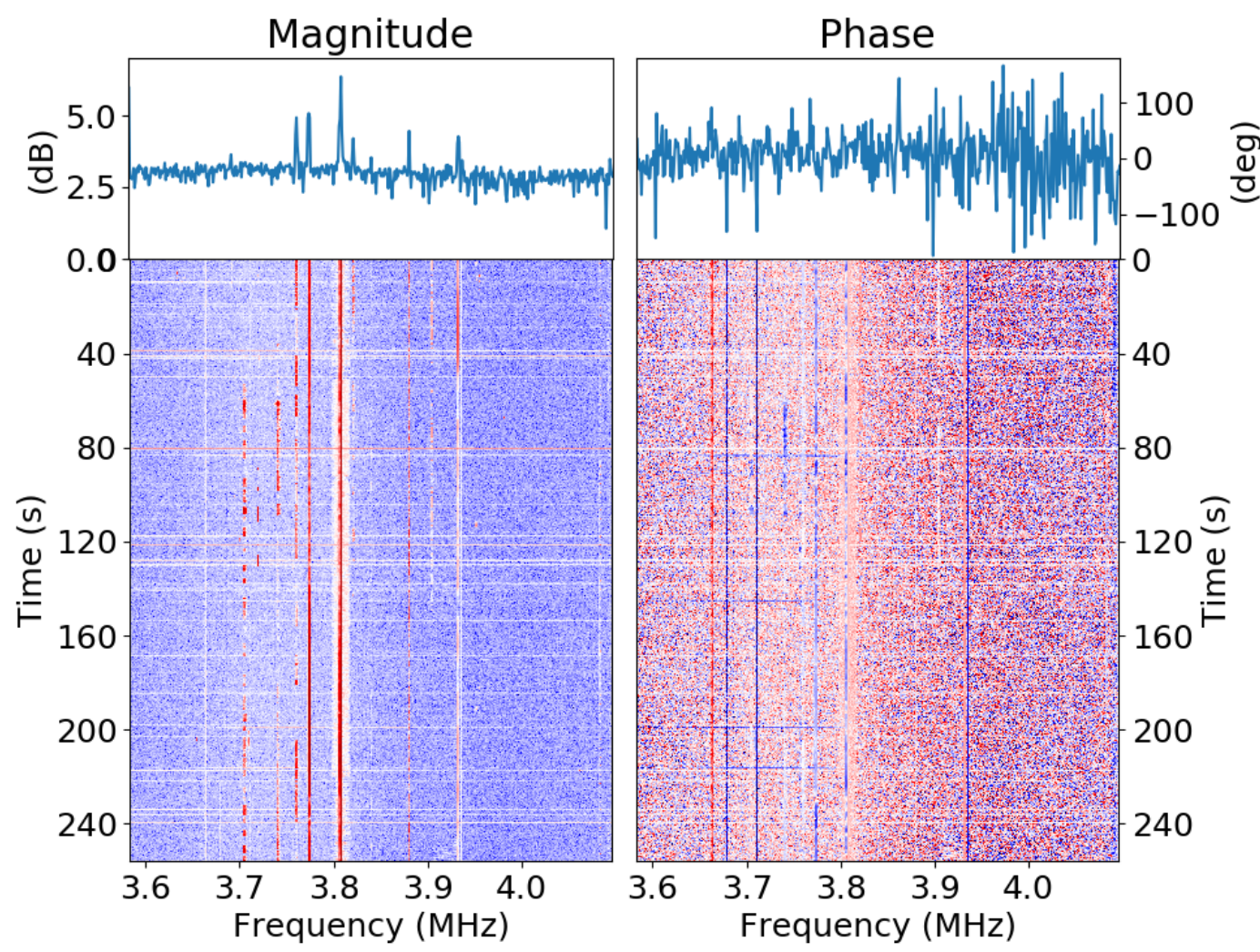

FIGURE 4.4: Relative magnitude and phase between antenna 1 and antenna 6. Note that the phase does not appear as coherent at lower frequencies as it did in Figure 4.3.

the collected data. The $800 \mathrm{kHz}$ AM station alias mentioned in Chapter 3 can also been seen.

\subsubsection{Spatial and frequency coherence}

Figure 4.3 shows a coherence at lower frequencies in phase and possibly magnitude. The waterfall plots show this observation is consistent throughout the experiment. To check whether this was true for all antenna pairings, other ACM grid indexes can be unpacked. Figure 4.4 shows the exact same time period as Figure 4.3, however the frequency-dependent coherence is not as apparent.

While looking into possible causes of this, it was found that there is a strong relative spatial coherence between the antennas as well. Figure 4.5 shows the consistent levels of coherence associated with the antenna spacing on the ground. This is possibly caused by various noise sources coming from DRAO. In this antenna configuration, the antennas used for the correlation in Figure 4.5 were organized perpendicular to DRAO.

\subsection{Visualizing data after collection}

Although multiple transmissions were recorded in each array configuration (in both CW mode and LFM mode, as outlined in Table 3.3) only the CW data has been analyzed. The CW data is relatively easy to understand considering there should only be one ionospheric reflection height for each transmitted frequency. Appendix B 


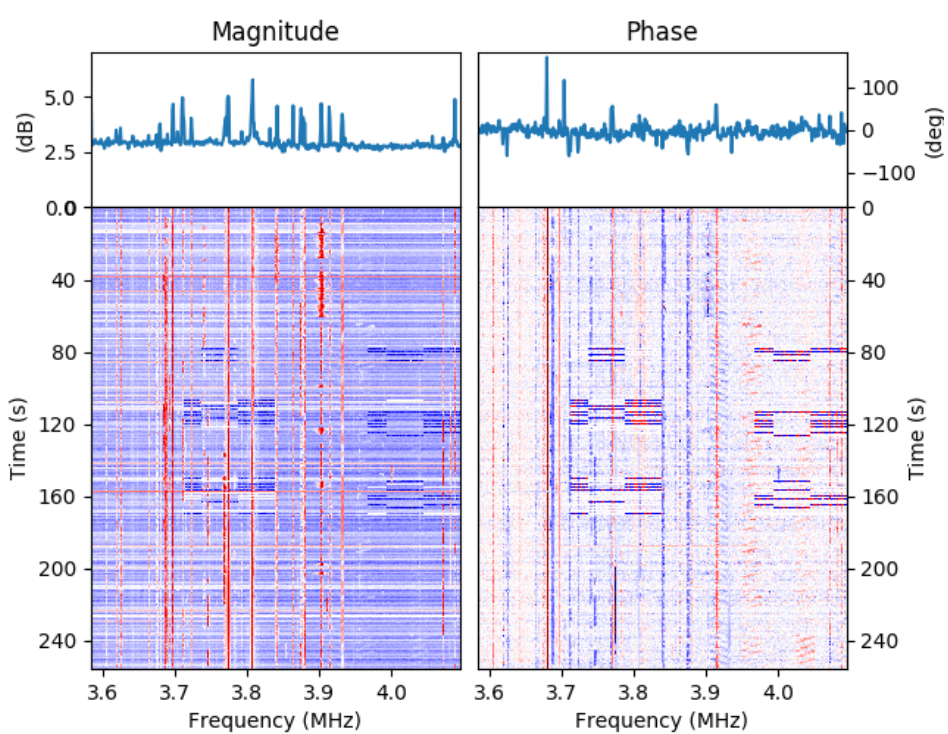

(a) $20 \mathrm{~m}$ spacing showing a high overall coherence between the antennas. Even througout the frequency ranges not receiving signals.

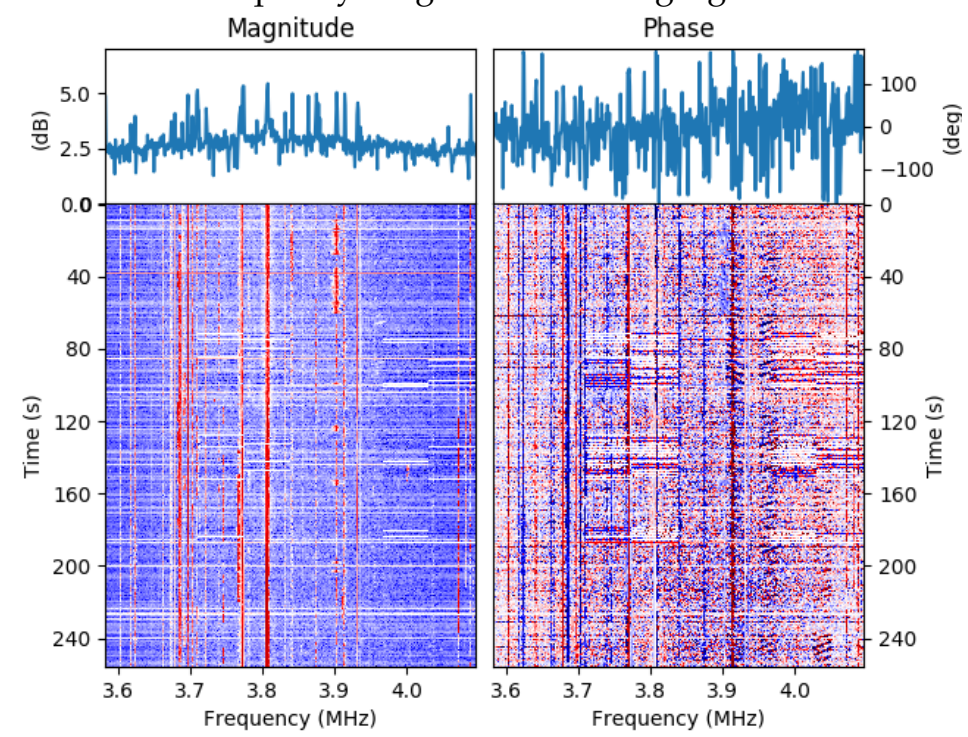

(b) $40 \mathrm{~m}$ spacing showing less coherence between the antennas than the $20 \mathrm{~m}$ spacing does.

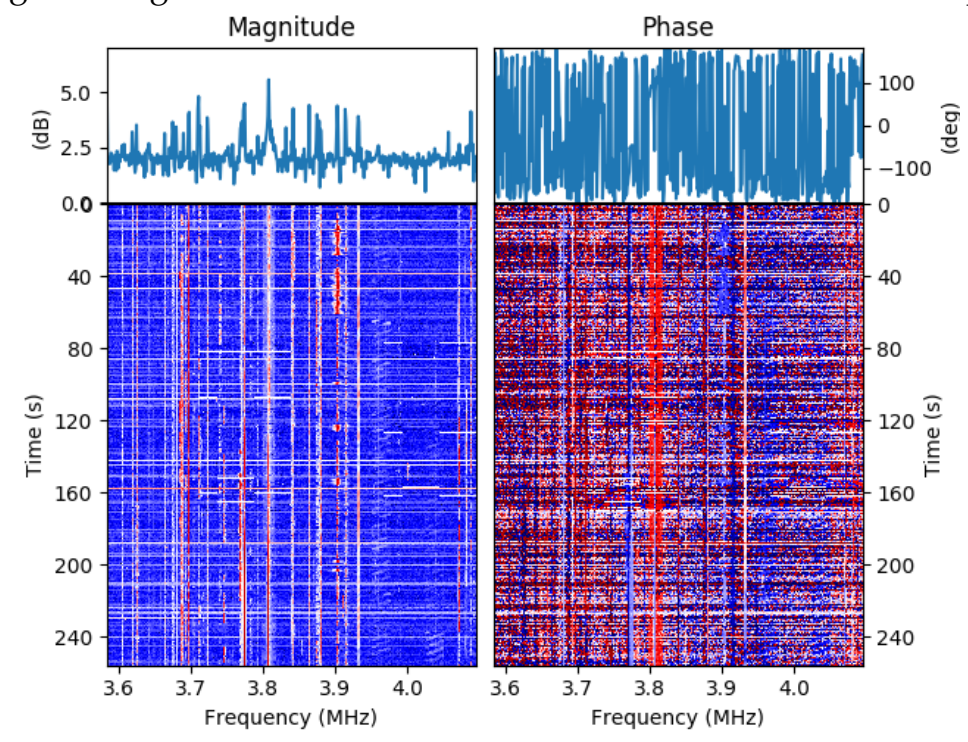

(c) $60 \mathrm{~m}$ spacing showing no coherence between the antennas where there is no received signal.

FIGURE 4.5: Antennas from configuration 2 but with different ground spacings. This shows a strong relative spatial coherence between closer antennas. 
discusses an earlier experiment done at UVic which only used LFM transmissions and experienced problems with phase-coherence likely attributable to the spreading of the signal through the ionosphere. It is highly recommended to further analyze these data sets.

\subsubsection{Single frequency time domain}

Knowing that the session from Figures 4.1 and 4.3 was at $3.8 \mathrm{MHz}$, it is possible to pick out the spike in the spectra at $3.8 \mathrm{MHz}$ and see the time-history of the $3.8 \mathrm{MHz}$ signal strength in the waterfalls. The $1 \mathrm{kHz}$ wide channel centered at $3.8 \mathrm{MHz}$ can be extracted and plotted in time which is shown in Figures 4.6a(the entire session) and $4.6 \mathrm{~b}$ (a zoomed in segment).

\subsubsection{Interpolating over missing data}

Note in the waterfalls of Figure 4.3 that some horizontal bars appear semi-regularly throughout the data. These are missing data points where the beamformer did not collect actual data and filled those spaces with zeros.

In order to manage those missing data points, software was written to linearly interpolate (in the time direction) over those points.

\subsubsection{Considering average phase differences}

Taking a very simple geometry like that in configuration 2 , it is relatively easy to predict how the data should look if the transmission was not perturbed before reception. The relative phase differences between antennas should grow as the physical distance between antennas grows, assuming that the antennas spacing is less than a wavelength. Configuration 2 is easier to visualize than configuration 1 since the array is aligned with the direction of transmission and so the transmitted radio wave had to travel further in a line to get to each antenna.

To check that the data supports this, a large rolling average is calculated. This rolling average (or moving average) is a central moving average as described in Equation 4.1. Here, $x_{i}$ is the $i^{\text {th }}$ element of the input array, $L$ is the window length, $N$ is the length of the input array, and $\bar{x}_{a v g}$ is the output array of length $N-L$.

$$
\bar{x}_{a v g}=\left.\frac{1}{L} \sum_{n=0}^{L} x_{i}\right|_{0} ^{N}
$$

Figure 4.7 shows the result. As expected, the relative phase between antennas 2 and 8 (ant28) is the largest separation on the ground $(\tilde{6} 0 \mathrm{~m})$ and sees what is consistently the largest phase difference. The smaller spacings (ant26, ant67, and78) are all 20m separated and see smaller phase differences. The medium separations of $\tilde{4} 0 \mathrm{~m}$ (ant27, ant68) are less obvious. Antennas 2-7 show a strong relation as expected while the 6-8 relation does not stand out from the smaller spacings. This could imply a phase mismatch somewhere in the physical system, or it could imply some sort of ionospheric disturbance.

\subsubsection{Mesh of ionospheric surface}

Taking a single antenna in a linear array as reference, another antenna has a phase difference relative to that reference. Knowing the physical spacing of those antennas 

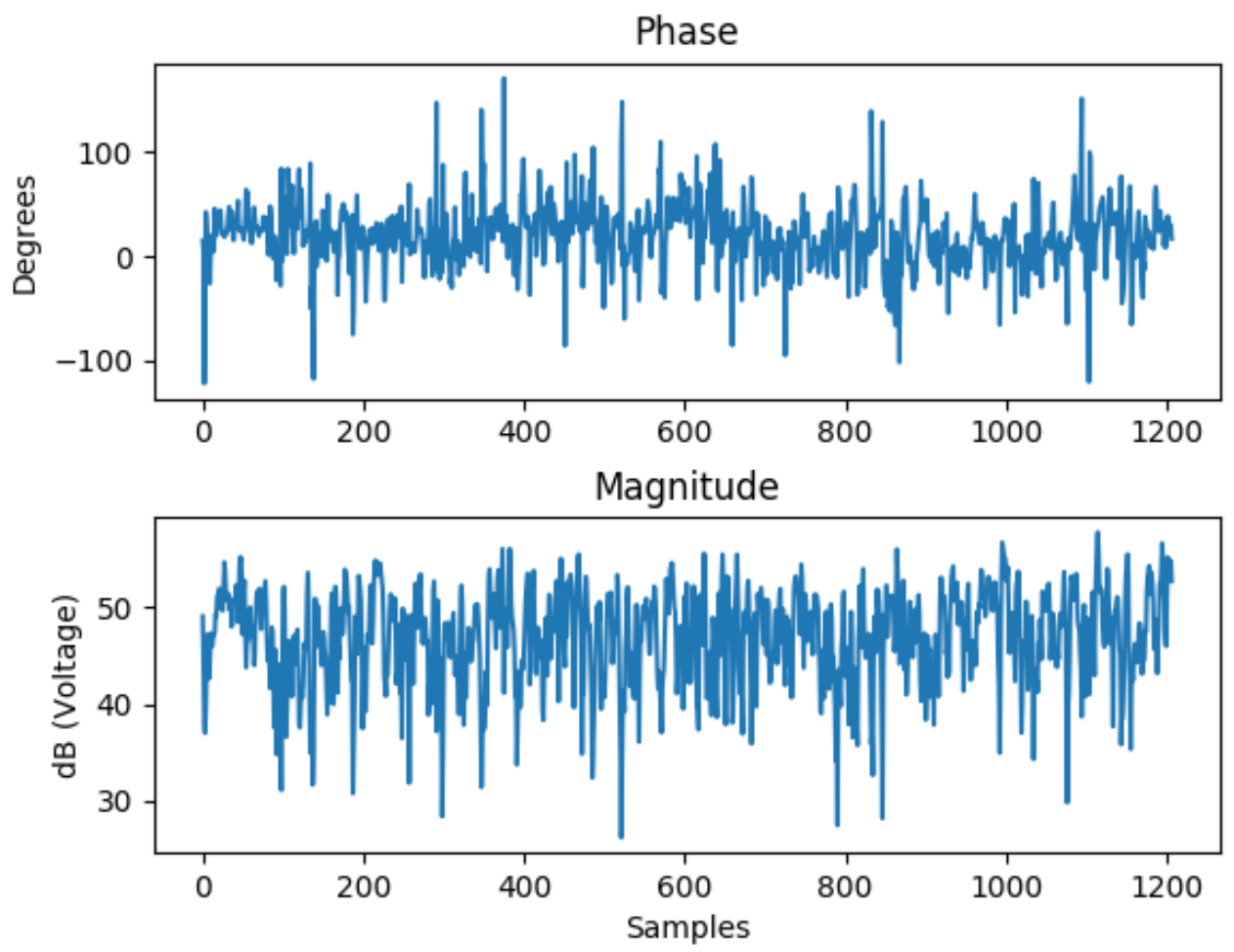

(a) Time domain of antennas 2 and 7 at $3.8 \mathrm{MHz}$ (full session).

Phase
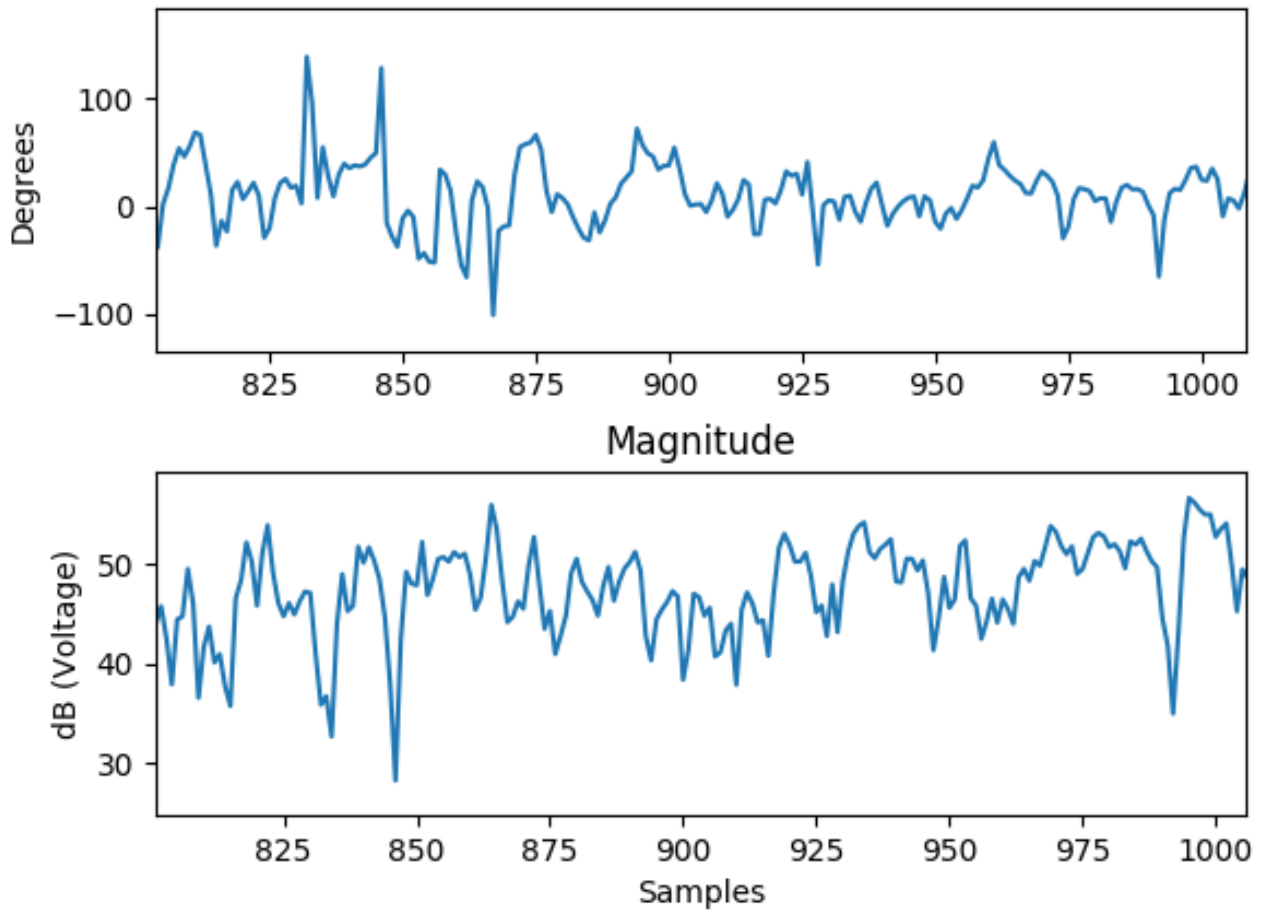

(b) Time domain of antennas 2 and 7 at $3.8 \mathrm{MHz}$ (zoomed in).

FIGURE 4.6: Time domain of $1 \mathrm{kHz}$ wide channel centered on the transmission frequency. 


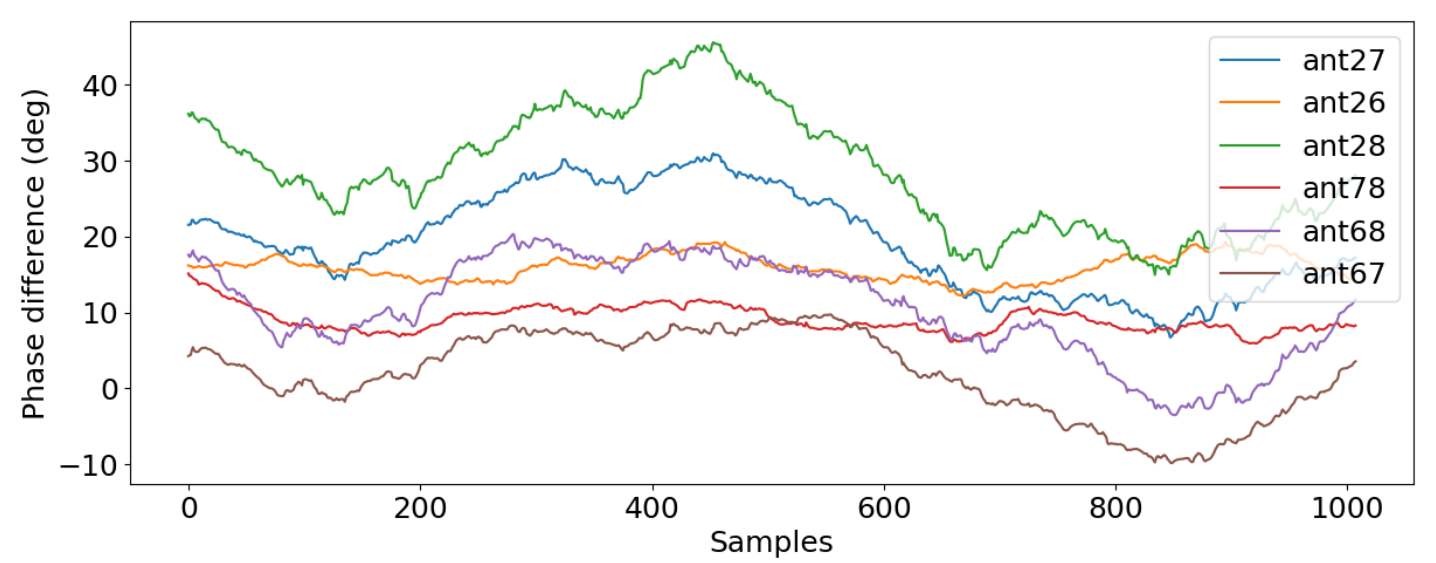

FIGURE 4.7: Rolling average of window length 200 samples, for a 3.8 $\mathrm{MHz}$ transmission.

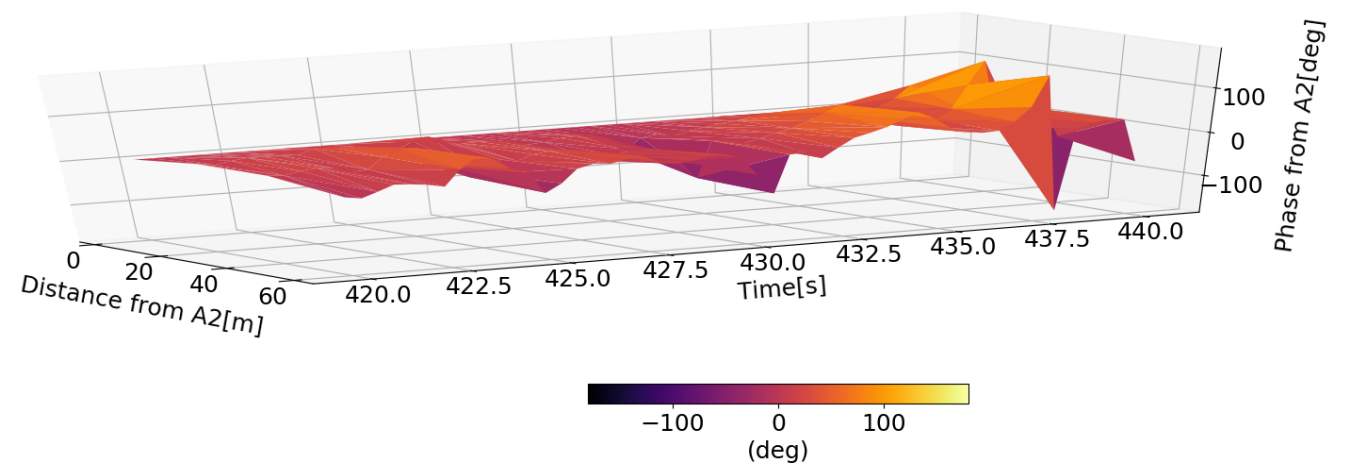

FIGURE 4.8: Mesh (surface) of 3.8 MHz signal received across phased array.

means that we can plot the phase differences changing over time against the antenna distance differences.

Since there are four antennas, there are three measurements relative to any reference. Taking configuration 2 as an example, and antenna 2 as a reference, there are phase differences available from antennas 2-6, 2-7, 2-8. These three phase differences can be meshed together into a single surface, which should represent an expanded (since the receiver is twice as far from the transmitter as the ionosphere) version of the ionospheric surface. Figure 4.8 shows a segment of the $3.8 \mathrm{MHz}$ data collected in configuration 2 . Note in the figure that distance 0 is kept as a reference since a phase difference doesn't exist there.

It is clear that there are fluctuations in the data consistent across the array. At approximately $437.5 \mathrm{~s}$ into the data there is a sharp peak which is consistent in the $2-6,2-7$, and 2-8 relative phase data. It is reasonable to attribute this to an increase in the height of the ionosphere. Similarly, at $430 \mathrm{~s}$ there is a decrease in relative phase indicating a decrease in ionospheric height.

A series of these images (frames) were combined to animate the ionospheric surface movement. Figure 4.9 shows several of these frames, each of which is a snapshot of the ionospheric surface over some time period. When all played together, the animation shows a peak travelling through the receiver array. It is clear from them that the peaks and troughs are consistent across the antenna array. When there is a small trough in the 2-6 data there is often a slightly larger one in the 2-7 data and an even larger one in the 2-8 data. The difference in the size of the trough is attributable to 
the spacings of the antennas (smaller spacings leading to smaller troughs) as well as implies that the cause of the trough is not moving entirely perpendicular to the antenna array.

\subsubsection{Frequency domain analysis}

Taking each array's relative phase time series data, an FFT was computed in the hopes of finding consistent frequency components. If found, the frequency of the ionospheric waves would be known. Figure 4.10 shows the results for each data array. As is shown, there are no obvious frequency components rising above the noise floor. This could be for a number of reasons but most likely a function of the low sampling rate $(2.5 \mathrm{~Hz})$. Although the team is still hopeful to extract some meaningful data on ionospheric wave movements, this process will be greatly eased by increasing the sampling rate during future experiments.

\subsubsection{Virtual height}

One interesting application of this project is to directly measure ionospheric characteristics like free-electron density. As discussed in Chapter 2, ionospheric height is proportional to electron density

$$
\phi_{m}=\frac{\lambda}{\left(\frac{\phi_{\text {rad }}}{\pi}\right)}
$$

The ionospheric height was calculated using a planar wave assumption. As shown in Figure 4.11, if the wavefront is assumed to arrive at the receiving array as a flat wave-front, the ionospheric height can be estimated. Equation 4.2 is used to calculate the distance $\left(\phi_{m}\right)$ proportional to the measured phase difference $\left(\phi_{\mathrm{rad}}\right) . \lambda$ is the wavelength of the transmitted frequency.

Equation 4.3 is used to find the ionospheric virtual reflection height using $\phi_{m}$ as well as $b$ (the distance between receiving antennas), $x_{1}$ and $x_{2}$ (the distances from the transmitter to each receiver antenna respectively). $b, x_{1}$ and $x_{2}$ were measured during the experiment. Note that if $\phi_{\text {rad }}$ (and $\phi_{m}$ by extension) is longer than a half wavelength, the triangle cannot be measured.

$$
\begin{aligned}
\theta & =\arccos \left(\frac{\phi_{m}}{b}\right) \\
h_{1} & =\tan \left(\theta \frac{x_{1}}{2}\right) \\
h_{2} & =\tan \left(\theta \frac{x_{2}}{2}\right) \\
h & =\frac{\left|h_{1}+h_{2}\right|}{2}+\min \left(h_{1}, h_{2}\right)
\end{aligned}
$$

Figure 4.12 shows the relationship between a phase difference measured between two antennas and the calculated ionospheric height. The equations relevance deteriorates as $\phi$ approaches either $\frac{\pi}{2}$ or 0 ( $\frac{\pi}{2}$ being the half-wavelength detection limit).

Figure 4.13 shows the results of this for the approximately 33 minute long configuration 2 session done at $3.7 \mathrm{MHz}$ (see Table 3.3). It is apparent in the image that each of the antenna pairings sees similar shifts but are not closely aligned otherwise.

Another way of calculating ionospheric height was considered which involves iterating from a planar wavefront into a spherical wavefront and using the difference 

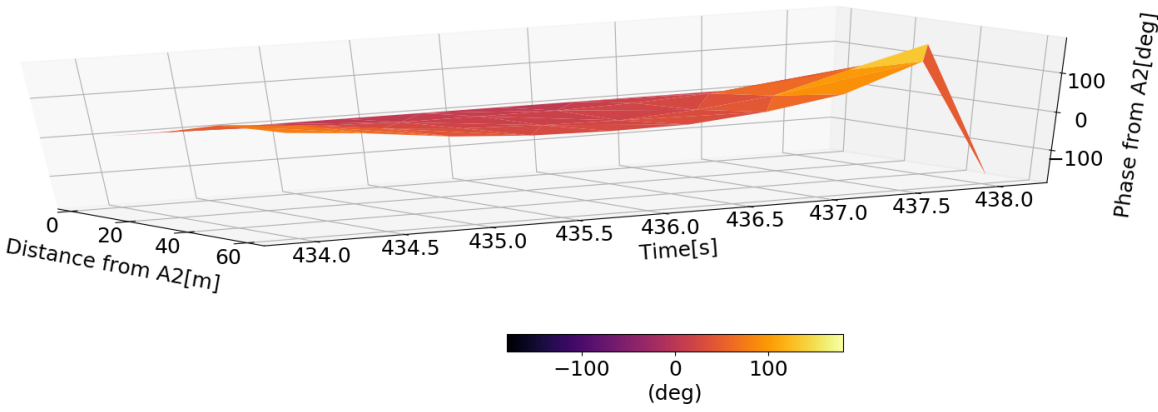

(a)

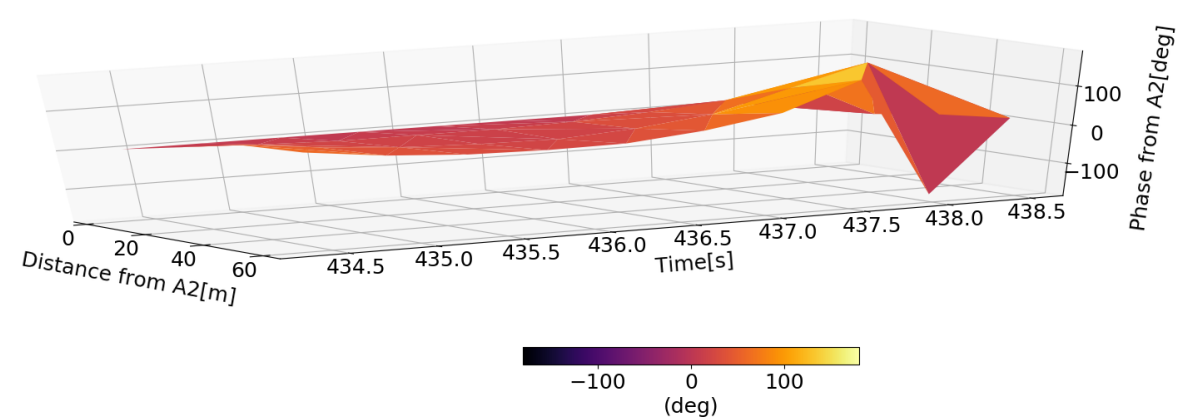

(b)

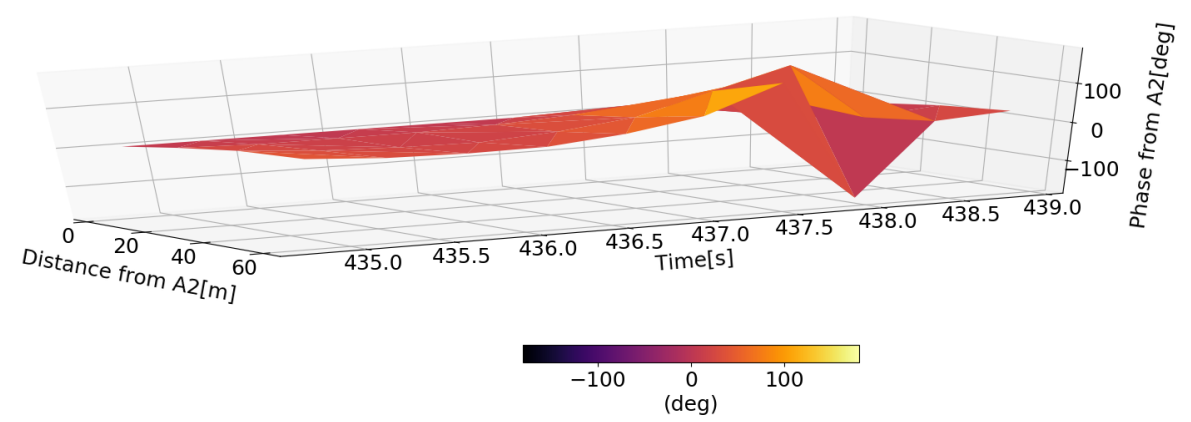

(c)

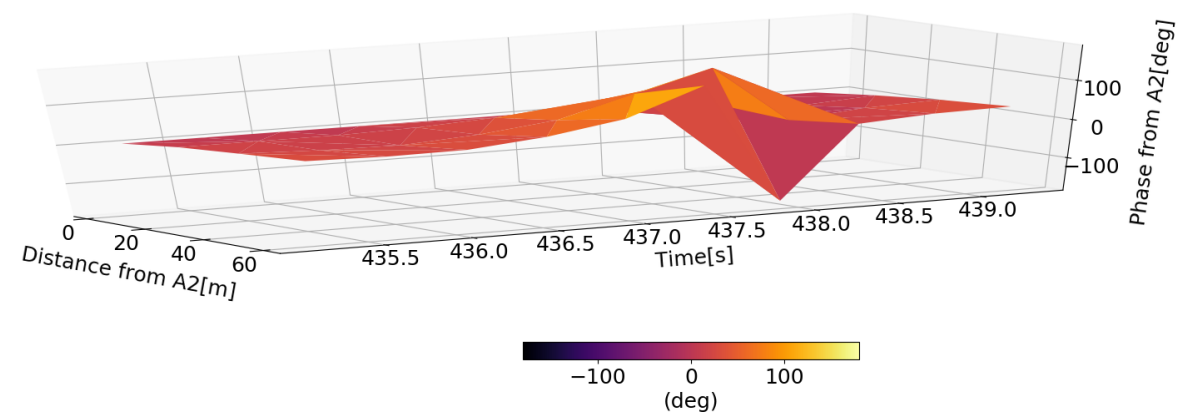

(d)

FIGURE 4.9: Animation frames of mesh from a 3.8 MHz signal showing a peak, consistent across the data from each antenna pair, travelling acaross the array. 


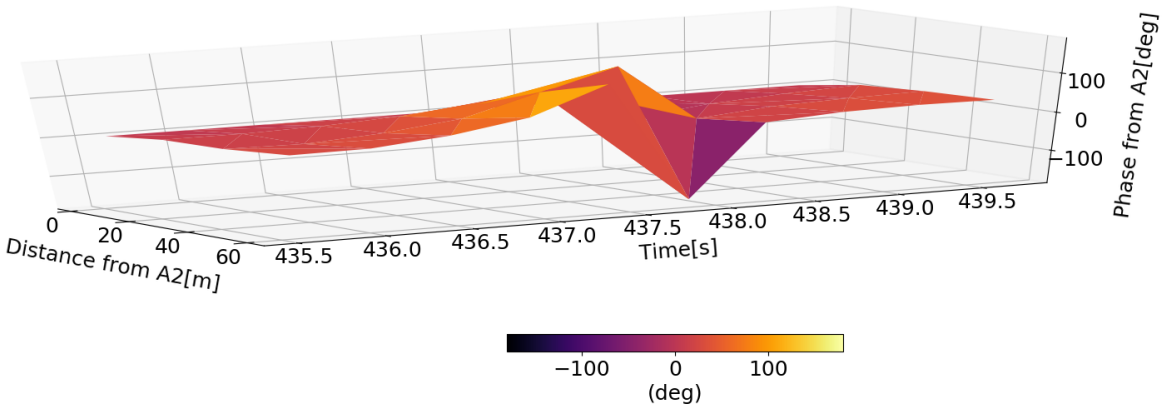

(e)

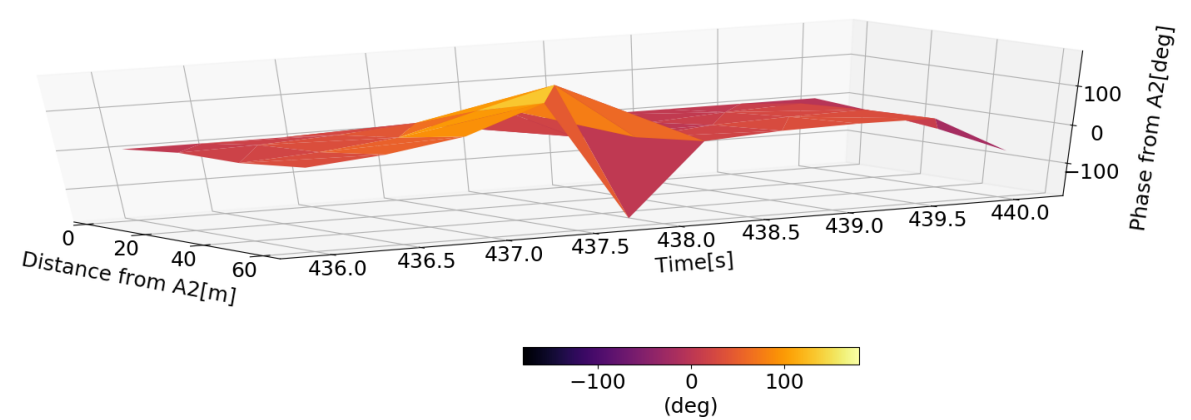

(f)

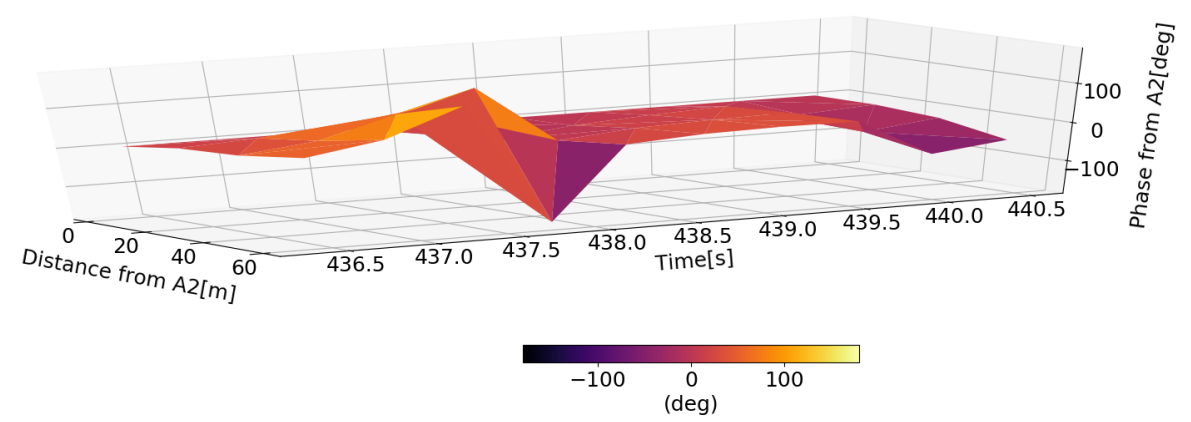

(g)

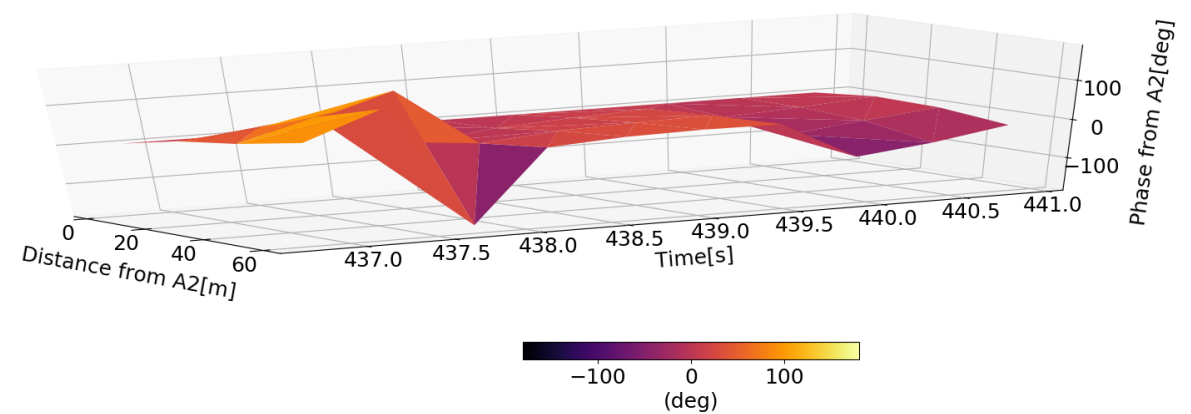

(h)

FIGURE 4.9: Animation frames of mesh from a 3.8 MHz signal showing a peak, consistent across the data from each antenna pair, travelling acaross the array (cont.). 

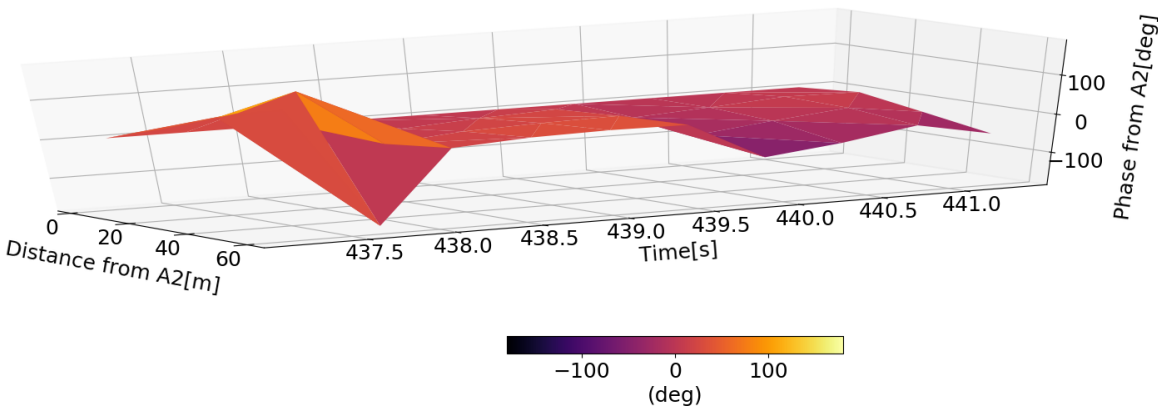

(i)

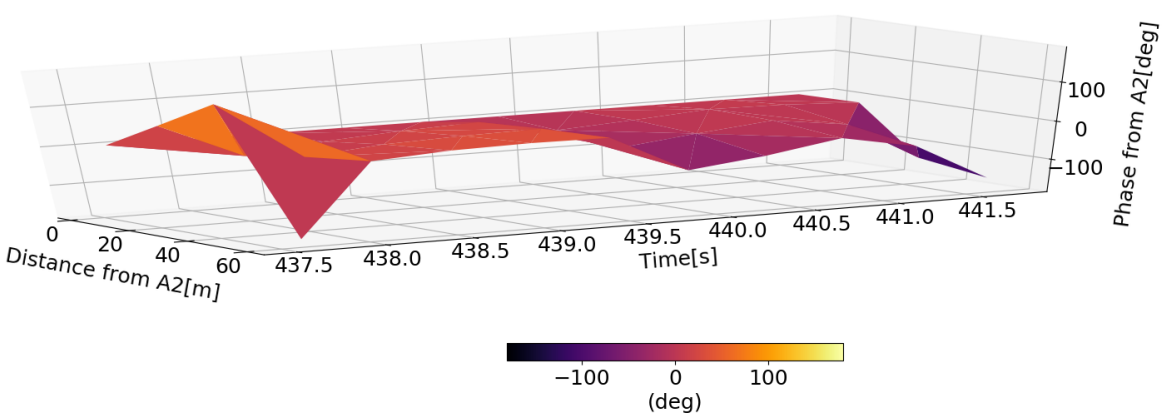

(j)

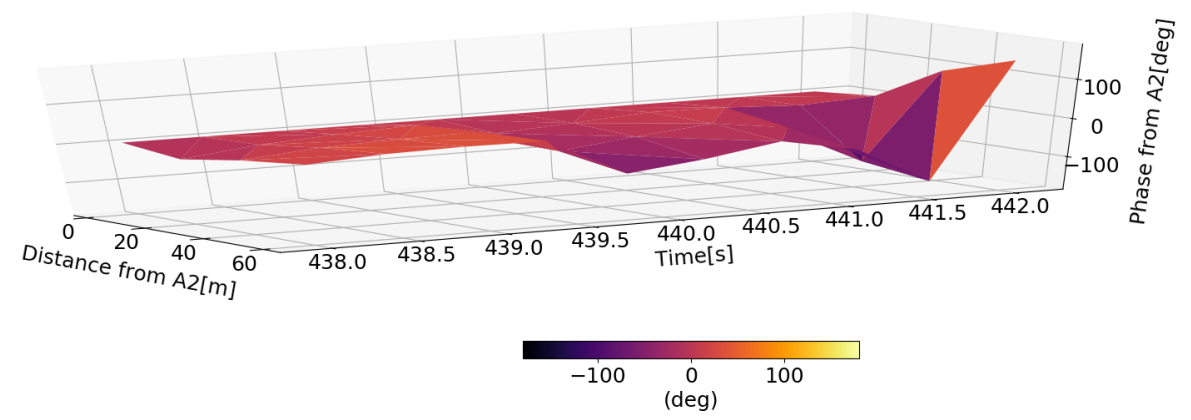

(k)

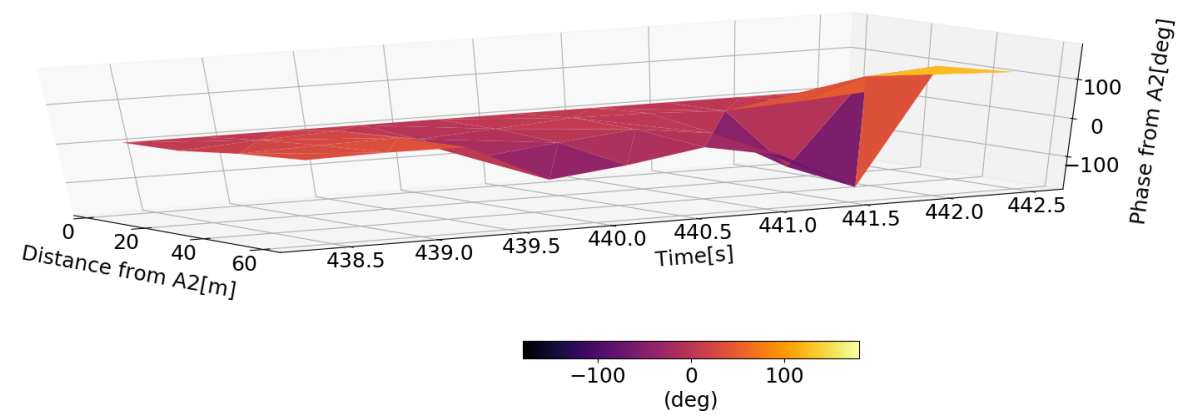

(l)

FIGURE 4.9: Animation frames of mesh from a 3.8 MHz signal showing a peak, consistent across the data from each antenna pair, travelling acaross the array (cont.). 

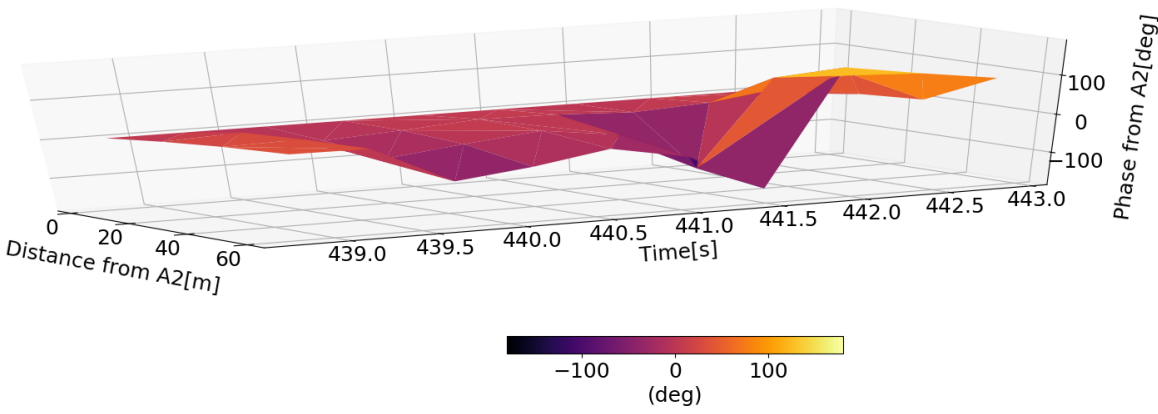

(m)

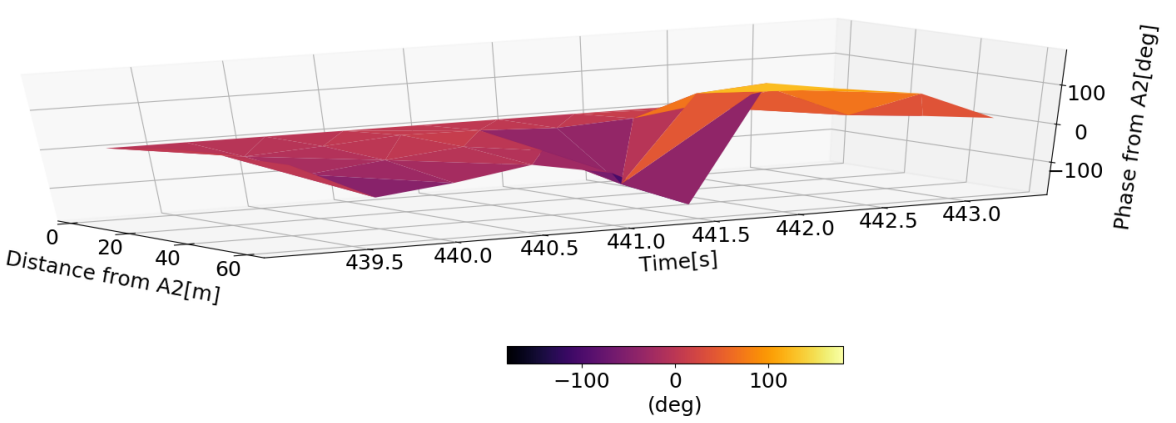

(n)

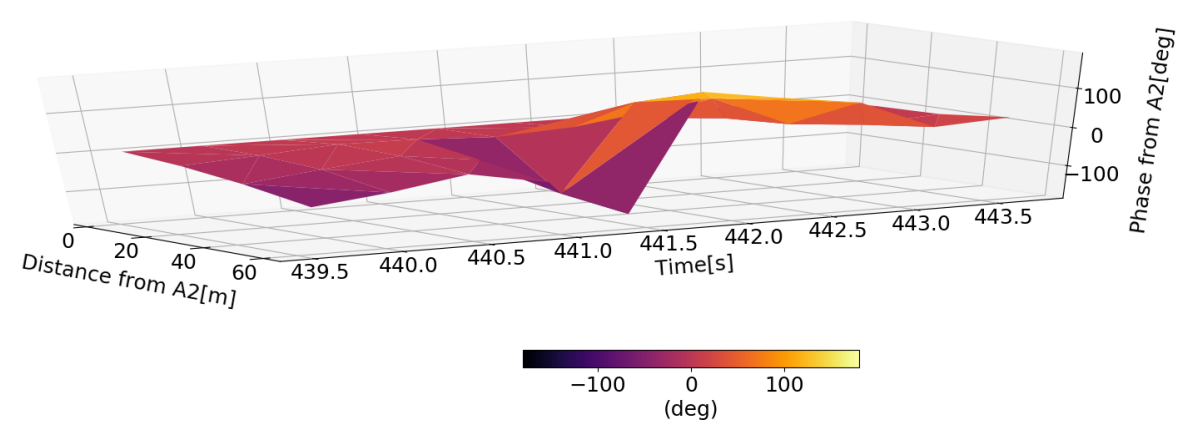

(o)

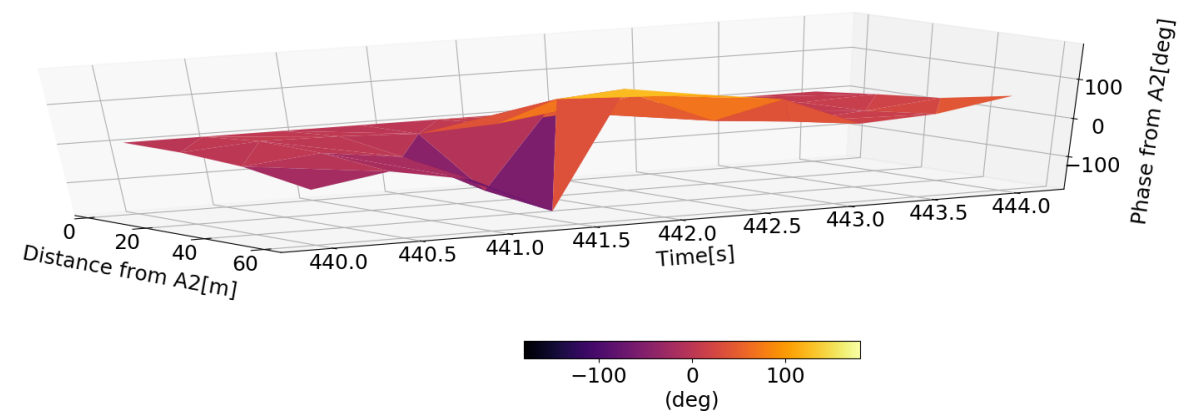

(p)

FIGURE 4.9: Animation frames of mesh from a 3.8 MHz signal showing a peak, consistent across the data from each antenna pair, travelling acaross the array (cont.). 

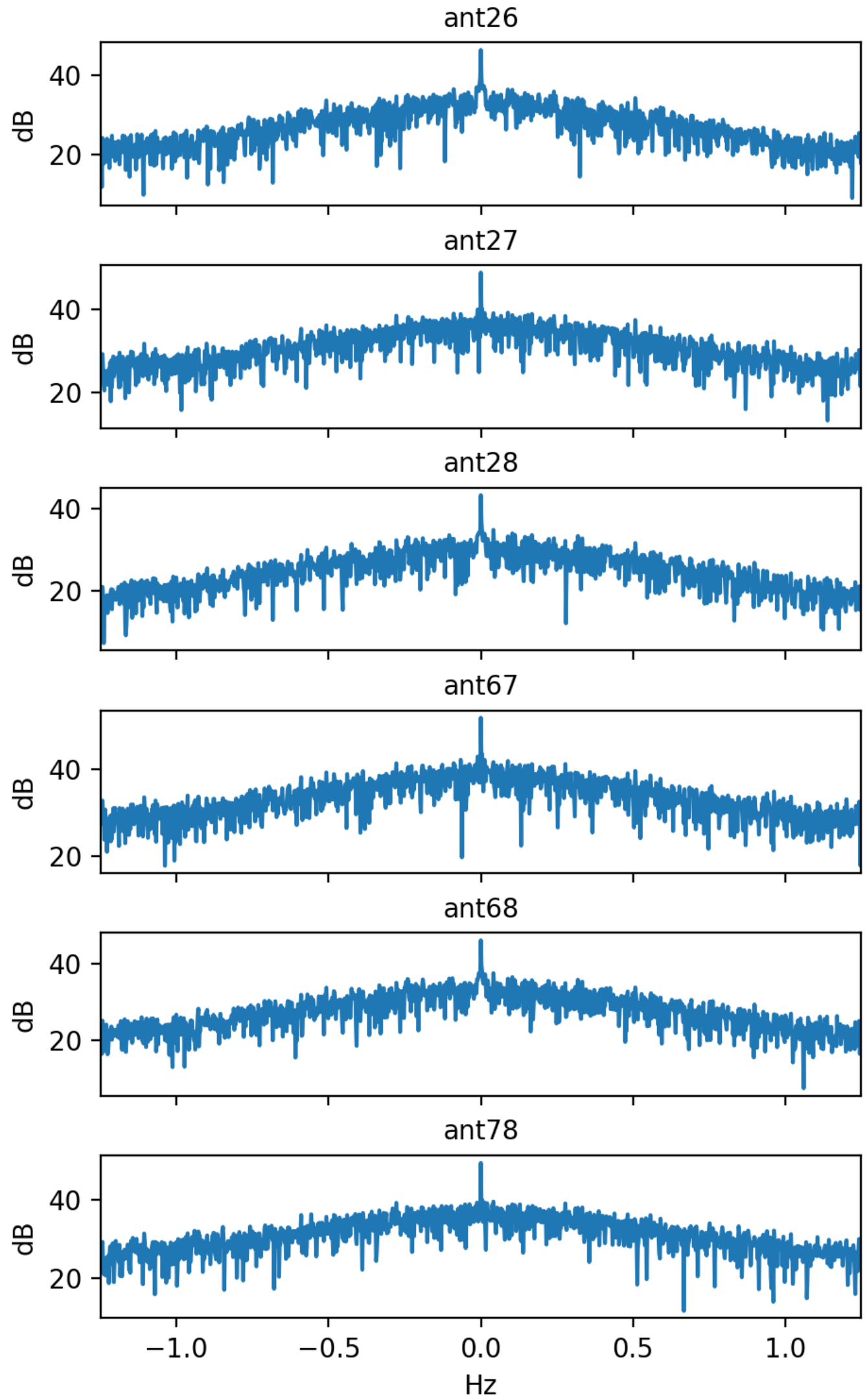

FIGURE 4.10: FFTs of all relative phase data showing no apparent frequencies of ionospheric waves under $1.25 \mathrm{~Hz}$. 


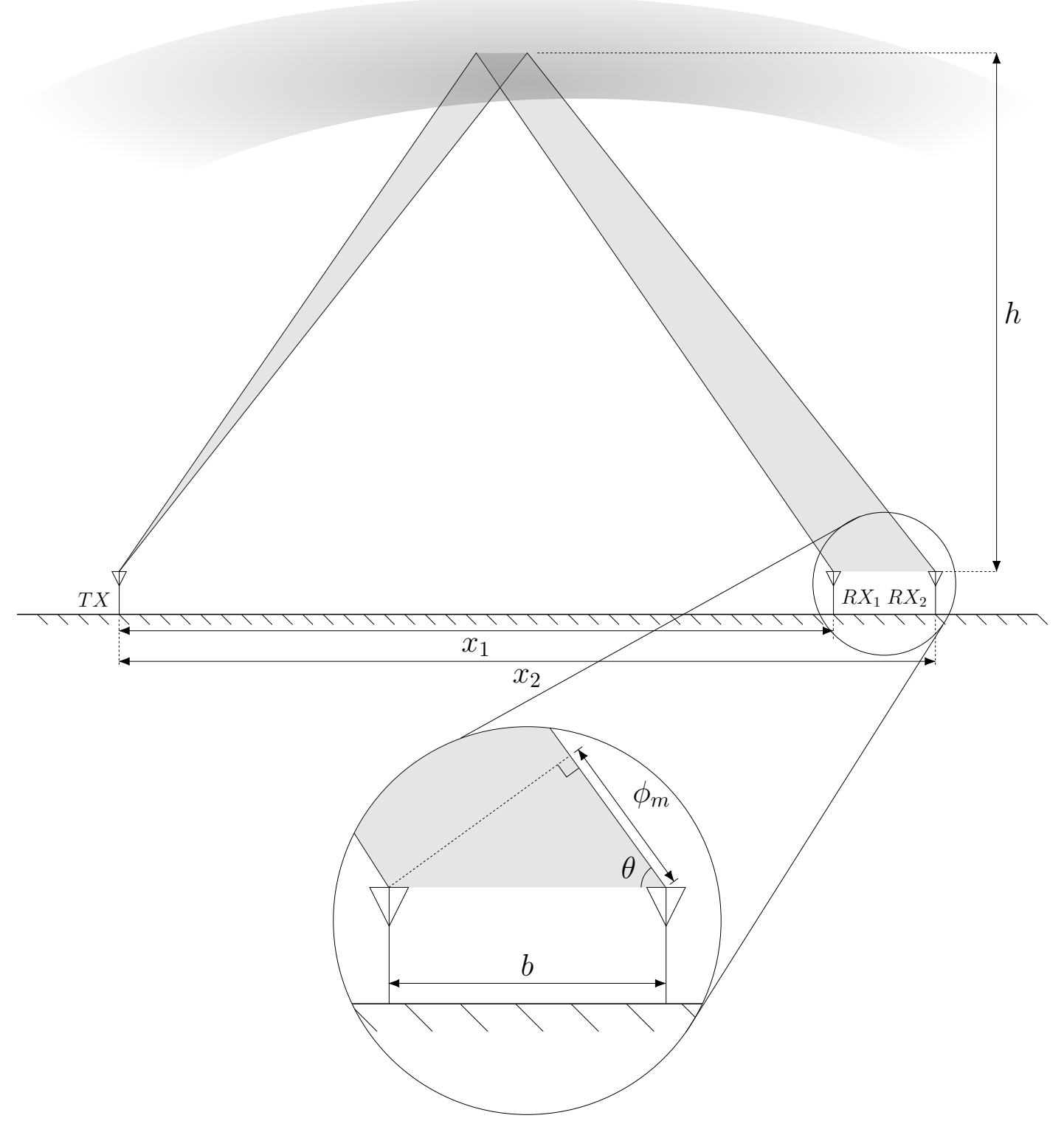

FIGURE 4.11: Ionospheric reflection height as function of physical geometry and RX-antenna phase difference. 


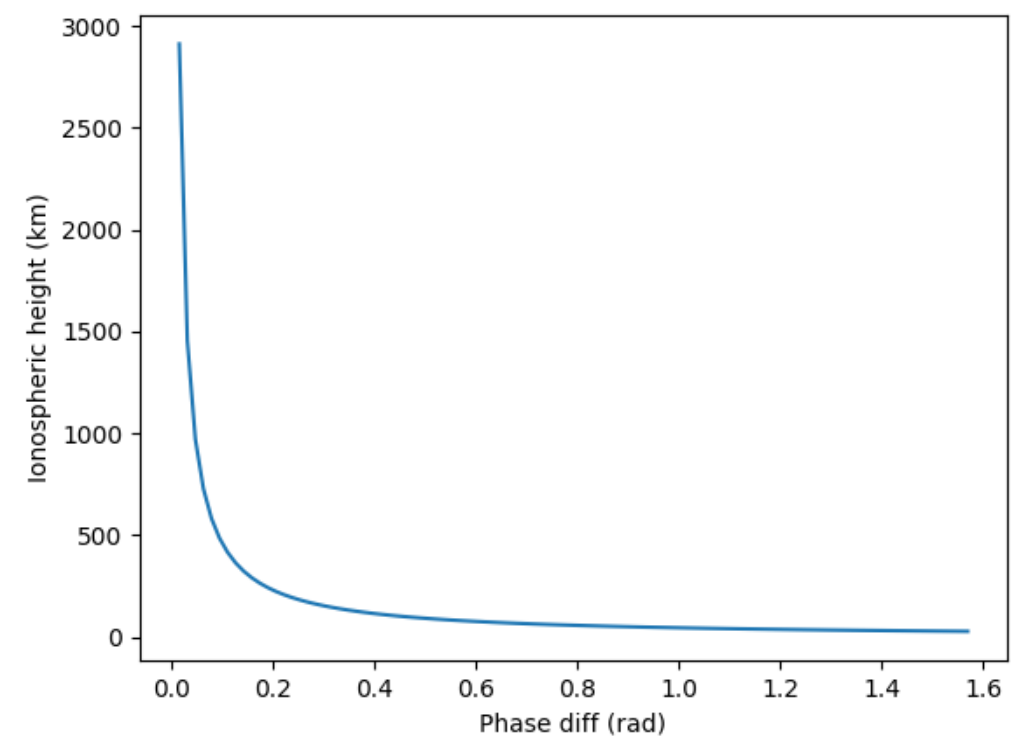

(a) Linear height vs. phase difference.

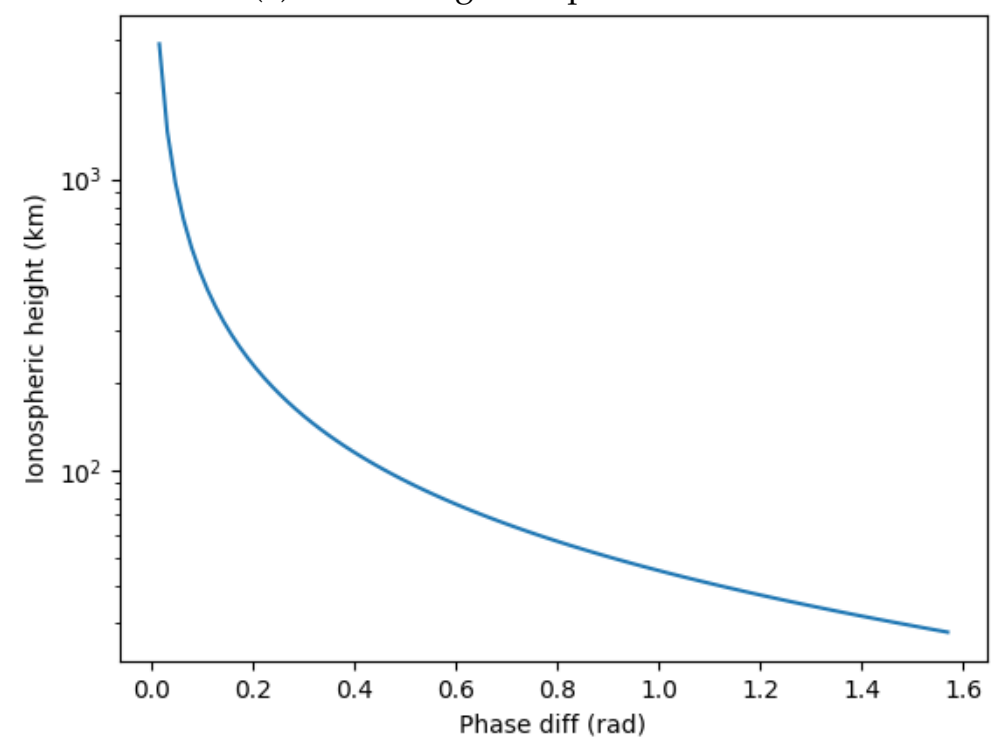

(b) Logrithmic height vs. phase difference.

FIGURE 4.12: Ionospheric height versus phase difference for a 3.7 $\mathrm{MHz}$ transmission received over a $60 \mathrm{~m}$ ground spacing, assuming a planar wave front. 


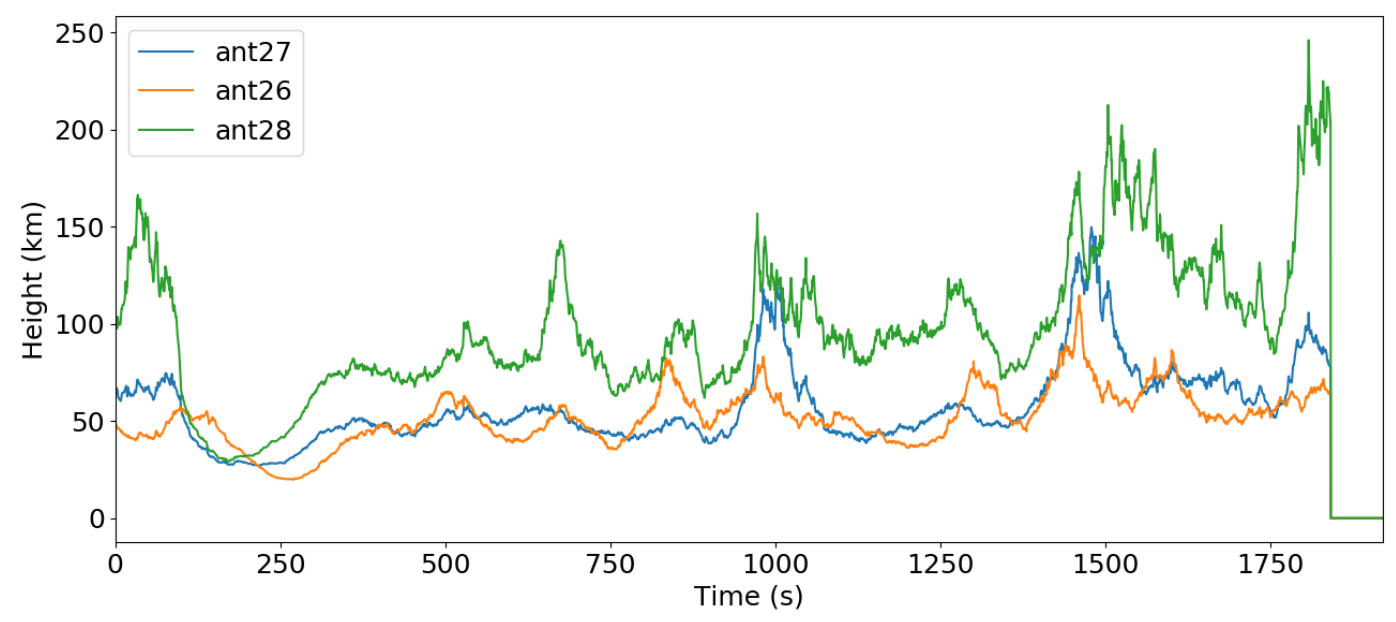

FIGURE 4.13: Ionospheric reflection height for a $3.7 \mathrm{MHz} \mathrm{CW}$ transmission assuming a planar wavefront.

between the two to continue iterating. This was abandoned when it was realized that using the largest value of $b(60 \mathrm{~m})$, and a $100 \mathrm{~km}$ ionospheric reflection height $(h)$, the difference in path length between a spherical and a planar wavefront is $0.00225 \mathrm{~m}$. This is found by taking a sphere of radius $200 \mathrm{~km}$ (total path length) and having it contact the closest antenna first. Then the distance between the sphere and the furthest antenna is the discrepancy between planar and a spherical wavefronts. The sensitivity of the instrumentation is not sufficient to see a difference at this scale. 


\section{Chapter 5}

\section{Conclusion and future work}

\subsection{Conclusion}

This thesis was driven by a desire to learn a new field of science (communications, electrical engineering, and geophysics) and to apply that learning to a project with large potential impact on society. A literature review resolved that the ionosphere is a medium with the potential to transmit messages about local weather, cosmic weather, and natural disasters. An experiment was built to attempt imaging the ionosphere using a phase-coherent array of HF antennas. The experiment was ultimately successful, using only four receiving antennas, ionospheric height was measured and the collected data demonstrated the phased-synchronicity of the array. No obvious ionospheric disturbances were measured, but there are several justifications for this. Foremost, the sampling rate of the collecting radio was low $(2.5 \mathrm{~Hz})$ which limits the size of ionospheric waves to extremely large or slow. It is also possible that no exciting events happened near enough to the receiving array during the relatively short experimental collection. Despite this, excitement about the project is high both at UVic and DRAO since the device was able to capture ionospheric height data in a novel way. A publication is being written collaboratively about this feasibility survey and the project is being scaled up to larger experiments. Special attention should be given to Appendix $C$ which lays out the recently accepted proposal for observation time on an existing large array in New Mexico. This will hopefully provide some high resolution (many pixels) data on a large phased array of antennas (256 elements).

The feasibility study outlined in this thesis was successful at demonstrating SDR's capabilities with respect to ionospheric measurements. It outlined the framework for a much broader study to be undertaken, and will have a large impact on society if successful, possibly allowing characterization of ionospheric disturbing events like earthquake precursors.

This feasibility study has given the ionospheric imaging project a strong start. The next step for the project is to begin collecting higher resolution data with the goal of characterizing the ionospheric perturbations. There are several conditions which must be met for this goal to become a reality. They are listed below.

\subsection{Future work}

- Make use of LWA observation time.

- Develop/buy an expandable multi-input SDR. Options include:

- CHIME ICE board (16 inputs)

- FlexRadio CDRX-3200 (32 inputs) 
- Nutaq PicoDigitizer 125-32-AC-SX315T (32 inputs)

- Nutaq PicoDigitizer 125-64-AC (64 inputs)

- Build a phased array in the HF range in a low-noise area.

- Consider synchronization techniques for the array.

- Run data collection runs for longer (O(hours), not $O$ (minutes)). 


\section{Appendix A}

\section{Setup and preparation for DRAO experiment}

Before conducting the experiment at DRAO (see Chapters 3, 4), a substantial amount of work was done to build the instrumentation and prepare. This appendix outlines the details of that effort.

\section{A.1 Experimental preparation}

In order to prepare for a the trip several things had to be agreed upon between DRAO and UViip. An important constraint imposed by DRAO was that the antenna installation could not break the ground in any way. Any hole in the ground would require a full environmental assessment and administrative approval process which was projected to take several months. Considering other time restraints, it was agreed that a free-standing system was preferable.

The receiver array was built around eight active antenna elements and everything was designed and built to ensure that any phase mismatches be attributable to ionospheric disturbance, not to a system imbalance. The final system cost was approximately $\$ 20,000 \mathrm{CAD}$.

The active antennas were bought from DX Engineering (model DXE-ARAH31P). They cover $0.1-30 \mathrm{MHz}$, which allows them to function in the 80 meter (3.5-4 $\mathrm{MHz}$ ) amateur radio band [28]. The antennas are broadband and high-impedance, allowing the $3 \mathrm{~m}$ active dipole to have an electrical length appropriate for any frequency in the 0.1-30 MHz range. A $12 \mathrm{~V}$ preamplifier employs a low noise FET input preamplifier with greater than $30 \mathrm{MHz}$ bandwidth to match the high impedance of the shorter $(3 \mathrm{~m})$ antenna to the low impedance input found on most radio receivers. The antennas were all built and then various mounting options were considered.

In order to be free-standing, several options were considered. One option was outlined which had antennas being tied to existing telephone-pole-sized masts already in the ground at DRAO. This option was later discounted as being too cumbersome since ladders would be needed for the installations and it would make moving the antennas difficult.

Tripods were the final choice for mounting platforms. The antenna specification sheet suggests that the antennas be mounted 20 feet above the ground for best performance which meant that the tripods were required to be stable up to that height.

The masts were bought from army surplus and are made of ribbed, hollow alluminum poles with collared ends. Fiberglass would have been preferable because of its complete lack of electro-magnetic property but they were not available at the time of purchasing. Each pole has a male end and a female end such that the poles can be fitted together. Table A.1 shows the dimensions of each pole. 
TABLE A.1: Army surplus tent-pole dimensions

\begin{tabular}{cc}
\hline Description & Inches \\
\hline Length & 48 \\
Male end length & 44.5 \\
Wall thickness & 0.009 \\
Outer diameter & 1.755 \\
Male end outer diameter & 1.56 \\
Female end inner diameter & 1.565 \\
\hline
\end{tabular}

The antenna specification sheet recommended a $20 \mathrm{ft}$ height which meant five poles were needed for each antenna, and another six poles were bought for each antenna in order to make a tripod base. Custom tripod base connectors were purchased to fit all of the legs together to support the mast (Figure A.1a). The tent poles also came with a fanning plastic base to add some extra support against tipping (Figure A.1b). A complete picture of the setup (without antennas) can be seen in Figure A.1c.

There was consideration for a cross-polarized system where each tripod would host two antennas mounted perpendicular to eachother. In order to do this while complying with the recommendation in the antenna specification sheet that the antennas each be mounted two feet above any conductive material, mast extensions were made out of wood. Wooden poles were cut to just over two feet long and a hole was drilled through both the wooden pole and the top of a tent-pole, which then hosted a cotter pin. This stopped rotation and held the mast-extension in place. The connection can be see in Figure A.2a while the cross-polarized antenna configuration can be seen in Figure A.2b. To aid with cable management and have a centralized power distribution system, a mounting platform was built for all of the power inserters (shown later in Figure A.3d).

The coax bought was $75 \Omega$ flooded RG-6/U coax. The flooding makes the coax self-healing from small cuts to the jacket but sticky to work with. The coax was shipped in $1000 \mathrm{ft}$ rolls and it was decided that the cable lengths should be approximately $325 \mathrm{ft}(100 \mathrm{~m})$. To cut them to length a rope measuring device was used. In total, 9 lengths were cut and teminated using F-type connectors. These lengths were for patching the antennas to the bias power boxes which had to be inline to insert $12 \mathrm{~V}$ and increase the effective antenna length to be appropriate in the 3.5-4 MHz range.

Some of the excess material was used to patch the distance between the power inserter and the SDR. The power inserter has an F-type connector going to the antenna and an RCA connector going to the radio. $10 \mathrm{ft}$ patch cables were made with an RCA terminator and a BNC terminator.

After physically building each component of the system, all eight antennas were setup on the roof of UVic's Engineering Laboratory Wing (ELW) for some local tests (Figure A.3). The masts were reduced by eight feet (two pole segments) to make adjusting the antennas easier during preliminary tests. It was understood that this would enter some element of noise to the system, but the environment was relatively noisy already and so the test was more done to check that all of the antennas worked.

The antennas were all tested on the roof of the engineering wing at UVic to check 


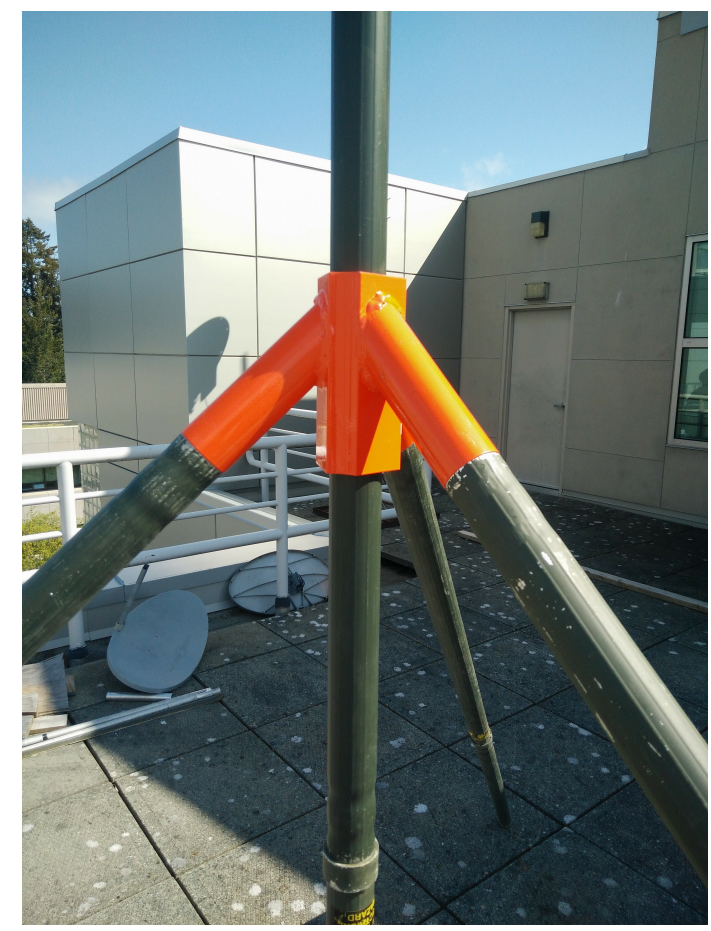

(a) Custom tripod base connector.

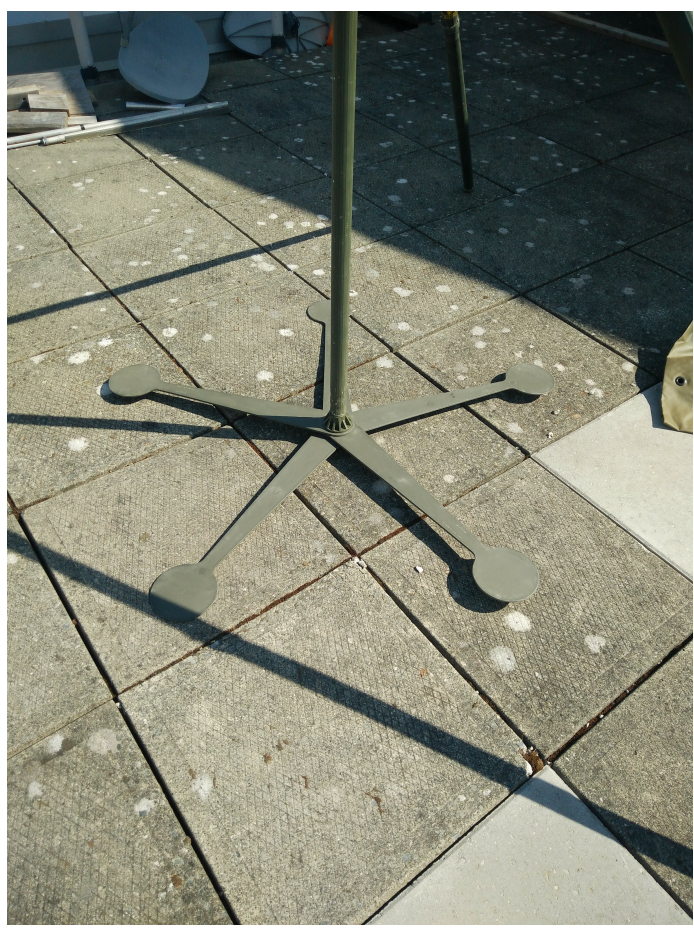

(b) Custom fanning mast base.

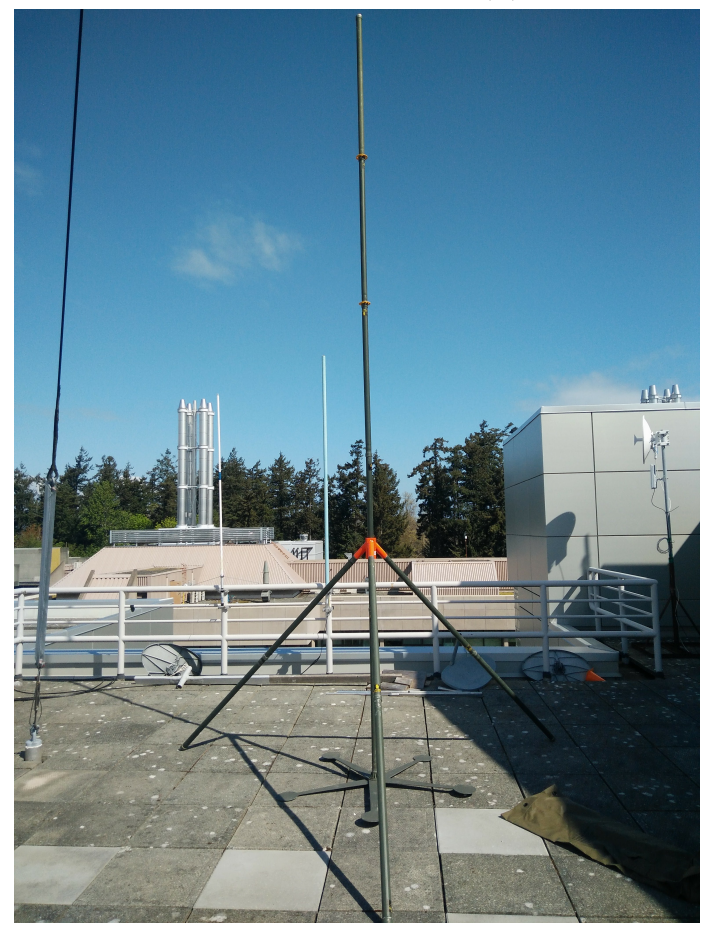

(c) Configured tripod made of surplus army tent poles.

FIgURE A.1: Mast support 


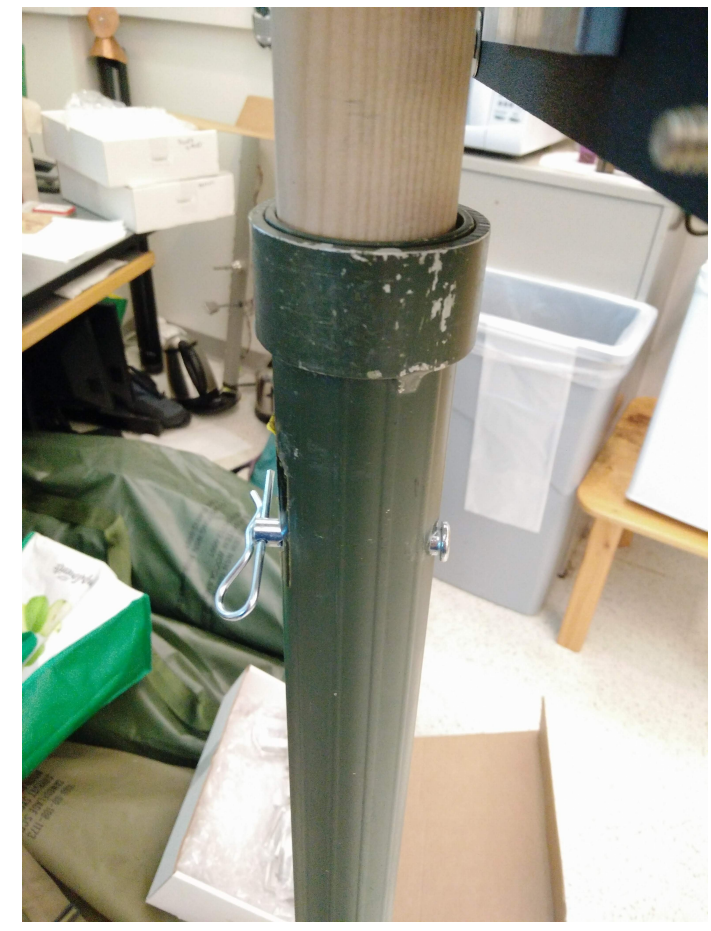

(a) Cotter pin holding a mast extension in place.

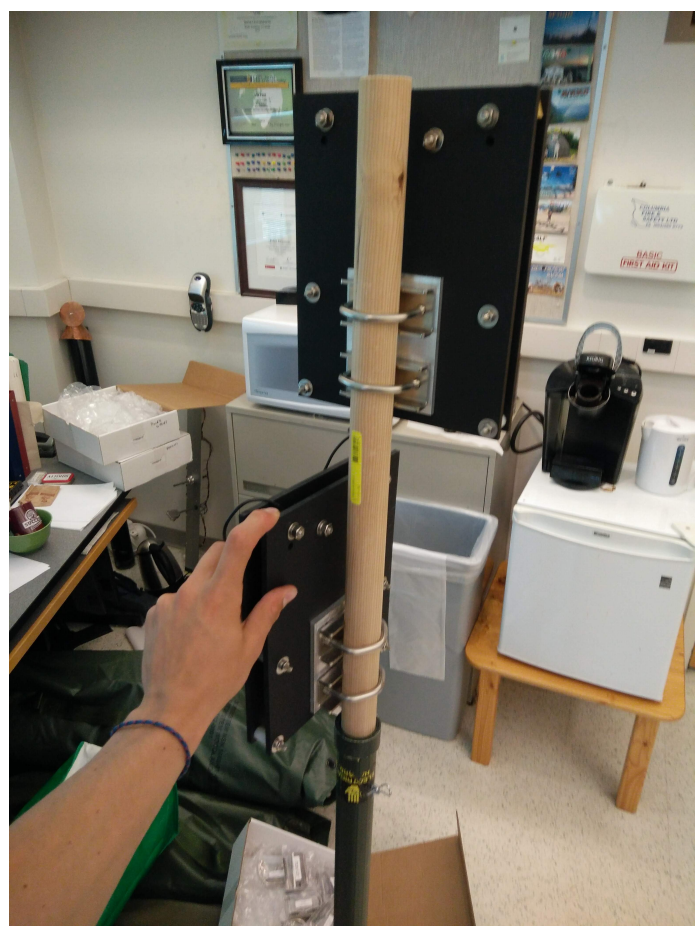

(b) Two antennas mounted cross-polarized on a mast extension.

FIGURE A.2: Mast extension

that the noise-levels were similar. The tests were run at dusk (8:40-9:05 pm) by plugging each antenna (one-by-one) into the UVic KiwiSDR (kiwisdr.ece.uvic.ca) input and checking the noise level and strongest apparent signal between 3.5 and 4 MHz. These values are given in Table A.2, and a sample image is shown in Figure A.4. The strongest signal was found by waiting for a peak to appear and quickly recording it.

The next step in experimental preparation was to plan the actual layout of the experiment when everything had been transported to DRAO. To do so, several assumptions were made:

- the waves on the ionosphere were constant in time,

TABLE A.2: Values from the antenna tests.

\begin{tabular}{ccc}
\hline Antenna & Noise level $[\mathrm{dBm}]$ & Strongest signal $[\mathrm{dBm}]$ \\
\hline None & -120 & -113 \\
1 & -103 & -76 \\
2 & -108 & -81 \\
3 & -108 & -73 \\
4 & -103 & -75 \\
5 & -103 & -79 \\
6 & -103 & -86 \\
7 & -107 & -81 \\
8 & -104 & -73 \\
\hline
\end{tabular}




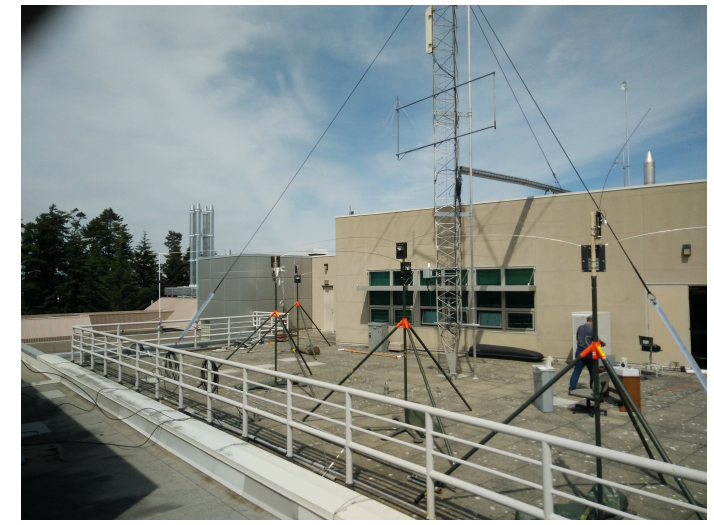

(a) Cross-polarized antennas.

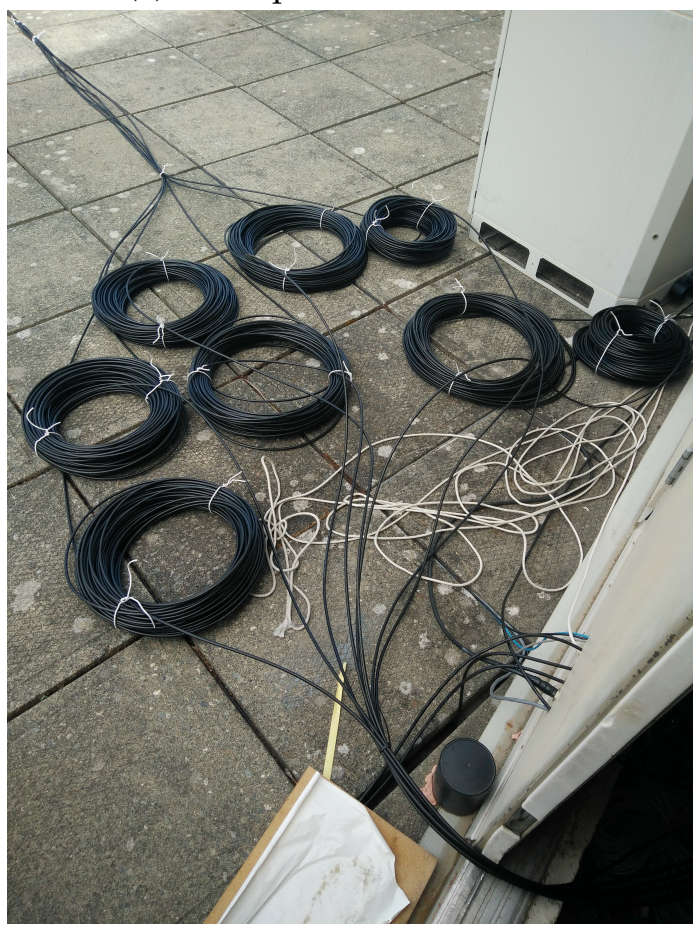

(c) A roll of coax from each antenna.

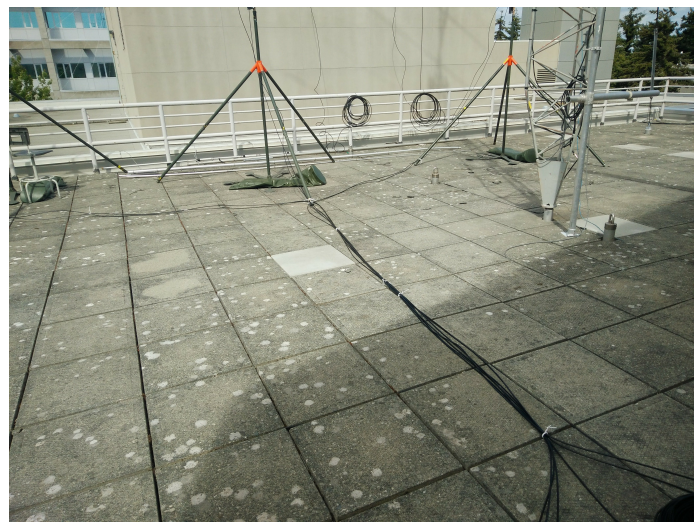

(b) Cable management.

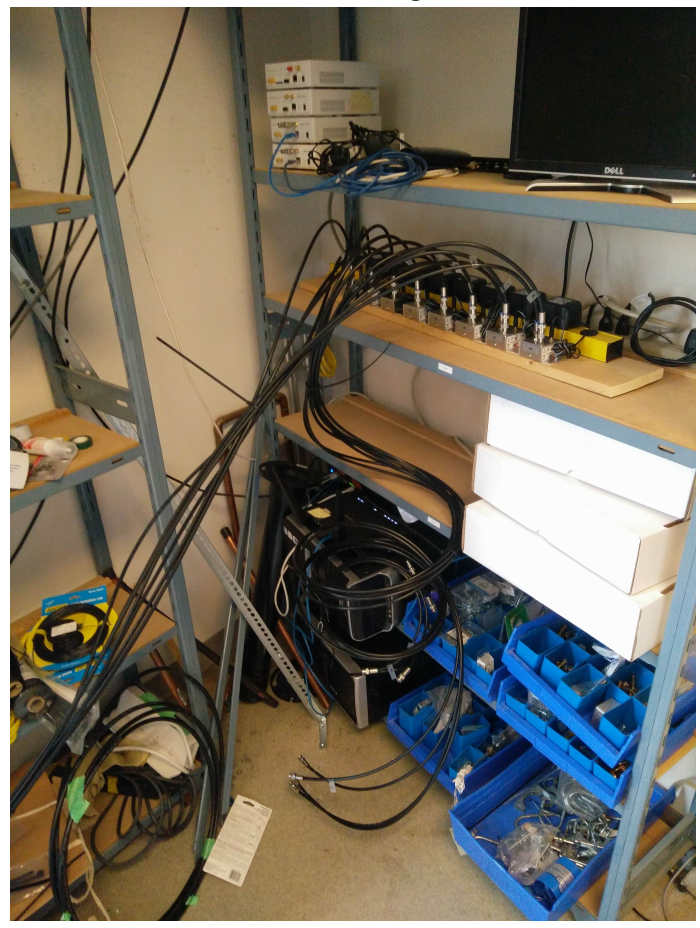

(d) Power management system.

FIGURE A.3: First system setup on roof of ELW. 

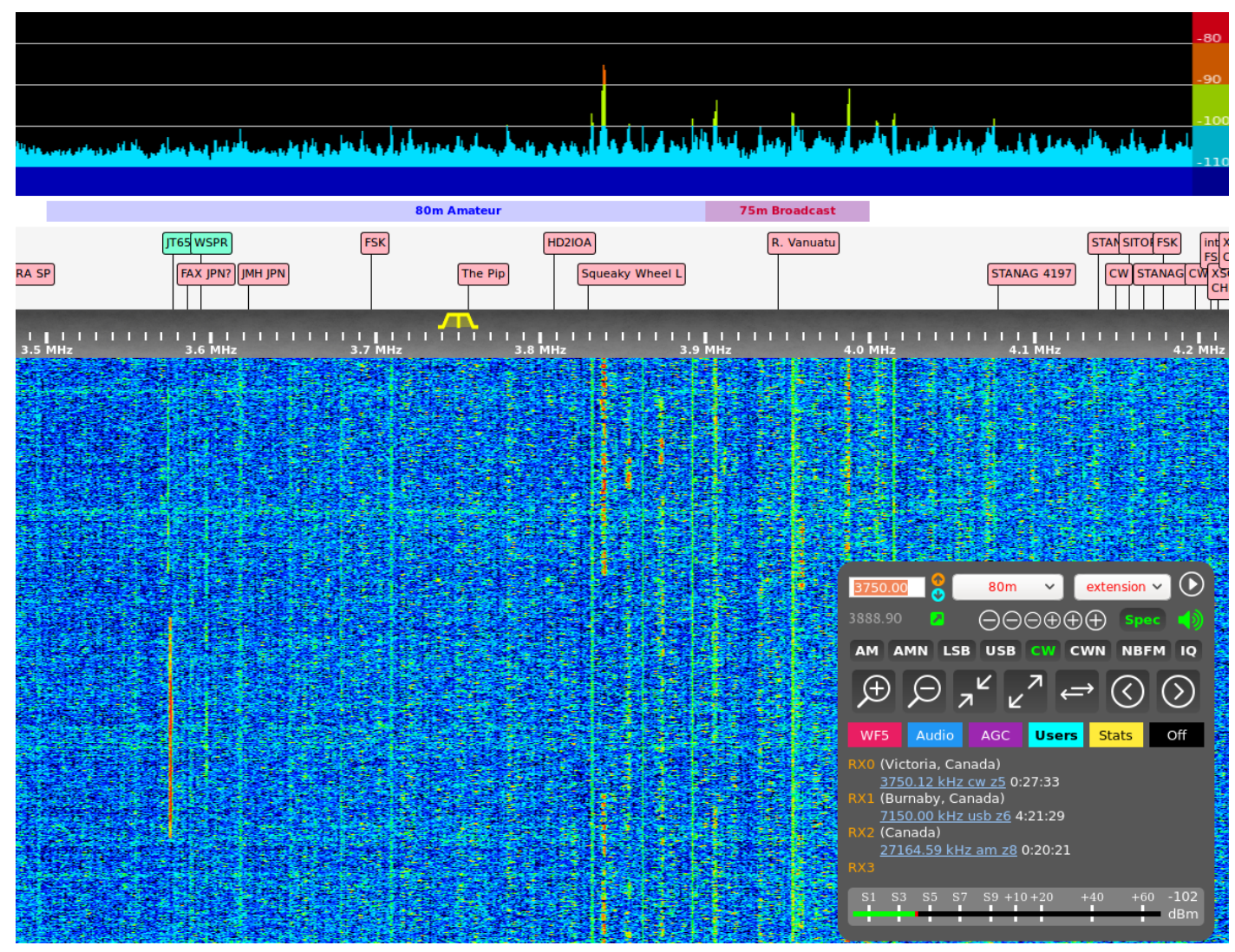

FIgURE A.4: Antenna 4 on the UVic KiwiSDR. A strong signal (-85 $\mathrm{dBm}$ ) is apparent around $3.84 \mathrm{MHz}$.

- the waves are propogating linearly over the experimental site (a constant direction),

- moving the antennas would be simple and relatively fast,

- the field hosting the experiment would be easy to move around in,

- it would be easy to move antennas outside of the field in most directions.

Operating under these assumptions, the first map of the area was provided by Dr. Stephen Harrison via Google Maps (Figure A.5).

With the assumption that the ionospheric waves were constant in time, it was reasonable to assume that if the experiment was started, stopped, and started again, the same waves would be imaged. From this it was decided that the best way to capture good images of ionospheric waves using the limited number (eight) of antennas available would be to use the following procedure:

1. setup all of the antennas in a line,

2. capture data,

3. rotate the array by a certain number of degrees,

4. capture data,

5. repeat steps (1) to (4) until the ionospheric waves are captured when travelling parallel to the array. 


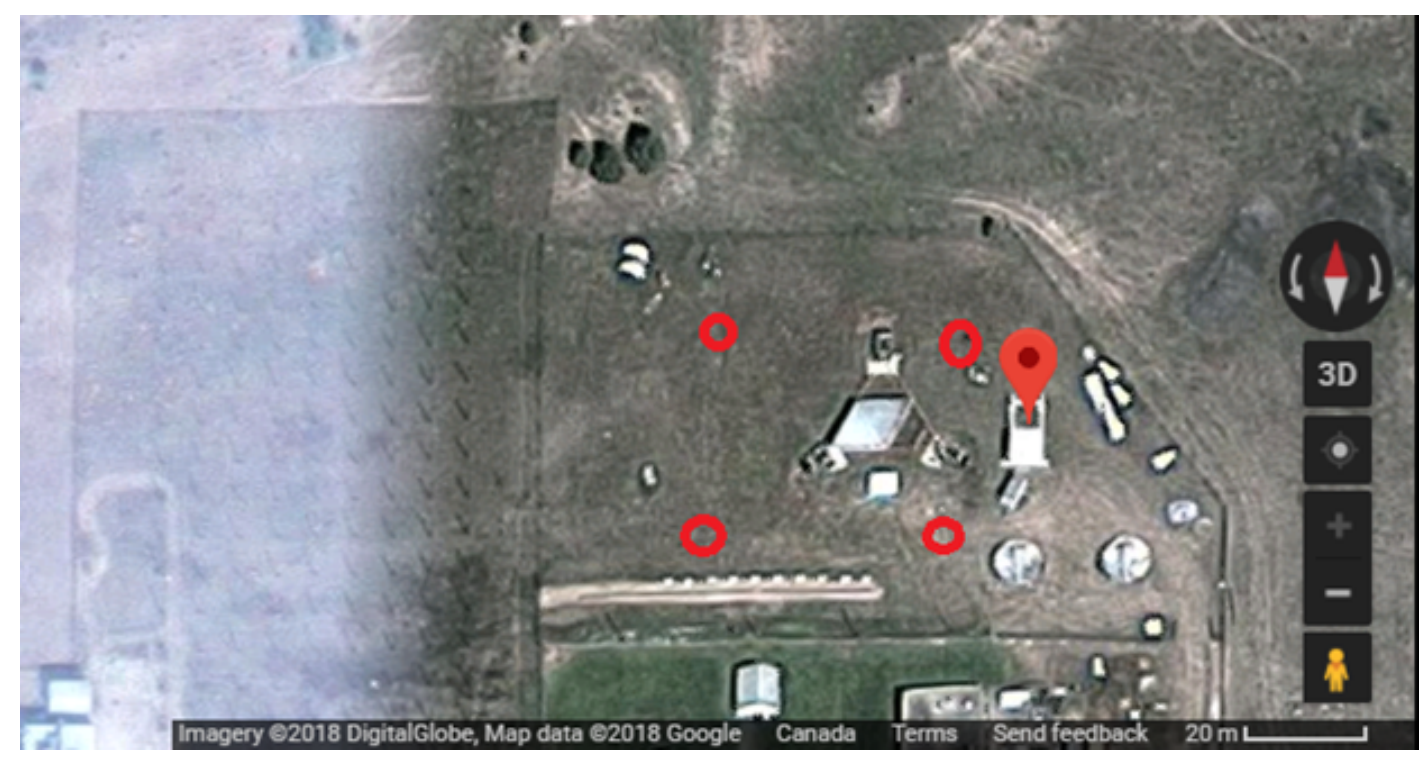

FIGURE A.5: Map of the experimental area. Red circles indicate antenna locations proposed by Dr. Stephen Harrison, the red marker indicates the shed which would provide access to power.

TABLE A.3: Wavelengths relevant to the experiment $\left(c \approx 3 \times 10^{8}\right)$.

\begin{tabular}{ccc}
\hline$f[\mathrm{~Hz}]$ & $\lambda[\mathrm{m}]$ & $1 / 4 \lambda[\mathrm{m}]$ \\
\hline $3.5 \times 10^{6}$ & 85.7 & 18.75 \\
$4 \times 10^{6}$ & 75 & 21.4 \\
\hline
\end{tabular}

Rotating in this manner would mean that the ionospheric wave would be caught at some angle, but eventually the array would be rotated until either parallel to the array or close to parallel. Once parallel, the array would be capturing time data of the ionospheric wave and seeing as close to true wave amplitude/shape as possible.

Relevant to the array setup at DRAO was knowing the physical spacing between antennas. A quarter wave-length spacing allows for above Nyquist imaging. Knowing that wavelength is a function of frequency $(\lambda=c / f$, where $\lambda$ is the wavelength, $c$ is the speed of light in the relevant medium, and $f$ is the frequency of the wave), the quarter wavelengths were found (table A.3). Being restricted to the amateur radio band of 3.5 to $4 \mathrm{MHz}$ made the difference in possible wavelengths small. It was decided that to increase resolution, the antennas would be arranged closer to $15 \mathrm{~m}$ apart.

To make the experiment run smoothly once at DRAO, maps were prepared to plan the specific antenna placements. Assuming a constant ionospheric wave, the antenna array would have to be rotated just under $180^{\circ}$. This would capture the waves both parallel and perpendicular to the array. Figure A.6 shows the proposed layouts plan view. At this point the experiment was ready to be conducted and the equipment was transported to DRAO. 

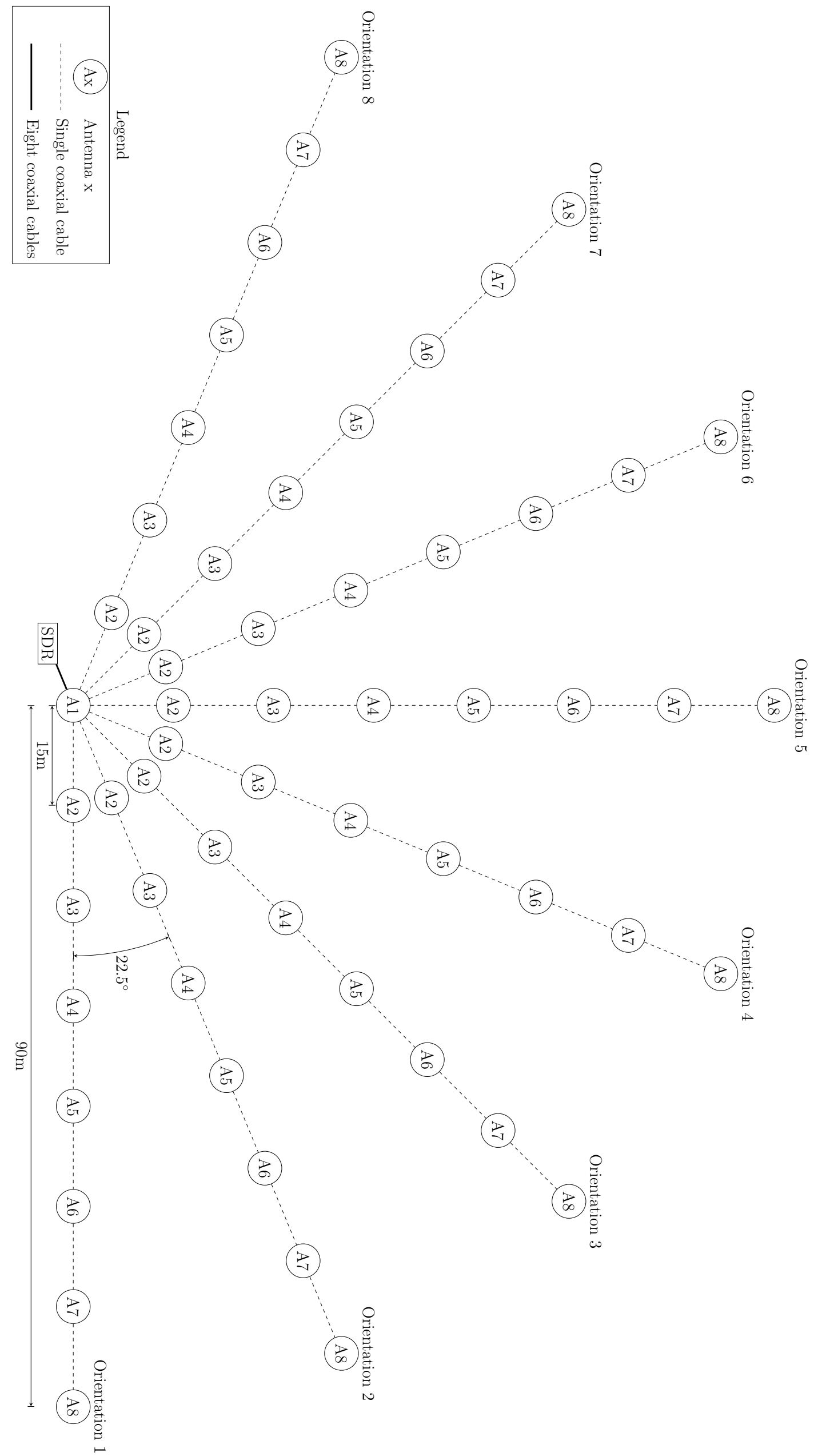

FIGURE A.6: Plan view of eight planned antenna orientations. 


\section{Appendix B}

\section{Preliminary study at UVic}

Before buildling and running the feasibility study outlined in this thesis, a smaller study was done at UVic. All of the work in this appendix was done collaboratively with Peter Kremler. A transmission site was setup approximately $10 \mathrm{~km}$ from UVic and two receiving antennas were setup on a rooftop at UVic. A report was written on the effort, which has been shortened and revised for this appendix.

\section{B.1 Equipment}

This project was conducted between the roof of the Engineering Lab Wing at the University of Victoria, and a private house in the Oaklands neighbourhood of Victoria. In order to execute the experiment, transmitting and receiving antennas had to be set up, along with the hardware and software to use them.

A primary concern was operation in a noisy environment (Victoria, B.C.). In order to assist with this, pulse compression was used to operate at a relatively low power. By transmitting over a longer period of time, pulse compression allows the signal to noise ratio of low power signals to be increased upon reception, if they are highly autocorrelated. Therefore it was decided to use a chirp (linear frequency modulated signal, equation B.1) from 3.5-4 MHz given by

$$
\Phi(t)=e^{j \pi\left(\frac{f_{2}-f_{1}}{T}\right) t^{2}}
$$

\section{B.1.1 SDR system}

For this project, the main hardware used was the Ettus USRP N200/N210 Software Defined Radio system [29]. This device provides the high-speed A/D and D/A conversion as well as the analog front end necessary to translate between the complex baseband signals and the real passband signals that are required for transmission.

The output of the USRP, measured across a $50 \Omega$ dummy load is approximately $1.15 \mathrm{Vpp}$ which is equivalent to $5 \mathrm{dBm}$.

\section{B.1.2 Power amplifier}

With an output power of approximately $5 \mathrm{dBm}$, the N200 required amplification to achieve the required transmission power. To amplify the signal, the 2100L RF Power Amplifier [25] was used, providing $50 \mathrm{~dB}$ of gain for frequencies between $10 \mathrm{kHz}$ and $12 \mathrm{MHz}$. This amplifier is rated for $100 \mathrm{~W}$, providing enough power to generate a strong signal. The high gain also meant that the USRP did not have to transmit at maximum power, thereby avoiding clipping. 


\section{B.1.3 Antennas}

In selecting antennas for this project, the following requirements and constraints were considered:

- frequency range of $3.5-4 \mathrm{MHz}$,

- transmit power and receiver sensitivity to perceive radio waves returning from the ionosphere,

- space and portability constraints.

For the initial round of testing, it was decided to use a single transmit antenna and two receive antennas, allowing comparison between the receivers.

\section{Transmission}

Initially a linear dipole antenna was used, configured broadside to the university. While this provided some preliminary results, space constraints at the transmission site meant that the antenna could not be properly tuned to the desired frequency range. For this reason, it was decided to use a loop antenna stretching around the perimeter of the property, which provided enough length to allow proper tuning.

\section{Reception}

The signal was received from the roof of the Engineering Lab Wing at the University of Victoria, outside of the radio propagation lab. The antennas chosen were from DX Engineering [30], and use a low noise preamplifier to boost the weak signals provided by an antenna whose dimensions are a fraction of the wavelength of the signals being studied. The tradeoff for this was greater portability. This was especially important as the best spacing of the two antennas was initially unknown and was one of the experimental parameters that needed to be tweaked to provide the best results.

\section{B.2 Experimental results}

This project was completed in increasingly complex stages to allow for verification. Each stage followed the same algorithm of generating a high autocorrelation, complex baseband signal, transmitting it over a medium, receiving the signal, then performing pulse compression by correlating the received and transmitted data, and analyzing the results to determine information about the channel. Because the transmitted signal was complex, the cross-correlation provided information both about the magnitude and phase of the transmitted signal. The four stages, simulation, direct-line, short range line-of-sight, and long range multipath transmission are detailed in the following sections.

\section{B.2.1 Simulation}

Two different sumulation techniques were employed to validate the experiment. The first was done to practice the correlation method for a known signal while the second was done to simulate ionospheric refraction of a radio wave. 


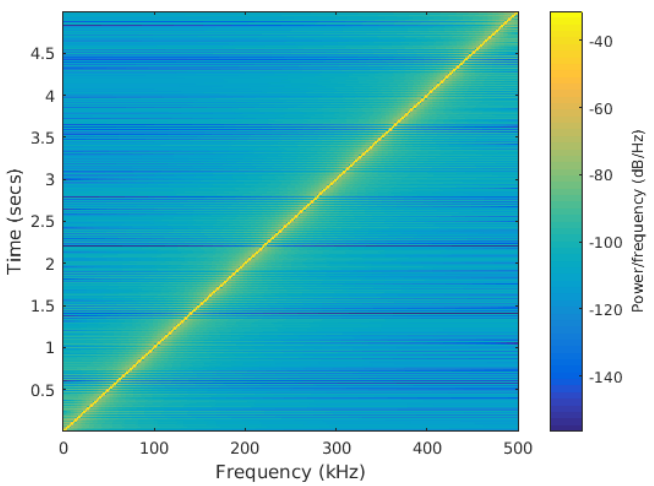

(a) Transmitted signal.

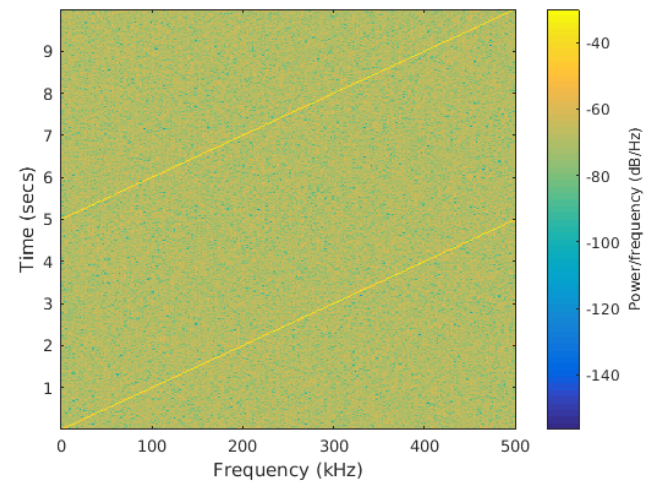

(b) Received signal.

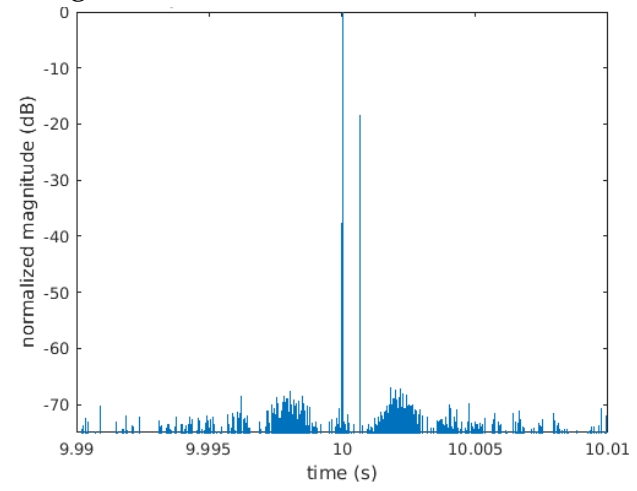

(c) Cross-correlation.

FIGURE B.1: Multipath channel model results.

\section{Multipath channel model}

The first simulation was to run the experiment in Matlab with the multipath channel modelled as a tapped delay line. The purpose of the simulation was both to verify the effectiveness of the correlation technique, and also to develop some intuition for the appearance of the output data. Furthermore, although this was the first stage attempted, the simulation was revisited and updated throughout the project as a tool to understand the data output.

For algorithm verification, a very simple, multi-channel, two-ray model was used. For this model, the input signal was copied to the output file, and added to an attenuated, time-delayed copy of itself, mimicking the behaviour of a direct path wave added to a reflected path wave. Finally the output signal was obscured by additive Gaussian white noise. Using this simple model, the correlation technique was verified for a known signal.

\section{Watterson ionospheric model}

In order to verify that the data interpretation is being done correctly, an ionospheric model was also created. The simulation was used to show that each peak in the correlation, or impulse response, corresponds to a distinct atmospheric layer or region and that the time between the peaks corresponds appropriately with the distances between the layers or regions.

The simulation model chosen was the Watterson model, published in 1970 [31]. It is a high frequency model in which the transmitted signal is fed into a tapped delay line. Each tapped delay represents an ionospheric region and consists of a Gaussian 
TABLE B.1: Watterson model simulation inputs.

\begin{tabular}{cccc}
\hline Ionospheric Region & Altitude $(\mathrm{km})$ & Thickness $(\mathrm{km})$ & Electron density $(\%)$ \\
\hline$D$ & 90 & 0.1 & 1 \\
$E$ & 120 & 20 & 5 \\
$F_{1}$ & 220 & 20 & 40 \\
$F_{2}$ & 350 & 30 & 54 \\
\hline
\end{tabular}

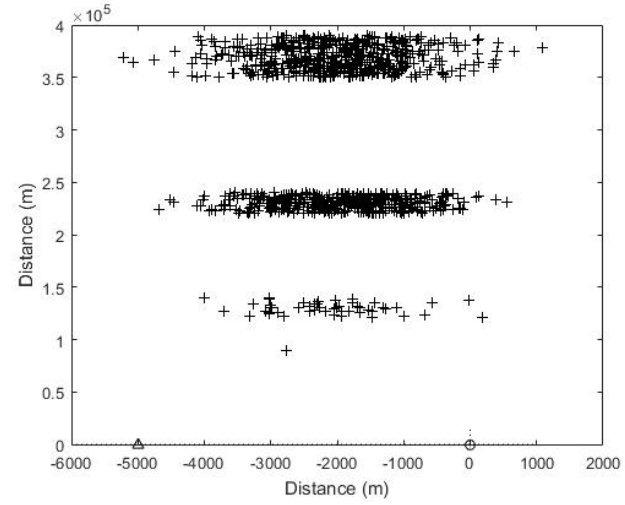

(a) Scattering scenario showing ionospheric heights.

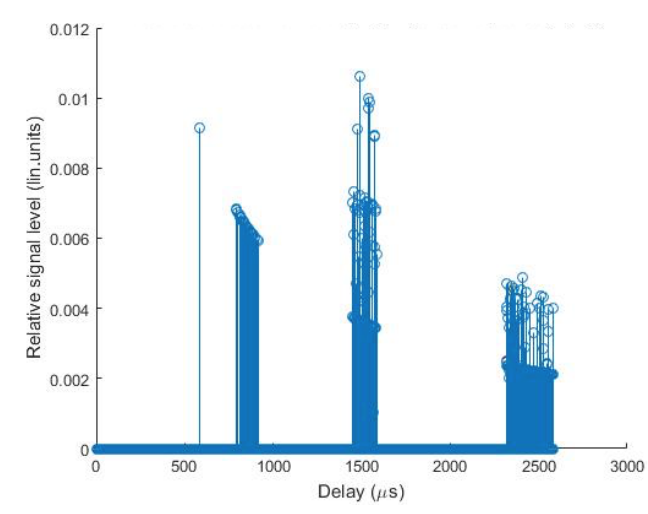

(b) Absolute value of impulse response.

FIGURE B.2: Watterson model simulation.

function. Each function has components of Rayleigh fading and Doppler spreads, to simulate the radio wave scattering at each refraction point.

To simplify the simulation an assumption was made that radio wave refraction occurs only in designated regions, and radio waves travel freely between regions. For this particular simulation the refraction heights, thickness of the refraction region, and electron densities were predefined. Table B.1 shows the values used and the results are given in Figure B.2a. It shows the ionospheric region densities graphically. The impulse response was plotted as well (Figure B.2b), which simulates the way the experimental data may look post-correlation.

\section{B.2.2 Expected values}

The 2-ray multichannel signal was transmitted over a single coax cable to simulate the effect of the ground path and reflected path. The calculations below, in which $D_{P x}$ and $R_{P x}$ are the groundwave and skywave path distances respectively, show the approximate time delay between the ground and skywave

$$
\begin{aligned}
D_{P 1} \approx D_{P 2} & =3.05 \mathrm{~km} \\
R_{P 1} \approx R_{P 2} & =200 \mathrm{~km} \\
v & =3 \times 10^{8} \mathrm{~m} / \mathrm{s}
\end{aligned}
$$




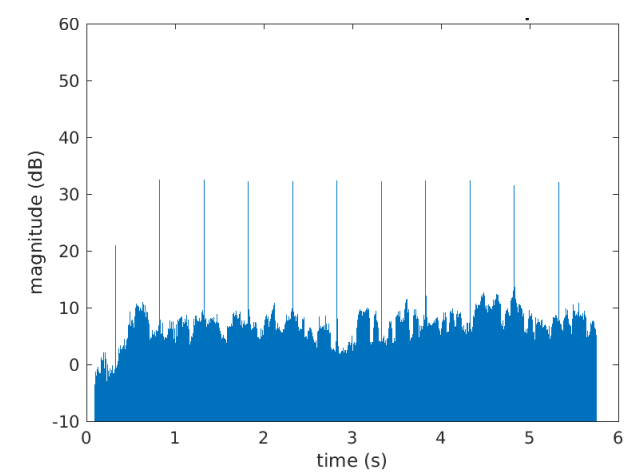

(a) Correlation between transmitted and received signals.

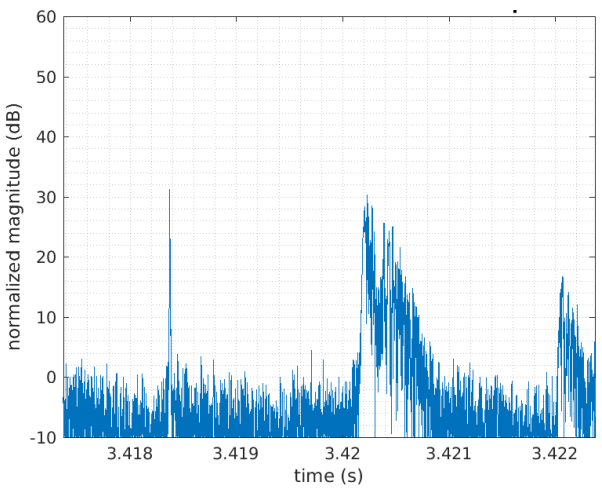

(b) Correlation between the transmitted and received signals where a single peak has been zoomed in on.

FIGURE B.3: Experiment length correlation and single peak detail.

$$
\begin{gathered}
t_{D}=\frac{D_{P x}}{v}=1.017 \times 10^{-5} \mathrm{~s} \\
t_{R}=\frac{R_{P x}}{v}=6.67 \times 10^{-4} \mathrm{~s} \\
t_{\text {delay }}=t_{R}-t_{D}=6.565 \times 10^{-4} \mathrm{~s} \\
\text { delay_in_samples } \\
=t_{\text {delay }} \times 192 \times 10^{3} \text { samples } / \mathrm{s} \\
=126
\end{gathered}
$$

A simple path loss calculation shows that the relative path loss of the skywave is approximately $36 \mathrm{~dB}$ or 0.0158 of the magnitude of the groundwave. These values were used to increase the realism of the artificial 2-ray signal.

\section{B.2.3 Long range two path}

The final step of this stage of the project was to transmit at full power from the dipole antenna and observe the reflected skywave. The signal correlation is shown in Figure B.3a. There is a spike in the impulse response for every sweep, spaced by the sweep duration. In this instance, the sweep was $0.5 \mathrm{~s}$ long. The details begin to emerge when those peaks are zoomed in on (Figure B.3b).

The first, very thin peak is the groundwave which travelled directly from the transmitting antenna to the receiving antenna. It is later used as a reference between transmissions to compare the rest of the data. The later, wider peaks are the skywaves. This can be verified by calculating how far the radio wave had to travel before being received. Since the time at which the groundwave and the skywave were transmitted is the same, and the time between their receptions is recorded, this is relatively simple. From Figure B.3b, the time between receptions is $0.00195 \mathrm{~s}$ and assuming that the radio wave travelled at $300,000 \frac{\mathrm{km}}{\mathrm{s}}$, it can be calculated that the skywave travelled $585 \mathrm{~km}$. This is consistent with the known height of the ionosphere [5].

Another data visualization was made by taking all of the correlation peaks from a single session (a session being the time in which a transmission happens and the receivers are recording) and overlaying them by using the groundwave as a reference. 


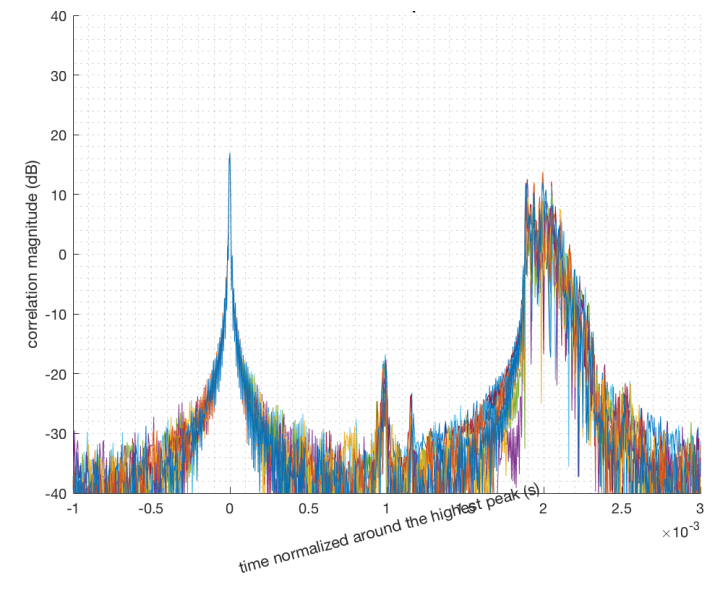

(a) All correlation peaks from a single session overlaid.

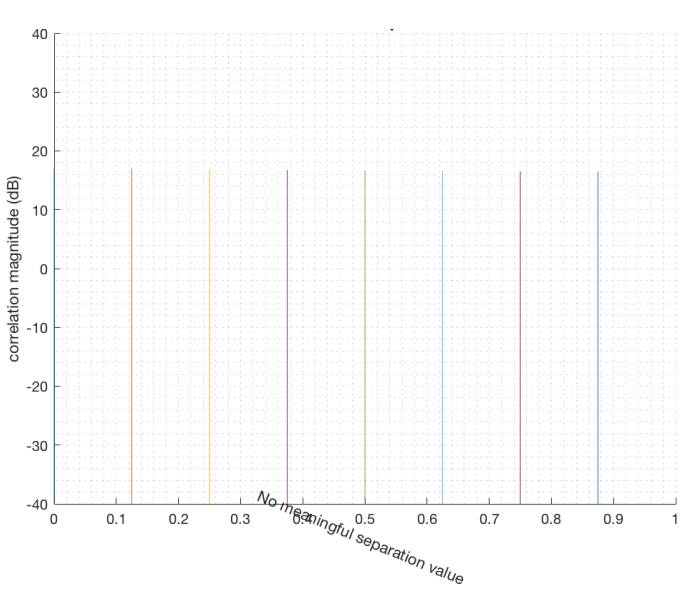

(b) Correlation peak amplitudes

FIGURE B.4: Overlaid correlation peaks.

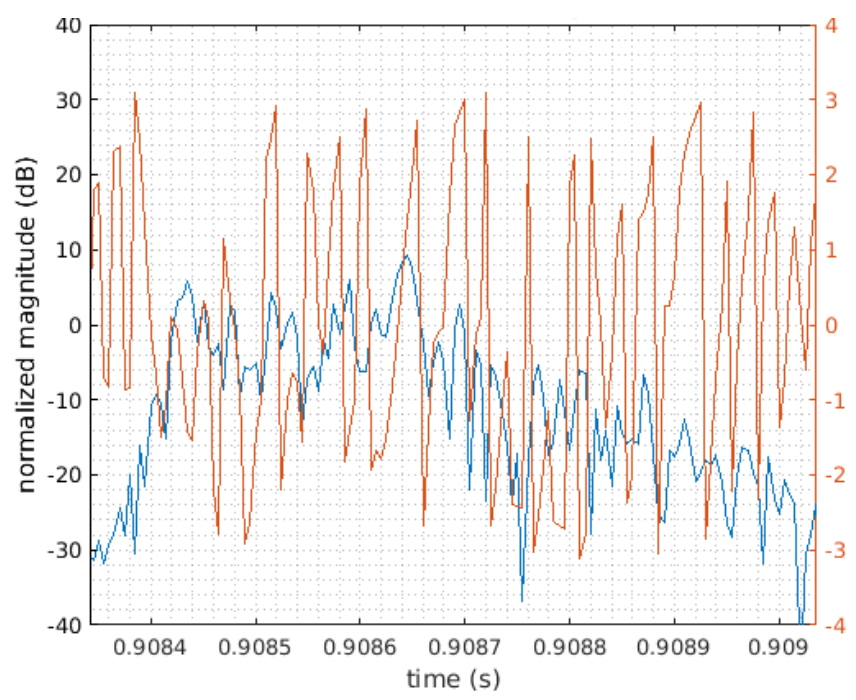

FIGURE B.5: Phase (orange, right y-axis) and magnitude (blue, left $\mathrm{y}$-axis) of the correlation zoomed in on a single peak.

The data surrounding each groundwave peak is normalized as if the groundwave peak occurred at time 0, then plotted in Figure B.4a.

This plot shows the consistency of the peaks throughout a session. This has implications regarding the frequencies of the ionospheric waves. When all of the spikes from a single session are seen side-by-side, their amplitudes can also be visually compared as in Figure B.4b. The phase information was also plotted throughout the correlation peak. By examining the phase of the signals received, information can be derived regarding the relative time taken for the signal to reach each antenna, and thereby the distance to the ionosphere can be determined. When looking at the skywave, (Figure B.5), the phase appears to be completely uncorrelated.

Looking at the groundwaves provide much more promising results. As can be seen in Figure B.6, for a given transmission, the phase at each correlation peak is non-constant, but when comparing the two receiving antennas, the phase difference across the 9 sweeps remains constant.

One final point of interest is that with each session, current weather data was 

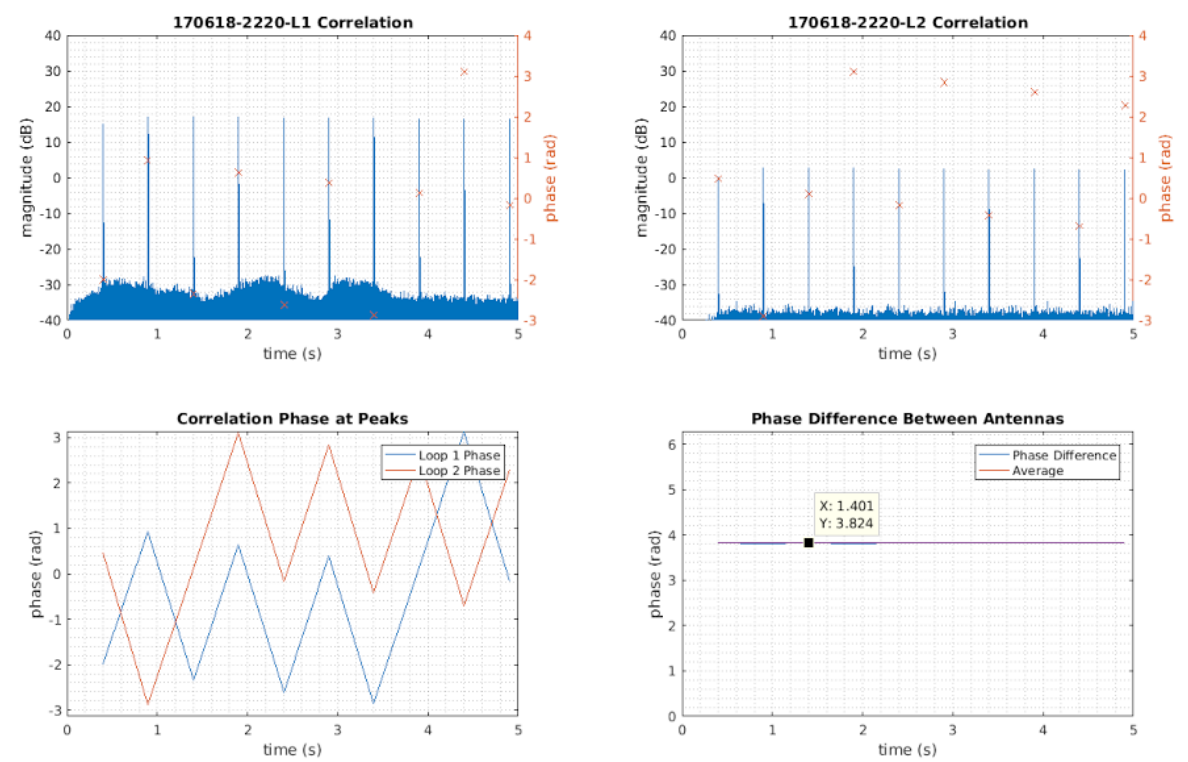

FIGURE B.6: Phase of the correlation over an entire transmission.

saved to a spreadsheet. This will allow for later analyzing trends between the collected atmospheric data and the weather patterns.

\section{B.3 Discussion and interpretation}

While the results presented in Section B.2 were compelling, there are some inconsistencies that bear examining.

\section{B.3.1 Skywave correlation}

As noted in Section B.2.3, while the phase information from the groundwave component of the correlation data was discernable, the phase of the skywave was indeterminate. It is possible that this was caused by the transmitted frequency sweep spreading the skywave. The literature supports that the depth a wave will penetrate into each layer of the ionosphere is a function of the frequency of the wave, which in turn supports the theory that the reception of the sweep will be temporally spaced out, resulting in an uncorrelated phase. One way to address this is to use a different high-autocorrelation signal. For example, the Barker codes can be transmitted on a single frequency, using binary amplitude shift keying (BASK), by flipping the phase by $180^{\circ}$ for each bit of information.

\section{B.3.2 Groundwave phase measurements}

The groundwave phase results are interesting but there is an inconsistency that requires discussion. Although the phase difference between the two antennas remains constant throughout a given transmission, it is non-constant between transmissions. The most likely cause of this anomaly is experimental error. For example, if the phase between the two USRPs was not properly locked then a random phase difference for each session would be entirely possible. 


\section{B.4 Conclusion}

In this appendix, the steps taken during a preliminary study of the ionosphere were detailed, and the viability of the technology to be used was discussed. Simulations were done which matched the eventually collected data. What can easily be attributable to ionospheric layer differences were verified by considering time delays between transmitted signals, and correlation peaks were overlaid to begin drawing what can be considered ionospheric waves. The instrumentation appears to not be consistently phased, which leads to questions about the validity of using the phase data. Overall, the experiment was a success and lays a strong foundation for the continuation of the project. 


\section{Appendix C}

\section{LWA proposed work}

As discussed in section 2.5, the LWA observatory in New Mexico is a high-quality facility operating with 256-phased antennas. They do offer observation time for which a proposal must be submitted and accepted. It was considered an inexpensive way to practice operating a large array while potentially collecting some higher-quality data than the feasibility study completed at DRAO (chapter 4).

A proposal was written by Dr. Peter Driessen and co-signed by Dr. Rodney Herring and Dr. Stephen Harrison. Data from this thesis were used in the proposal. As a result of the proposal, 16 observation hours were awarded to UViip for the 2019 year. This will require coordinating a research trip to New Mexico as well as building an off-site transmitter. 


\section{Appendix D}

\section{Miscellaneous work done in support of a Masters of Applied Science}

\section{D.1 GNU Radio implementation of Flex radio}

Mistic lab, run by Dr. Peter Driessen, is a research space focused on communications with special attention given to SDR technology. The lab is always making use of new software radios and trying to implement them in novel ways. This appendix outlines efforts to interface a powerful SDR (FlexRadio-6700) with an open-sourced software package (GNU Radio) over a communications network. All of the work done in this appendix was done collaboratively with Colter McQuay.

\section{D.1.1 Motivation}

There is a FlexRadio-6700 in the Mistic Laboratory but it was only accessible via FlexRadio's proprietary software (only available on Windows). It was found that they do have a publically available API to interface with their devices and so the idea was had to build a GNU Radio block that could communicate with the radio. This would allow the FlexRadio to be used by any computer running the free GNU Radio software, independent of the computers OS.

\section{D.1.2 Components}

\section{GNU Radio}

GNU Radio is an open-sourced software that supports a wide variety of software radios. It provides a framework for running these radios and doing signal processing on their inputs and outputs. It is primarily used as a user-friendly drag-and-drop flowgraph built from 'blocks'. These blocks range from signal generators, to datatype converters, to file-system integrations, to SDR interfaces.

\section{FlexRadio 6700}

The FlexRadio-6700 is an SDR built for amateur radio users. It is able to both receive and transmit on up to two antennas. On the receiver side, it is is able to take each antenna input and split it into eight panadapters (visual displays of both spectrum and waterfall). It makes use of a powerful ADC ( 250 MSPS) and DAC ( 500 MSPS) which allow it to generate so many panadapters. 


\section{GNU Radio}

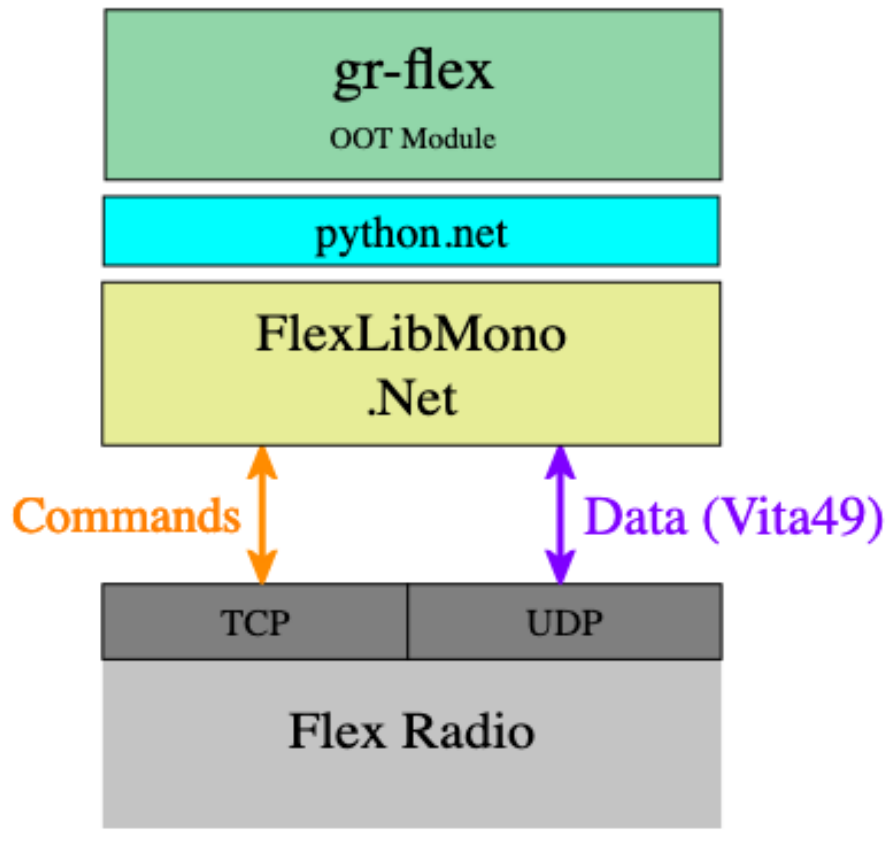

FIGURE D.1: The architecture of the GNU Radio interface with the Flex radio.

\section{D.1.3 Implementation}

The GNU Radio block was developed as an Out-of-Tree module (which means it must be independently maintained) and is build using Python2 (as GNU Radio blocks must be built in either $\mathrm{C}++$ or Python). The FlexRadio API is accessed via a user-developed library called FlexLibMono. In order to use this Mono package in Python, a package called pythonnet was used. An overview of this architecture and how the various components work together is shown in figure D.1.

The work done was primarily in the gr-flex layer of figure D.1.

\section{D.1.4 Results}

The block functions over either a ethernet connection and has been tested on both macOS and Linux systems. A screenshot of the block being used in a flowgraph is shown in figure D.2. The block has parameters which can be changed by the user real-time for center frequency, bandwidth, RX antenna, DAX IQ channel (there are four IQ channels available).

If multiple of the gr-flex blocks are added to a flowgraph, each will open a panadapter (up to a maximum of eight) on the FlexRadio.

\section{D.1.5 Existing issues}

On the proprietary FlexRadio software, there is a very smooth bandwidth adjustment available for each panadapter. So far the bandwidth adjustment in the block does not work. No serious effort has gone in to understanding why. 


\begin{tabular}{|c|c|c|}
\hline \begin{tabular}{l}
\multicolumn{1}{c}{ Options } \\
ID: top_block \\
Generate Options: WX GUI
\end{tabular} & $\begin{array}{l}\text { WX GUI Slider } \\
\text { ID: center_freq } \\
\text { Label: Frequency }\end{array}$ & $\begin{array}{l}\text { WX GUI Slider } \\
\text { ID: bandwidth } \\
\text { Label: Bandwidth }\end{array}$ \\
\hline $\begin{array}{c}\text { Variable } \\
\text { ID: samp_rate }\end{array}$ & $\begin{array}{l}\text { Minimum: } 500 \\
\text { Maximum: } 200 \mathrm{M} \\
\text { Converter: Float }\end{array}$ & $\begin{array}{l}\text { Minimum: } 15 \mathrm{k} \\
\text { Maximum: } 15 \mathrm{M} \\
\text { Converter: Float }\end{array}$ \\
\hline
\end{tabular}

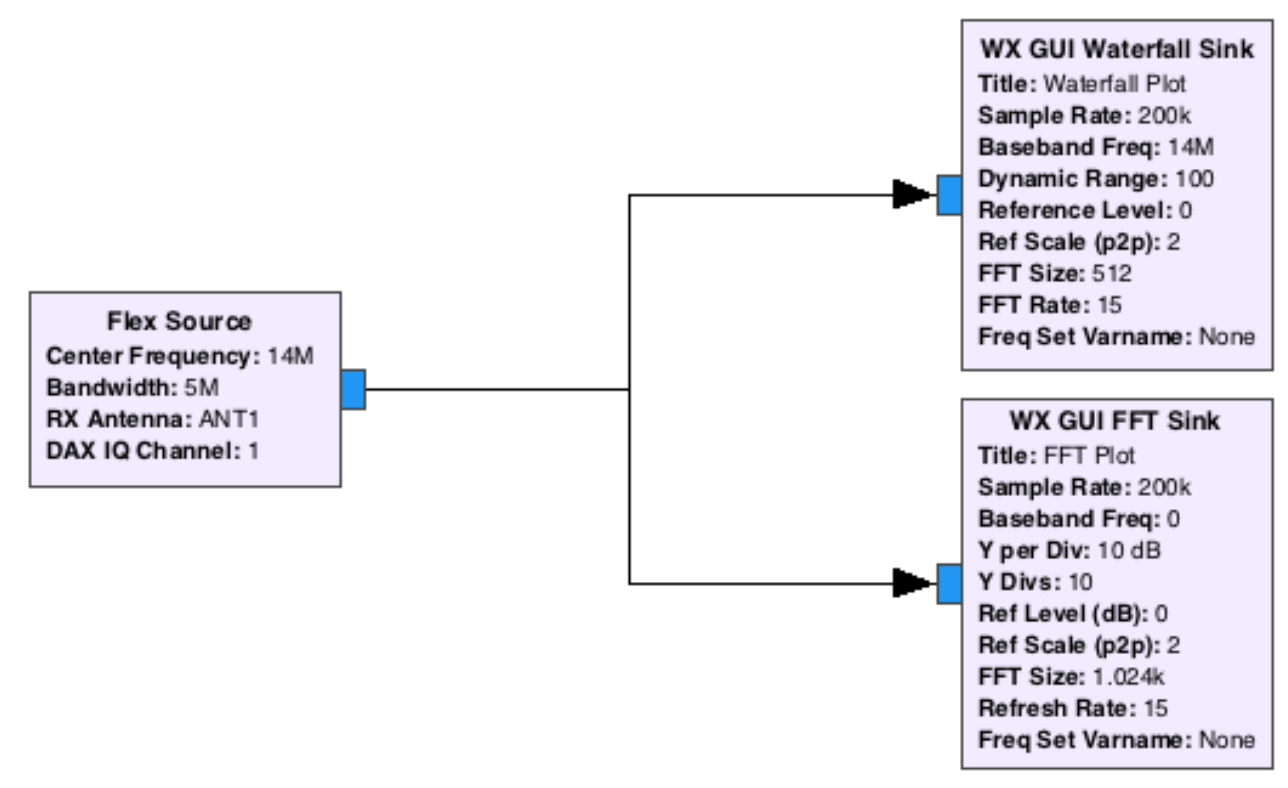

FIGURE D.2: The gr-flex block being used to visualize a waterfall and FFT in GNU Radio. 


\section{D.1.6 Release}

The source code and instructions on how to install the block are available on Github via Colter McQuay github.com/cjam/gr-flex. It will be moved to the Mistic Lab Github account github.com/misticlab once it has been finished. At that point it will also be announced to the FlexRadio community at large. It is an active community of amateur radio enthusiasts who would no doubt find value in this project. 


\section{Bibliography}

[1] H. Rishbeth, "F-region links with the lower atmosphere?", Journal of Atmospheric and Solar-Terrestrial Physics, vol. 68, no. 3-5, pp. 469-478, 2006, ISSN: 13646826. DOI: $10.1016 / j$. jastp. 2005.03.017.

[2] Y. I. Chen, J. Y. Liu, Y. B. Tsai, and C. S. Chen, "Statistical tests for preearthquake ionospheric anomaly", Terrestrial, Atmospheric and Oceanic Sciences, vol. 15, no. 3, pp. 385-396, 2004, ISSN: 10170839.

[3] U. of Victoria, Earthquake early-warning sensors installed off coast of British Columbia, Toronto, 2018. [Online]. Available: http : / / search . proquest . com. ezproxy. library. uvic.ca/docview/2123274401? accountid=14846.

[4] J. Chum, J.-Y. Liu, J. Laštovička, J. Fišer, Z. Mošna, J. Baše, and Y.-Y. Sun, “Ionospheric signatures of the April 25, 2015 Nepal earthquake and the relative role of compression and advection for Doppler sounding of infrasound in the ionosphere", Earth, Planets and Space, vol. 68, no. 1, p. 24, 2016, ISSN: 1880-5981. DOI: 10 .1186/s40623-016-0401-9. [Online]. Available: http: //www . earthplanets-space.com/content/68/1/24.

[5] Australian Government - IPS Radio and Space Services, "Introduction to HF radio propagation", Tech. Rep., 2010, pp. 1-25.

[6] H. Rishbeth, "How the thermospheric circulation affects the ionospheric F2-layer", Journal of Atmospheric and Solar-Terrestrial Physics, vol. 60, no. 14, pp. 1385-1402, 1998, ISSN: 13646826. DOI: 10.1016/S1364-6826 (98) 00062-5.

[7] M. Greenman, An Introduction to HF propagation and the Ionosphere, 2009. [Online]. Available: http://www.qsl.net/zl1bpu/IONO/iono101.htm.

[8] F5VZ, Ionosonde, 2003. [Online]. Available: https://en.wikipedia.org/wiki/ Ionosonde (visited on 08/01/2017).

[9] G. J.D.P.K.P. K. Schatten, "The solar activity cycle”, Space Science Reviews, vol. 53, pp. 325-357, 2015. DOI: 10 . 1007 / $978-1$ - 4939 - 2584 - 1. [Online]. Available: http://link. springer. com/10.1007/978-1-4939-2584-1.

[10] M. Thomas, First B.C. offshore earthquake early warning sensor up and running, 2016. [Online]. Available: http : / / www . cbc . ca / news / canada / british columbia/first-b-c-offshore-earthquake-early-warning-sensor - upand-running-1.3689186.

[11] V. Kim, V. Khegai, and P. Illich-Scitych, "On one possible ionospheric precursor of earthquakes", Physics of the solid earth, vol. 30, no. 3, pp. 223-226, 1994.

[12] E. Vance, "Earthquakes in the sky", Scientific American, vol. 319, no. 4, pp. 4449, 2018, ISSN: 00368733. [Online]. Available: http: //ezproxy . library .uvic . $\mathrm{ca} /$ login?url=http: //search. ebscohost. com/login. aspx?direct=true\{ $\backslash \&$ \} $\mathrm{db}=\mathrm{aph}\{\backslash \&\} \mathrm{AN}=131738388\{\backslash \&\}$ site=ehost - live $\{\backslash \&\}$ scope=site.

[13] S. Pulinets, K. Boyarchuk, and A. Karelin, "Conception and model of seismoionosphere-magnetosphere coupling", in Terra Scientific Publishing Co, 2002, pp. 351-361. 
[14] M. Hayakawa, Earthquake prediction with radio techniques, 11th ed. John Wiley \& Sons, Incorporated, 2015, ISBN: 9781118770405. [Online]. Available: https : //ebookcentral. proquest. com/lib/uvic/reader . action?docID $=4038546$.

[15] K. Heki, "Ionospheric electron enhancement preceding the 2011 Tohoku-Oki earthquake", Geophysical Research Letters, vol. 38, no. 17, pp. 1-5, 2011, ISSN: 00948276. DOI: 10.1029/2011GL047908.

[16] F. Masci, J. N. Thomas, F. Villani, J. A. Secan, and N. Rivera, "On the onset of ionospheric precursors 40 min before strong earthquakes", en, Journal of Geophysical Research A: Space Physics, vol. 120, no. 2, pp. 1383-1393, 2015, ISSN: 21699402. DOI: 10 . 1002 / 2014JA020822. [Online]. Available: http : / / doi . wiley.com/10.1002/2014JA020822.

[17] E Gherzi, "Ionosphere and weather", Nature, vol. 165, no. 4184, p. 38, 1950. DOI: $10.1038 / 165038 \mathrm{a} 0$.

[18] J. Lastovicka, Forcing of the ionosphere by waves from below, en, 2006. DOI: 10 . 1016 / j . jastp . 2005 . 01 . 018. [Online]. Available: http : / / linkinghub . elsevier.com/retrieve/pii/S1364682605002579.

[19] J. F. Helmboldt, T. E. Clarke, J. Craig, J. D. Dowell, S. W. Ellingson, J. M. Hartman, B. C. Hicks, N. E. Kassim, G. B. Taylor, and C. N. Wolfe, "Passive allSky imaging radar in the HF regime with WWV and the first station of the Long Wavelength Array", Radio Science, vol. 48, no. 5, pp. 491-512, 2013, ISSN: 00486604. DOI: 10.1002/rds . 20056.

[20] K. S. Obenberger, G. B. Taylor, J. M. Hartman, T. E. Clarke, J. Dowell, A. Dubois, D. Dubois, P. A. Henning, J. Lazio, S. Michalak, and F. K. Schinzel, "Monitoring the sky with the Prototype All-Sky Imager on the LWA1", Journal of Astronomical Instrumentation, vol. 04, no. 01n02, p. 1550 004, 2015, ISSN: 2251-1717. DOI: 10 . 1142/S225117171550004X. arXiv: arXiv: 1503 . 05150v1. [Online]. Available: http://www . worldscientific. com/doi/abs/10 .1142/ S225117171550004X.

[21] L. He and K. Heki, "Three-dimensional tomography of ionospheric anomalies immediately before the 2015 Illapel earthquake, central Chile", Journal of Geophysical Research: Space Physics, 2018, ISSN: 21699402. DOI: 10 . 1029 / 2017 JA024871.

[22] US Navy, Refraction in the ionosphere, 2016. [Online]. Available: http : / / www . tpub.com/neets/book10/40e.htm.

[23] N. Bruce, P. Kremler, G. Tanyer, P. Driessen, C. McQuay, R. Sharif, and R. Herring, "DRAO-UViip research collaboration proposal", University of Victoria, Victoria, Tech. Rep., 2017.

[24] N. Bruce, G. Tanyer, P. Driessen, and R. Herring, "Proposal for short-term data-collection effort hosted by DRAO", University of Victoria, Victoria, Tech. Rep., 2018, pp. 1-5.

[25] E\&I, 2100L power amplifier. [Online]. Available: http : / / rfamplifiers . eandiltd.com/Asset/2100LDataSheet (1).pdf.

[26] S. Harrison, UViip data collection at DRAO, Penticton, 2018.

[27] S Harrison, G Hovey, B Veidt, Z Ljusic, and T Burgess, A digital beamformer for the Advanced Focal Array Demonstrator (AFAD), 2018.

[28] DX Engineering, "Active receive antenna ARAH3-1P", Tech. Rep. 7, 2016, pp. 1-20. 
[29] Ettus Research, USRP N200 Software Defined Radio (SDR) - Ettus Research. [Online]. Available: https : / / www . ettus . com / product / details / UN200 - KIT (visited on 08/25/2017).

[30] DX Engineering, Active magnetic loop antennas DXE-RF-PRO-1B, 2017. [Online]. Available: https : //www .dxengineering.com/parts/dxe-rf-pro-1b (visited on $08 / 25 / 2017)$.

[31] C. C. Watterson, J. R. Juroshek, and W. D. Bensema, “Experimental confirmation of an HF channel model", IEEE Transactions on Communication Technology, vol. 18, no. 6, pp. 792-803, 1970, ISSN: 00189332. DOI: 10 .1109/TCOM . 1970 . 1090438. 